\author{
SUPPORTING INFORMATION \\ FOR AN ARTiCle In J. AM. CHEM. SOC.
}

SEPT. 28, 2016

\title{
Atmospheric Chemistry of Criegee Intermediates. Unimolecular Reactions and Reactions with Water
}

\author{
Bo Long ${ }^{a, b}$ Junwei Lucas Bao, ${ }^{b}$ and Donald G. Truhlar ${ }^{b} *$ \\ ${ }^{a}$ College of Information Engineering, Guizhou Minzu University, Guiyang, 550025, China \\ ${ }^{b}$ Department of Chemistry, Chemical Theory Center, and Supercomputing Institute, University of \\ Minnesota, Minneapolis, Minnesota 55455-0431, USA
}

TABLE OF CONTENTS

Table S1. Vibrational scale factors

Table S2. The critical geometrical parameters of $\mathrm{CH}_{2} \mathrm{OO}$

Table S3. The rotational constants of $\mathrm{CH}_{2} \mathrm{OO}, \mathrm{C} 1 \mathrm{~A}$, and $\mathrm{C} 1 \mathrm{~B}$ complexes

Table S4. The unscaled vibrational frequencies of $\mathrm{CH}_{2} \mathrm{OO}$

Table S5. The scaled vibrational frequencies of $\mathrm{CH}_{2} \mathrm{OO}$

S-6

Table S6. Frequencies and energies of precursor complexes C1A and C1B. S-7

Table S7. Classical barrier heights and energies of reaction. $\quad \mathrm{S}-8$

Table S8. The rotational constants of $s y n-\mathrm{CH}_{3} \mathrm{CHOO}$ and anti- $\mathrm{CH}_{3} \mathrm{CHOO}$ S-9

$\begin{array}{lr}\text { Table S9. Arrhenius activation energies } & \text { S-10 }\end{array}$

Table S10. The fractional contribution of the overall rate constant for every reaction $\quad$ S-11

Table S11. The pressure-dependent rate constants at differnet $\Delta E_{\text {down }} \quad$ S-12

Table S12. The tunneling transmission coefficient for reaction U2hs computed by $\begin{array}{ll}\text { various methods } & \mathrm{S}-13\end{array}$

$\begin{array}{ll}\text { Table S13. The transition pressure at the different temperature } & \text { S-14 }\end{array}$

$\begin{array}{lr}\text { Table S14. The concentrations of atmospheric species } & \text { S-15 }\end{array}$

$\begin{array}{lr}\text { Table S15. The concentrations of water monomer and dimer } & \text { S-16 }\end{array}$

$\begin{array}{lr}\text { Table S16. The rate constants at various temperature and pressure } & \text { S-17 }\end{array}$

$\begin{array}{lr}\text { Table S17. Cartesian coordinates of optimized structures. } & \text { S-18 }\end{array}$

$\begin{array}{lr}\text { Table S18. Absolute energies in hartrees } & \text { S-45 }\end{array}$

Figures S1-S15. Potential energy profiles along minimum energy paths calculated by MN15-L/MG3S $\quad$ S-50

$\begin{array}{lr}\text { Figures S16-18. Enthalpy profiles at } 0 \mathrm{~K} \text { for four reactions } & \text { S-58 }\end{array}$

Figures S19-24. Bimolecular and unimolecular rate constants as functions of $\begin{array}{ll}\text { temperature and pressure } & \text { S-60 }\end{array}$ 
Table S1. The scale factors for vibrational frequencies

\begin{tabular}{|c|c|}
\hline Methods & Scale Factor \\
\hline CCSD(T)-F12a/TZ-F12 $^{a}$ & 0.984 \\
\hline CCSD(T)-F12a/DZ-F12 & 0.983 \\
\hline QCISD/TZ & 0.975 \\
\hline MN15-L/MG3S & 0.977 \\
\hline MN15-L/maug-TZ & \\
\hline
\end{tabular}

${ }^{a}$ The text defines TZ as shorthand for cc-pVTZ, so TZ-F12 is shorthand for cc-pVTZ-F12.

${ }^{b}$ maug-cc-pV(T+d)Z is the same as maug-cc-pVTZ for molecules containing only $\mathrm{H}, \mathrm{C}$, and $\mathrm{O}$.

The text defines TZ as shorthand for cc-pVTZ, so maug-TZ denotes maug-cc-pTZ. 
Table S2. The critical bond lengths (in $\AA$ ) of $\mathrm{CH}_{2} \mathrm{OO}$ by various theoretical methods

\begin{tabular}{|c|c|c|}
\hline Methods & C-O & O-O \\
\hline CCSD(T)-F12a/TZ-F12 & 1.256 & 1.345 \\
\hline CCSD(T)-F12a/DZ-F12 & 1.256 & 1.345 \\
\hline QCISD/TZ & 1.266 & 1.354 \\
\hline MN15-L/MG3S & 1.260 & 1.342 \\
\hline MN15-L/maug-TZ & 1.260 & 1.345 \\
\hline M11-L/MG3S & 1.236 & 1.303 \\
\hline M11-L/maug-TZ & 1.238 & 1.308 \\
\hline M06-L/MG3S & 1.260 & 1.335 \\
\hline M06-L/maug-TZ & 1.260 & 1.338 \\
\hline
\end{tabular}


Table S3. The rotational constants (in $\mathrm{MHz}$ ) of $\mathrm{CH}_{2} \mathrm{OO}$ and complexes $\mathrm{B} 1 \mathrm{a}-\mathrm{C}$ and $\mathrm{B} 1 \mathrm{dh}-\mathrm{C}^{a}$

\begin{tabular}{|c|c|c|c|c|c|}
\hline \multicolumn{7}{|c|}{$\begin{array}{c}\text { CCSD(T)-F12a } \\
\text { /TZ-F12 }\end{array}$} & $\begin{array}{c}\text { CCSD(T)-F12a } \\
\text { /DZ-F12 }\end{array}$ & $\begin{array}{c}\text { QCISD } \\
\text { /aug-TZ }\end{array}$ & $\begin{array}{c}\text { QCISD } \\
\text { /TZ }\end{array}$ & $\begin{array}{c}\text { MN15-L } \\
\text { /MG3S }\end{array}$ \\
\hline \multicolumn{7}{|c|}{$\mathrm{CH}_{2} \mathrm{OO}$} \\
\hline$A$ & $78063.79(315)^{b}$ & $77777.40(29)$ & $75964.53(1784)$ & $76395.64(1353)$ & $80307.34(2558)$ \\
\hline$B$ & $12530.67(66)$ & $12518.06(53)$ & $12531.81(67)$ & $12563.56(99)$ & $12430.78(34)$ \\
\hline$C$ & $10797.48(76)$ & $10782.62(61)$ & $10757.20(36)$ & $10789.23(68)$ & $10764.54(43)$ \\
\hline$A$ & 11539.70 & 11548.45 & 11412.99 & 11330.39 & 11066.46 \\
\hline$B$ & 4737.31 & 4717.31 & 4728.55 & 4789.27 & 4671.19 \\
\hline$C$ & 3503.17 & 3489.09 & 3511.21 & 3557.24 & 3482.73 \\
\hline \multicolumn{7}{|c|}{$B 19-C$} \\
\hline$A$ & $12404.06(147)$ & $12391.39(135)$ & $12461.92(205)$ & $12389.04(132)$ & \\
\hline$B$ & $4313.63(91)$ & $4298.24(76)$ & $4212.79(10)$ & $4204.41(18)$ & \\
\hline$C$ & $3204.97(44)$ & $3197.27(37)$ & $3148.54(12)$ & $3155.31(5)$ & \\
\hline
\end{tabular}

$a$ The experimental rotational constants for $\mathrm{CH}_{2} \mathrm{OO}$ and the complex between $\mathrm{CH}_{2} \mathrm{OO}$ and $\mathrm{H}_{2} \mathrm{O}$ are

$$
\begin{aligned}
& A=77748.9 \\
& B=12465.03 \\
& C=10721.49
\end{aligned}
$$

for $\mathrm{CH}_{2} \mathrm{OO}$ and

$$
\begin{aligned}
& A=12256.729 \\
& B=4222.5955 \\
& C=3160.7314 .
\end{aligned}
$$

for B1dh-C.

${ }^{b}$ The values in parentheses are the differences between theory and experiment. 
Table S4. The unscaled vibrational frequencies (in $\mathrm{cm}^{-1}$ ) of $\mathrm{CH}_{2} \mathrm{OO}$

\begin{tabular}{|c|c|c|c|c|c|c|c|c|}
\hline Mode & Description & Sym. & Expt. $^{b}$ & VCI-5 $^{c}$ & $\begin{array}{c}\text { CCSD(T)- } \\
\text { F12a } \\
/ \text { TZ-F12 }\end{array}$ & $\begin{array}{c}\text { CCSD(T)- } \\
\text { F12a } \\
\text { /DZ-F12 }\end{array}$ & $\begin{array}{c}\text { QCISD } \\
\text { /TZ }\end{array}$ & $\begin{array}{c}\text { MN15-L } \\
\text { /MG3S }\end{array}$ \\
\hline$v_{1}$ & C-H antisym. str. & $a^{\prime}$ & - & 3151 & 3250 & 3305 & 3230 & 3191 \\
\hline$v_{2}$ & C-H sym. str. & $a^{\prime}$ & - & 3013 & 3090 & 3142 & 3082 & 3033 \\
\hline$v_{3}$ & $\mathrm{CH}_{2}$ scissor & $a^{\prime}$ & 1435 & 1434 & 1466 & 1489 & 1489 & 1514 \\
\hline$v_{4}$ & CO str./CH 2 scissor & $a^{\prime}$ & 1286 & 1285 & 1294 & 1309 & 1350 & 1376 \\
\hline$v_{5}$ & $\mathrm{CH}_{2}$ rock & $a^{\prime}$ & 1241 & 1212 & 1221 & 1240 & 1215 & 1223 \\
\hline$v_{6}$ & $\mathrm{OO}_{6}$ str. & $a^{\prime}$ & 908 & 927 & 937 & 952 & 972 & 946 \\
\hline$v_{7}$ & $\mathrm{COO}$ deform. & $a^{\prime}$ & - & 526 & 528 & 536 & 517 & 523 \\
\hline$v_{8}$ & $\mathrm{CH}_{2}$ wag & $a^{\prime \prime}$ & 848 & 859 & 857 & 862 & 902 & 915 \\
\hline$v_{9}$ & $\mathrm{CH}_{2}$ twist & $a^{\prime \prime}$ & - & 622 & 630 & 635 & 618 & 668 \\
\hline $\mathrm{MUE}^{a}$ & & & 0 & 12 & 19 & 27 & 52 & 58 \\
\hline
\end{tabular}

${ }^{a}$ Mean unsigned error averaged over five frequencies

${ }^{b}$ Su, Y. T.; Huang, Y. H.; Witek, H. A.; Lee, Y. P. Infrared Absorption Spectrum of the Simplest Criegee Intermediate $\mathrm{CH}_{2} \mathrm{OO}$. Science 2013, 340, 174-176.

${ }^{c}$ Li, J.; Carter, S.; Bowman, J. M.; Dawes, R.; Xie, D.; Guo, H., High-Level, First-Principles, Full-Dimensional Quantum Calculation of the Ro-vibrational Spectrum of the Simplest Criegee Intermediate $\left(\mathrm{CH}_{2} \mathrm{OO}\right)$. J. Phys. Chem. Lett. 2014, 5, 2364-2369. 
Table S5. The scaled vibrational frequencies (in $\mathrm{cm}^{-1}$ ) of $\mathrm{CH}_{2} \mathrm{OO}$

\begin{tabular}{|c|c|c|c|c|c|c|c|}
\hline Mode & Description & Sym. & Expt. & $\begin{array}{c}\text { CCSD(T) } \\
\text {-F12a } \\
/ \text { TZ-F12 }\end{array}$ & $\begin{array}{c}\text { CCSD(T) } \\
\text {-F12a } \\
\text { /DZ-F12 }\end{array}$ & $\begin{array}{c}\text { QCISD } \\
/ \text { TZ }\end{array}$ & $\begin{array}{c}\text { MN15-L } \\
\text { /MG3S }\end{array}$ \\
\hline$v_{1}$ & C-H antisym. str. & $a^{\prime}$ & - & 3198 & 3249 & 3149 & 3118 \\
\hline$v_{2}$ & C-H sym. str. & $a^{\prime}$ & - & 3041 & 3089 & 3005 & 2963 \\
\hline$v_{3}$ & $\mathrm{CH}_{2}$ scissor & $a^{\prime}$ & 1435 & 1443 & 1464 & 1452 & 1479 \\
\hline$v_{4}$ & $\mathrm{CO}$ str./CH scissor & $a^{\prime}$ & 1286 & 1273 & 1287 & 1316 & 1344 \\
\hline$v_{5}$ & $\mathrm{CH}_{2}$ rock & $a^{\prime}$ & 1241 & 1201 & 1219 & 1185 & 1195 \\
\hline$v_{6}$ & $\mathrm{OO}_{6}$ str. & $a^{\prime}$ & 908 & 922 & 936 & 948 & 924 \\
\hline$v_{7}$ & $\mathrm{COO}$ deform. & $a^{\prime}$ & - & 516 & 527 & 504 & 511 \\
\hline$v_{8}$ & $\mathrm{CH}_{2}$ wag & $a^{\prime \prime}$ & 848 & 843 & 847 & 879 & 894 \\
\hline$v_{9}$ & $\mathrm{CH}_{2}$ twist & $a^{\prime \prime}$ & - & 620 & 624 & 603 & 653 \\
\hline $\mathrm{MUE}^{a}$ & & & 0 & 16 & 16 & 35 & 42 \\
\hline
\end{tabular}

${ }^{a}$ Mean unsigned deviation from experiment averaged over five frequencies. 
Table S6. Frequencies (unscaled) and total energies of the two $\mathrm{CH}_{2} \mathrm{OO} \cdots \mathrm{H}_{2} \mathrm{O}$ complexes

\begin{tabular}{|c|c|c|c|c|c|c|c|}
\hline & Method & $\begin{array}{l}\text { Frequ } \\
\left(\mathrm{cm}^{-1}\right)\end{array}$ & ncies & & & $\begin{array}{l}E \\
\text { (hartrees) }\end{array}$ & $\begin{array}{l}\text { Relative } E \\
(\mathrm{kcal} / \mathrm{mol})\end{array}$ \\
\hline \multirow[t]{20}{*}{ B1a-C } & \multirow[t]{5}{*}{ QCISD/TZ } & 3942 & 3677 & 3317 & 3167 & \multirow[t]{5}{*}{$a=-265.627095$} & \multirow[t]{5}{*}{0.00} \\
\hline & & 1701 & 1545 & 1386 & 1262 & & \\
\hline & & 993 & 937 & 702 & 641 & & \\
\hline & & 531 & 387 & 213 & 186 & & \\
\hline & & 124 & 80 & & & & \\
\hline & \multirow[t]{5}{*}{ QCISD/aug-TZ } & 3923 & 3638 & 3305 & 3156 & \multirow[t]{5}{*}{$b=-265.652509$} & \multirow[t]{5}{*}{0.00} \\
\hline & & 1681 & 1539 & 1387 & 1258 & & \\
\hline & & 1004 & 918 & 666 & 636 & & \\
\hline & & 530 & 414 & 210 & 180 & & \\
\hline & & 133 & 77 & & & & \\
\hline & \multirow{5}{*}{$\begin{array}{l}\text { CCSD(T)-F12a } \\
/ \mathrm{DZ}-\mathrm{F} 12\end{array}$} & 3907 & 3591 & 3298 & 3140 & \multirow[t]{5}{*}{$c=-265.758548$} & \multirow[t]{5}{*}{0.00} \\
\hline & & 1675 & 1521 & 1364 & 1251 & & \\
\hline & & 958 & 879 & 671 & 642 & & \\
\hline & & 530 & 426 & 219 & 180 & & \\
\hline & & 127 & 57 & & & & \\
\hline & \multirow{5}{*}{$\begin{array}{l}\operatorname{CCSD}(\mathrm{T})-\mathrm{F} 12 \mathrm{a} \\
/ \mathrm{TZ}-\mathrm{F} 12\end{array}$} & 3906 & 3587 & 3296 & 3139 & \multirow[t]{5}{*}{$d=-265.793620$} & \multirow[t]{5}{*}{0.00} \\
\hline & & 1672 & 1522 & 1371 & 1252 & & \\
\hline & & 968 & 879 & 679 & 649 & & \\
\hline & & 532 & 428 & 221 & 188 & & \\
\hline & & 137 & 73 & & & & \\
\hline \multirow[t]{20}{*}{ B1dh-C } & \multirow[t]{5}{*}{ QCISD/TZ } & 3941 & 3684 & 3296 & 3134 & \multirow[t]{5}{*}{$e=-265.627256$} & \multirow{5}{*}{$\begin{array}{l}e-a=- \\
0.10\end{array}$} \\
\hline & & 1704 & 1545 & 1382 & 1290 & & \\
\hline & & 1015 & 956 & 697 & 669 & & \\
\hline & & 554 & 392 & 202 & 189 & & \\
\hline & & 146 & 69 & & & & \\
\hline & \multirow[t]{5}{*}{ QCISD/aug-TZ } & 3928 & 3653 & 3292 & 3138 & \multirow[t]{5}{*}{$f=-265.652309$} & \multirow{5}{*}{$\begin{array}{l}f-b \\
+0.13\end{array}$} \\
\hline & & 1679 & 1540 & 1384 & 1277 & & \\
\hline & & 1004 & 937 & 661 & 595 & & \\
\hline & & 551 & 471 & 200 & 151 & & \\
\hline & & 41 & $12 \mathrm{i}$ & & & & \\
\hline & \multirow{5}{*}{$\begin{array}{l}\text { CCSD(T)-F12a } \\
/ \mathrm{DZ}-\mathrm{F} 12\end{array}$} & 3911 & 3604 & 3287 & 3120 & \multirow[t]{5}{*}{$g=-265.758548$} & \multirow[b]{5}{*}{$g-c=0.00$} \\
\hline & & 1671 & 1521 & 1351 & 1269 & & \\
\hline & & 958 & 894 & 671 & 622 & & \\
\hline & & 552 & 465 & 212 & 158 & & \\
\hline & & & $7 \mathrm{i}$ & & & & \\
\hline & \multirow{5}{*}{$\begin{array}{l}\operatorname{CCSD}(\mathrm{T})-\mathrm{F} 12 \mathrm{a} \\
/ \mathrm{TZ}-\mathrm{F} 12\end{array}$} & 3910 & 3602 & 3284 & 3118 & \multirow[t]{5}{*}{$h=-265.793521$} & \\
\hline & & 1668 & 1522 & 1359 & 1269 & & \\
\hline & & 967 & 893 & 672 & 610 & & \\
\hline & & 552 & 470 & 213 & 158 & & $h-d$ \\
\hline & & 51 & 8 & & & & +0.06 \\
\hline
\end{tabular}


Table S7. The forward energy barriers $\left(\Delta E_{f}^{\ddagger}\right)$, reverse energy barriers $\left(\Delta E_{r}^{\ddagger}\right)$, and reaction energies $(\Delta E)$ of the reactions of Criegee intermediates with $\mathrm{H}_{2} \mathrm{O}$. Units: $\mathrm{kcal} / \mathrm{mol}$

\begin{tabular}{|c|c|c|c|c|c|c|c|}
\hline Method $^{a}$ & \multicolumn{2}{|c|}{$\Delta E_{f}^{\ddagger}$} & \multicolumn{2}{|c|}{$\Delta E_{r}^{\ddagger}$} & \multicolumn{2}{|c|}{$\Delta E$} & $\mathrm{MUE}^{b}$ \\
\hline \multicolumn{8}{|c|}{$\mathrm{CH}_{2} \mathrm{OO}+\mathrm{H}_{2} \mathrm{O} \longrightarrow \mathrm{CH}_{2}(\mathrm{OH}) \mathrm{OOH}$} \\
\hline & B1a-TS1 & B1a-TS2 & B1a-TS1 & B1a-TS2 & B1a-P1 & B1a-P2 & \\
\hline W3X-L//CCSD(T)-F12a/TZ-F12 & 0.49 & 1.45 & 46.91 & 47.66 & -46.42 & -46.19 & 0.00 \\
\hline W3X-L//CCSD(T)-F12a/DZ-F12 & 0.50 & 1.45 & 46.93 & 47.64 & -46.42 & -46.19 & 0.01 \\
\hline W3X-L//QCISD/TZ & 0.49 & 1.45 & 46.92 & 47.64 & -46.43 & -46.19 & 0.01 \\
\hline W3X//CCSD(T)-F12a/TZ-F12 & 0.47 & 1.39 & 46.92 & 47.60 & -46.45 & -46.21 & 0.03 \\
\hline $\mathrm{W} 3 \mathrm{X} / / \mathrm{QCISD} / \mathrm{TZ}$ & 0.44 & 1.37 & 46.91 & 47.59 & -46.47 & -46.22 & 0.05 \\
\hline M11-L/MG3S & 0.15 & 1.06 & 46.74 & 47.30 & -46.58 & -46.24 & 0.24 \\
\hline MN15-L/MG3S & 0.51 & 1.52 & 47.36 & 48.00 & -46.85 & -46.48 & 0.27 \\
\hline MN15-L/maug-TZ & -0.10 & 0.80 & 47.12 & 47.56 & -47.22 & -46.77 & 0.49 \\
\hline M11-L/maug-TZ & -0.01 & 0.72 & 46.34 & 46.69 & -46.36 & -45.97 & 0.51 \\
\hline W2X//CCSD(T)-F12a/TZ-F12 & -0.04 & 0.91 & 47.44 & 48.16 & -47.48 & -47.25 & 0.70 \\
\hline W2X//CCSD(T)-F12a/DZ-F12 & -0.04 & 0.90 & 47.44 & 48.16 & -47.48 & -47.25 & 0.71 \\
\hline $\mathrm{W} 2 \mathrm{X} / / \mathrm{QCISD} / \mathrm{TZ}$ & -0.12 & 0.83 & 47.44 & 48.15 & -47.56 & -47.32 & 0.75 \\
\hline M06-L/MG3S & 0.77 & 1.68 & 43.37 & 44.10 & -42.60 & -42.41 & 2.54 \\
\hline M06-L/maug-TZ & 0.30 & 1.10 & 42.93 & 43.44 & -42.63 & -42.35 & 2.73 \\
\hline \multicolumn{8}{|c|}{ syn $-\mathrm{CH}_{3} \mathrm{CHOO}+\mathrm{H}_{2} \mathrm{O} \longrightarrow \mathrm{HC}(\mathrm{OH}) \mathrm{CH}_{3} \mathrm{OOH}$} \\
\hline & B2a-TS1 & $\mathrm{B} 2 \mathrm{a}-\mathrm{TS} 2$ & B2a-TS1 & B2a-TS2 & B1a-P1 & B1a-P2 & \\
\hline W3X-L//QCISD/TZ & 5.15 & 6.51 & 45.02 & 45.94 & -39.87 & -39.43 & 0.00 \\
\hline W3X//QCISD/TZ & 5.09 & 6.41 & 45.04 & 45.93 & -39.95 & -39.51 & 0.06 \\
\hline W2X //QCISD/TZ & 4.66 & 6.01 & 45.51 & 46.42 & -40.85 & -40.41 & 0.65 \\
\hline MN15-L/ maug-TZ & 5.82 & 7.15 & 44.71 & 45.59 & -38.90 & -38.43 & 0.66 \\
\hline MN15-L/MG3S & 6.46 & 7.87 & 45.09 & 46.08 & -38.63 & -38.21 & 0.89 \\
\hline M11-L/MG3S & 5.53 & 6.95 & 43.95 & 44.75 & -38.42 & -37.80 & 1.03 \\
\hline M11-L/maug-TZ & 5.38 & 6.69 & 43.47 & 44.14 & -38.09 & -37.45 & 1.25 \\
\hline \multicolumn{8}{|c|}{ anti- $\mathrm{CH}_{3} \mathrm{CHOO}+\mathrm{H}_{2} \mathrm{O} \longrightarrow \mathrm{HC}(\mathrm{OH}) \mathrm{CH}_{3} \mathrm{OOH}$} \\
\hline & B3a-TS1 & B3a-TS2 & B3a-TS1 & B3a-TS2 & B3a-P1 & B3a-P2 & \\
\hline W3X-L//QCISD/TZ & -1.41 & -0.67 & 43.16 & 43.90 & -44.58 & -44.57 & 0.00 \\
\hline W3X//QCISD/TZ & -1.55 & -0.83 & 43.19 & 43.89 & -44.75 & -44.72 & 0.11 \\
\hline W2X//QCISD/TZ & -2.04 & -1.30 & 43.64 & 44.37 & -45.68 & -45.67 & 0.80 \\
\hline MN15-L/maug-TZ & -0.48 & 0.19 & 43.43 & 43.77 & -43.91 & -43.58 & 0.64 \\
\hline M11-L/maug-TZ & -1.02 & -0.54 & 41.85 & 42.27 & -42.87 & -42.81 & 1.16 \\
\hline
\end{tabular}

a As usual, "//" means "at a geometry optimized by". When no "//" is present, the method used for geometry is the same as the method used for energy.

${ }^{b}$ Mean unsigned error averaged over the six quantities in the previous columns 
Table S8. The rotational constants (in $\mathrm{MHz}$ ) of $s y n-\mathrm{CH}_{3} \mathrm{CHOO}$ and anti- $\mathrm{CH}_{3} \mathrm{CHOO}^{a}$

\begin{tabular}{|c|c|c|c|c|c|}
\hline & $\begin{array}{c}\text { CCSD(T)-F12a } \\
\text { /TZ-F12 }\end{array}$ & $\begin{array}{c}\text { CCSD(T)-F12a } \\
\text { /DZ-F12 }\end{array}$ & $\begin{array}{c}\text { QCISD } \\
\text { /TZ }\end{array}$ & $\begin{array}{c}\text { MN15-L } \\
\text { /maug-TZ }\end{array}$ & Experiment ${ }^{1}$ \\
\hline \multicolumn{5}{|c|}{ syn-CH ${ }_{3}$ CHOO } \\
\hline$A$ & $17601.90(15)$ & $17577.37(9)$ & $17548.42(38)$ & $17600.05(13)$ & 17586.5295 \\
\hline$B$ & $7195.36(62)$ & $7187.46(54)$ & $7170.67(37)$ & $7127.71(6)$ & 7133.4799 \\
\hline$C$ & $5269.85(41)$ & $5263.50(34)$ & $5251.85(23)$ & $5234.29(5)$ & 5229.1704 \\
\hline \multicolumn{7}{|c|}{ anti-CH3 ${ }_{3}$ CHOO } \\
\hline$A$ & $48828.27(334)$ & $48717.92(224)$ & $48691.68(197)$ & $48858.62(365)$ & 48494.0664 \\
\hline$B$ & $4450.49(16)$ & $4446.06(11)$ & $4445.75(11)$ & $4426.18(9)$ & 4434.7713 \\
\hline$C$ & $4184.26(18)$ & $4179.56(13)$ & $4179.03(12)$ & $4163.95(3)$ & 4166.6704 \\
\hline
\end{tabular}

$a$ The difference between theory and experiment is in parentheses.

1 Nakajima, M.; Endo, Y. Communication: Spectroscopic characterization of an alkyl substituted Criegee intermediate syn- $\mathrm{CH}_{3} \mathrm{CHOO}$ through pure rotational transitions. J. Chem. Phys. 2014, 140, article no. 011101. 
Table S9. Arrhenius activation energies $(\mathrm{kcal} / \mathrm{mol})$ for the high-pressure limit

\begin{tabular}{|c|c|c|c|c|c|c|}
\hline$T / \mathrm{K}$ & B1a & U1c & B2 & U2hs & B3a & B3c \\
\hline 190 & 2.10 & 16.89 & -1.46 & 2.91 & 0.06 & 14.19 \\
\hline 200 & 2.15 & 17.21 & -0.7 & 3.24 & 0.10 & 14.49 \\
\hline 210 & 2.20 & 17.48 & 0.08 & 3.58 & 0.14 & 14.74 \\
\hline 212.6 & 2.21 & 17.54 & 0.28 & 3.68 & 0.15 & 14.8 \\
\hline 215.5 & 2.23 & 17.61 & 0.5 & 3.78 & 0.16 & 14.86 \\
\hline 218.6 & 2.24 & 17.68 & 0.73 & 3.9 & 0.17 & 14.92 \\
\hline 220 & 2.25 & 17.7 & 0.84 & 3.95 & 0.17 & 14.94 \\
\hline 223.7 & 2.26 & 17.78 & 1.12 & 4.09 & 0.19 & 15.01 \\
\hline 229.7 & 2.29 & 17.89 & 1.55 & 4.32 & 0.21 & 15.1 \\
\hline 230 & 2.29 & 17.89 & 1.58 & 4.33 & 0.21 & 15.11 \\
\hline 235.1 & 2.31 & 17.97 & 1.94 & 4.54 & 0.23 & 15.18 \\
\hline 240 & 2.32 & 18.05 & 2.27 & 4.74 & 0.24 & 15.24 \\
\hline 249.9 & 2.36 & 18.17 & 2.91 & 5.16 & 0.28 & 15.34 \\
\hline 250 & 2.36 & 18.18 & 2.92 & 5.17 & 0.28 & 15.35 \\
\hline 259.3 & 2.39 & 18.28 & 3.47 & 5.58 & 0.31 & 15.42 \\
\hline 260 & 2.39 & 18.28 & 3.51 & 5.61 & 0.31 & 15.43 \\
\hline 266.1 & 2.41 & 18.34 & 3.84 & 5.89 & 0.33 & 15.46 \\
\hline 270 & 2.43 & 18.37 & 4.04 & 6.08 & 0.34 & 15.49 \\
\hline 271 & 2.43 & 18.38 & 4.09 & 6.13 & 0.35 & 15.49 \\
\hline 280 & 2.46 & 18.44 & 4.49 & 6.57 & 0.37 & 15.53 \\
\hline 283 & 2.47 & 18.46 & 4.62 & 6.72 & 0.38 & 15.54 \\
\hline 288.8 & 2.48 & 18.49 & 4.84 & 7.02 & 0.4 & 15.55 \\
\hline 290 & 2.49 & 18.5 & 4.88 & 7.08 & 0.4 & 15.56 \\
\hline 293 & 2.50 & 18.51 & 4.98 & 7.23 & 0.41 & 15.56 \\
\hline 295 & 2.50 & 18.52 & 5.04 & 7.34 & 0.42 & 15.57 \\
\hline 297 & 2.51 & 18.53 & 5.1 & 7.45 & 0.42 & 15.57 \\
\hline 298 & 2.51 & 18.54 & 5.13 & 7.5 & 0.43 & 15.57 \\
\hline 300 & 2.52 & 18.55 & 5.18 & 7.61 & 0.43 & 15.57 \\
\hline 310 & 2.55 & 18.58 & 5.41 & 8.16 & 0.46 & 15.58 \\
\hline 320 & 2.57 & 18.61 & 5.56 & 8.74 & 0.48 & 15.57 \\
\hline 330 & 2.60 & 18.63 & 5.63 & 9.33 & 0.51 & 15.56 \\
\hline 340 & 2.63 & 18.65 & 5.63 & 9.95 & 0.53 & 15.54 \\
\hline 350 & 2.65 & 18.66 & 5.55 & 10.59 & 0.56 & 15.52 \\
\hline
\end{tabular}


Table S10. The fractional contribution of individual transition state facets ${ }^{a}$ to the overall rate constant for the following reactions:

$$
\begin{array}{lr}
\mathrm{CH}_{2} \mathrm{OO}+\mathrm{H}_{2} \mathrm{O} & (\mathrm{B} 1 \mathrm{a}) \\
\text { syn }-\mathrm{CH}_{3} \mathrm{CHOO}+\mathrm{H}_{2} \mathrm{O} & (\mathrm{B} 2=\mathrm{B} 2 \mathrm{a}+\mathrm{B} 2 \mathrm{dh}) \\
\text { anti }-\mathrm{CH}_{3} \mathrm{CHOO}+\mathrm{H}_{2} \mathrm{O} & (\mathrm{B} 3 \mathrm{a})
\end{array}
$$

\begin{tabular}{|c|c|c|c|c|c|c|c|c|}
\hline & \multicolumn{3}{|c|}{ B1a $^{b}$} & \multicolumn{3}{c|}{$\mathrm{B}^{c}$} & \multicolumn{2}{c|}{ B3a $^{d}$} \\
\hline$T / \mathrm{K}$ & $F F_{\text {B1a-TS1 }}$ & $F F_{\text {B1a-TS2 }}$ & $F F_{\text {B2a-TS1 }}$ & $F F_{\text {B2a-TS2 }}$ & $F F_{\text {B2dh-TS1 }}$ & $F F_{\text {B2dh-TS2 }}$ & $F F_{\text {B3a-TS1 }}$ & $F F_{\text {B3a-TS2 }}$ \\
\hline 190 & 0.90 & 0.10 & 0.01 & 0.00 & 0.41 & 0.58 & 0.22 & 0.78 \\
\hline 200 & 0.89 & 0.11 & 0.02 & 0.00 & 0.41 & 0.57 & 0.23 & 0.77 \\
\hline 210 & 0.88 & 0.12 & 0.04 & 0.00 & 0.41 & 0.55 & 0.24 & 0.76 \\
\hline 220 & 0.87 & 0.13 & 0.06 & 0.00 & 0.40 & 0.53 & 0.25 & 0.75 \\
\hline 230 & 0.86 & 0.14 & 0.10 & 0.00 & 0.39 & 0.50 & 0.25 & 0.75 \\
\hline 240 & 0.85 & 0.15 & 0.15 & 0.01 & 0.37 & 0.47 & 0.26 & 0.74 \\
\hline 250 & 0.84 & 0.16 & 0.20 & 0.01 & 0.35 & 0.43 & 0.27 & 0.73 \\
\hline 260 & 0.84 & 0.16 & 0.26 & 0.02 & 0.33 & 0.39 & 0.28 & 0.72 \\
\hline 270 & 0.83 & 0.17 & 0.32 & 0.02 & 0.30 & 0.35 & 0.28 & 0.72 \\
\hline 280 & 0.82 & 0.18 & 0.38 & 0.03 & 0.28 & 0.32 & 0.29 & 0.71 \\
\hline 290 & 0.81 & 0.19 & 0.43 & 0.04 & 0.26 & 0.28 & 0.30 & 0.70 \\
\hline 297 & 0.81 & 0.19 & 0.46 & 0.04 & 0.24 & 0.26 & 0.30 & 0.70 \\
\hline 298 & 0.81 & 0.19 & 0.46 & 0.04 & 0.24 & 0.26 & 0.30 & 0.70 \\
\hline 300 & 0.81 & 0.19 & 0.47 & 0.04 & 0.23 & 0.25 & 0.30 & 0.70 \\
\hline 310 & 0.80 & 0.20 & 0.51 & 0.05 & 0.22 & 0.23 & 0.31 & 0.69 \\
\hline 320 & 0.79 & 0.21 & 0.54 & 0.06 & 0.20 & 0.21 & 0.31 & 0.69 \\
\hline 330 & 0.79 & 0.21 & 0.56 & 0.06 & 0.19 & 0.19 & 0.32 & 0.68 \\
\hline 340 & 0.78 & 0.22 & 0.58 & 0.07 & 0.18 & 0.18 & 0.32 & 0.68 \\
\hline 350 & 0.77 & 0.23 & 0.60 & 0.07 & 0.17 & 0.16 & 0.33 & 0.67 \\
\hline
\end{tabular}

${ }^{a}$ The transition state is multi-faceted with each facet corresponding to a different saddle point (saddle points are also called transition structures).

$$
\begin{aligned}
& { }^{b} F_{\mathrm{B} 1 \mathrm{a}-\mathrm{TS} 1}=\frac{k_{B 1 a-T S 1}}{k_{B 1 a-T S 1}+k_{B 1 a-T S 2}} \text { and } F_{\mathrm{B} 1 \mathrm{a}-\mathrm{TS} 2}=\frac{k_{B 1 a-T S 2}}{k_{B 1 a-T S 1}+k_{B 1 a-T S 2}} \\
& { }^{c} F_{\mathrm{B} 2 \mathrm{a}-\mathrm{TS} 1}=\frac{k_{B 2 a-T S 1}}{k_{B 2 a-T S 1}+k_{B 2 a-T S 2}+k_{B 2 d h-T S 1+k_{B 2 d h-T S 2}}}, \\
& F_{\mathrm{B} 2 \mathrm{a}-\mathrm{TS} 2}=\frac{k_{B 2 a-T S 2}}{k_{B 2 a-T S 1}+k_{B 2 a-T S 2}+k_{B 2 d h-T S 1+k_{B 2 d h-T S 2}}} \\
& F_{\mathrm{B} 2 \mathrm{dh}-\mathrm{TS} 1}=\frac{k_{B 2 d h-T S 1}}{k_{B 2 a-T S 1}+k_{B 2 a-T S 2}+k_{B 2 d h-T S 1+k_{B 2 d h-T S 2}}}, \\
& \text { and } F_{\mathrm{B} 2 \mathrm{dh}-\mathrm{TS} 2}=\frac{k_{B 2 d h-T S 2}}{k_{B 2 a-T S 1}+k_{B 2 a-T S 2}+k_{B 2 d h-T S 1+k_{B 2 d h-T S 2}}} \\
& { }^{d} F_{\mathrm{B} 3 \mathrm{a}-\mathrm{TS} 1}=\frac{k_{B 3 a-T S 1}}{k_{B 3 a-T S 1}+k_{B 3 a-T S 2}} \text { and } F_{\mathrm{B} 3 \mathrm{a}-\mathrm{TS} 2}=\frac{k_{B 3 a-T S 2}}{k_{B 3 a-T S 1}+k_{B 3 a-T S 2}}
\end{aligned}
$$


Table S11. The pressure-dependent rate constants of the unimolecular reaction of $\mathrm{CH}_{2} \mathrm{OO}$ at $298 \mathrm{~K}$ as functions of pressure and the average energy transfer per collision $\left(\Delta E_{\text {down }}\right)$

\begin{tabular}{|c|c|c|c|}
\hline$P / \mathrm{mbar}$ & $\Delta E_{\text {down }}=300 \mathrm{~cm}^{-1}$ & $\Delta E_{\text {down }}=400 \mathrm{~cm}^{-1}$ & $\Delta E_{\text {down }}=500 \mathrm{~cm}^{-1}$ \\
\hline $1.00 \mathrm{E}+6$ & $3.07 \mathrm{E}-1$ & $3.07 \mathrm{E}-1$ & $3.07 \mathrm{E}-1$ \\
\hline $1.00 \mathrm{E}+5$ & $3.00 \mathrm{E}-1$ & $3.02 \mathrm{E}-1$ & $3.03 \mathrm{E}-1$ \\
\hline $1.01 \mathrm{E}+3$ & $9.67 \mathrm{E}-2$ & $1.13 \mathrm{E}-1$ & $1.24 \mathrm{E}-1$ \\
\hline $9.80 \mathrm{E}+2$ & $9.45 \mathrm{E}-2$ & $1.10 \mathrm{E}-1$ & $1.22 \mathrm{E}-1$ \\
\hline $5.42 \mathrm{E}+2$ & $6.07 \mathrm{E}-2$ & $7.29 \mathrm{E}-2$ & $8.18 \mathrm{E}-2$ \\
\hline $2.69 \mathrm{E}+2$ & $3.35 \mathrm{E}-2$ & $4.12 \mathrm{E}-2$ & $4.70 \mathrm{E}-2$ \\
\hline $1.33 \mathrm{E}+2$ & $1.76 \mathrm{E}-2$ & $2.19 \mathrm{E}-2$ & $2.53 \mathrm{E}-2$ \\
\hline $1.22 \mathrm{E}+2$ & $1.62 \mathrm{E}-2$ & $2.02 \mathrm{E}-2$ & $2.33 \mathrm{E}-2$ \\
\hline $5.50 \mathrm{E}+1$ & $7.51 \mathrm{E}-3$ & $9.44 \mathrm{E}-3$ & $1.10 \mathrm{E}-2$ \\
\hline $4.00 \mathrm{E}+1$ & $5.50 \mathrm{E}-3$ & $6.92 \mathrm{E}-3$ & $8.05 \mathrm{E}-3$ \\
\hline $2.50 \mathrm{E}+1$ & $3.46 \mathrm{E}-3$ & $4.36 \mathrm{E}-3$ & $5.08 \mathrm{E}-3$ \\
\hline $1.15 \mathrm{E}+1$ & $1.60 \mathrm{E}-3$ & $2.02 \mathrm{E}-3$ & $2.36 \mathrm{E}-3$ \\
\hline $9.33 \mathrm{E}+1$ & $1.30 \mathrm{E}-3$ & $1.64 \mathrm{E}-3$ & $1.92 \mathrm{E}-3$ \\
\hline $6.80 \mathrm{E}+1$ & $9.49 \mathrm{E}-4$ & $1.20 \mathrm{E}-3$ & $1.40 \mathrm{E}-3$ \\
\hline $5.40 \mathrm{E}+1$ & $7.54 \mathrm{E}-4$ & $9.53 \mathrm{E}-4$ & $1.11 \mathrm{E}-3$ \\
\hline $2.70 \mathrm{E}+1$ & $3.78 \mathrm{E}-4$ & $4.77 \mathrm{E}-4$ & $5.57 \mathrm{E}-4$ \\
\hline $1.40 \mathrm{E}+1$ & $1.96 \mathrm{E}-4$ & $2.48 \mathrm{E}-4$ & $2.89 \mathrm{E}-4$ \\
\hline $7.30 \mathrm{E}-1$ & $1.02 \mathrm{E}-4$ & $1.29 \mathrm{E}-4$ & $1.51 \mathrm{E}-4$ \\
\hline
\end{tabular}


Table S12. Tunneling transmission coefficients (unitless) and rate constants $\left(\mathrm{s}^{-1}\right)$ for reaction through transition structure U2hs-TS of Figure 2 as computed by the Eckart, ${ }^{a} \mathrm{ZCT}$, and SCT approximations

\begin{tabular}{|c|c|c|c|c|c|c|c|}
\hline & \multicolumn{2}{|c|}{ ref. $78^{b}$} & \multicolumn{2}{|c|}{$\mathrm{W} 3 \mathrm{X}-\mathrm{L}^{c}$} & \multicolumn{3}{|c|}{ MN15-L/maug-TZ ${ }^{d}$} \\
\hline$T / \mathrm{K}$ & $\kappa^{\text {Eckart }}$ & $k^{\mathrm{TST} / \text { Eckart }}$ & $\kappa^{\text {Eckart }}$ & $k^{\mathrm{TST} / \mathrm{Eckart}}$ & $\kappa^{\text {Eckart }}$ & $\kappa^{\mathrm{ZCT}}$ & $\kappa^{\mathrm{SCT}}$ \\
\hline 190 & $1.11 \mathrm{E}+7$ & 1.24 & $8.30 \mathrm{E}+6$ & 1.14 & $1.06 \mathrm{E}+6$ & $1.68 \mathrm{E}+5$ & $2.62 \mathrm{E}+7$ \\
\hline 200 & $1.75 \mathrm{E}+6$ & 1.91 & $1.33 \mathrm{E}+6$ & 1.77 & $1.95 \mathrm{E}+5$ & $3.79 \mathrm{E}+4$ & $4.13 E+6$ \\
\hline 210 & $3.45 E+5$ & 2.94 & $2.65 E+5$ & 2.75 & $4.45 E+4$ & $1.05 E+4$ & $8.06 E+5$ \\
\hline 220 & $8.26 \mathrm{E}+4$ & 4.58 & $6.45 E+4$ & 4.33 & $1.23 E+4$ & $3.49 \mathrm{E}+3$ & $1.89 \mathrm{E}+5$ \\
\hline 230 & $2.35 \mathrm{E}+4$ & 7.20 & $1.86 \mathrm{E}+4$ & 6.87 & $4.02 \mathrm{E}+3$ & $1.35 \mathrm{E}+3$ & $5.19 E+4$ \\
\hline 240 & $7.74 \mathrm{E}+3$ & $1.14 \mathrm{E}+1$ & $6.23 E+3$ & $1.10 \mathrm{E}+1$ & $1.52 \mathrm{E}+3$ & $5.91 \mathrm{E}+2$ & $1.65 E+4$ \\
\hline 250 & $2.92 \mathrm{E}+3$ & $1.82 \mathrm{E}+1$ & $2.39 E+3$ & $1.77 E+1$ & $6.54 \mathrm{E}+2$ & $2.90 \mathrm{E}+2$ & $5.91 E+3$ \\
\hline 260 & $1.24 \mathrm{E}+3$ & $2.92 \mathrm{E}+1$ & $1.03 E+3$ & $2.87 E+1$ & $3.15 \mathrm{E}+2$ & $1.57 \mathrm{E}+2$ & $2.37 E+3$ \\
\hline 270 & $5.86 \mathrm{E}+2$ & $4.72 \mathrm{E}+1$ & $4.92 E+2$ & $4.68 E+1$ & $1.68 \mathrm{E}+2$ & $9.26 \mathrm{E}+1$ & $1.06 \mathrm{E}+3$ \\
\hline 280 & $3.04 \mathrm{E}+2$ & $7.68 \mathrm{E}+1$ & $2.59 \mathrm{E}+2$ & $7.71 \mathrm{E}+1$ & $9.77 \mathrm{E}+1$ & $5.86 \mathrm{E}+1$ & $5.14 E+2$ \\
\hline 290 & $1.71 E+2$ & $1.25 E+2$ & $1.48 \mathrm{E}+2$ & $1.27 E+2$ & $6.13 E+1$ & $3.95 \mathrm{E}+1$ & $2.72 \mathrm{E}+2$ \\
\hline 297 & $1.20 \mathrm{E}+2$ & $1.77 \mathrm{E}+2$ & $1.04 \mathrm{E}+2$ & $1.80 \mathrm{E}+2$ & $4.59 \mathrm{E}+1$ & $3.09 \mathrm{E}+1$ & $1.82 \mathrm{E}+2$ \\
\hline 298 & $1.14 \mathrm{E}+2$ & $1.86 \mathrm{E}+2$ & $9.94 \mathrm{E}+1$ & $1.90 \mathrm{E}+2$ & $4.42 \mathrm{E}+1$ & $2.99 \mathrm{E}+1$ & $1.73 E+2$ \\
\hline 300 & $1.04 \mathrm{E}+2$ & $2.06 \mathrm{E}+2$ & $9.06 \mathrm{E}+1$ & $2.10 \mathrm{E}+2$ & $4.10 \mathrm{E}+1$ & $2.80 \mathrm{E}+1$ & $1.55 \mathrm{E}+2$ \\
\hline 310 & $6.69 \mathrm{E}+1$ & $3.36 \mathrm{E}+2$ & $5.92 \mathrm{E}+1$ & $3.46 \mathrm{E}+2$ & $2.89 \mathrm{E}+1$ & $2.08 \mathrm{E}+1$ & $9.48 \mathrm{E}+1$ \\
\hline 320 & $4.57 \mathrm{E}+1$ & $5.49 \mathrm{E}+2$ & $4.09 \mathrm{E}+1$ & $5.70 \mathrm{E}+2$ & $2.14 \mathrm{E}+1$ & $1.61 \mathrm{E}+1$ & $6.14 \mathrm{E}+1$ \\
\hline 330 & $3.27 \mathrm{E}+1$ & $8.91 E+2$ & $2.96 \mathrm{E}+1$ & $9.33 \mathrm{E}+2$ & $1.65 \mathrm{E}+1$ & $1.28 \mathrm{E}+1$ & $4.20 \mathrm{E}+1$ \\
\hline 340 & $2.44 \mathrm{E}+1$ & $1.44 \mathrm{E}+3$ & $2.23 \mathrm{E}+1$ & $1.51 \mathrm{E}+3$ & $1.31 \mathrm{E}+1$ & $1.05 \mathrm{E}+1$ & $3.01 \mathrm{E}+1$ \\
\hline 350 & $1.89 \mathrm{E}+1$ & $2.31 \mathrm{E}+3$ & $1.74 \mathrm{E}+1$ & $2.44 \mathrm{E}+3$ & $1.08 \mathrm{E}+1$ & 8.79 & $2.25 \mathrm{E}+1$ \\
\hline
\end{tabular}

$a$ In each case the Eckart parameters were chosen such that the potential energy of reaction equals the enthalpy of reaction at $0 \mathrm{~K}$, the barrier height equals the enthalpy of activation at $0 \mathrm{~K}$, and the second derivative of the potential at the barrier top, when combined with the reduced mass, reproduces the imaginary frequency at the saddle point. The reduced mass $\mu$ was taken to be $1.008 \mathrm{amu}$. The Eckart tunneling is calculated by eqs 2 and A7 in a previous paper. ${ }^{2}$

${ }^{b}$ For Eckart tunneling, the parameters we used are: $\Delta H_{0}^{\circ}=-18.10 \mathrm{kcal} / \mathrm{mol}, \Delta H_{0}^{\ddagger \text { o }}=17.05 \mathrm{kcal} / \mathrm{mol}$, $v^{\ddagger}=1696 \mathrm{i} \mathrm{cm}^{-1}$, which are obtained from Ref. 78. From these, we calculated $\alpha=2.41 \mathrm{bohr}^{-1}$, where $\alpha$ is an Eckart parameter defined in ref. 2.

$c$ For Eckart tunneling, the parameters we used are: $\Delta H_{0}^{\mathrm{o}}=-18.25 \mathrm{kcal} / \mathrm{mol}, \Delta H_{0}^{\ddagger .,}=17.01 \mathrm{kcal} / \mathrm{mol}$, $v^{\ddagger}=1677 \mathrm{i} \mathrm{cm}^{-1}, \alpha=2.38 \mathrm{bohr}^{-1}$, which are from our own best values that are obtained by W3X-L//CCSD(T)-F12a/DZ-F12 with the CCSD(T)-F12a/DZ-F12 scale factor of Table S1.

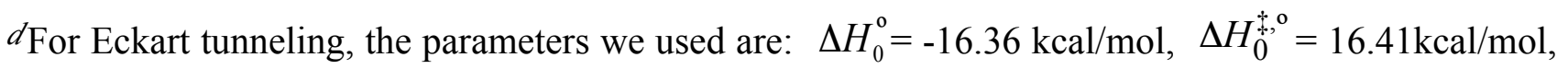
$v^{\ddagger}=1566 \mathrm{i} \mathrm{cm}^{-1}, \alpha=2.28 \mathrm{bohr}^{-1}$ from our own best DFT values that are obtained by MN15-L/maug-TZ with the MN15-L/maug-TZ scale factor of Table S1.

${ }^{2}$ Garrett, B. C.; Truhlar, D. G., Semiclassical tunneling calculations. J. Phys. Chem. 1979, 83, 2921-2926. 
Table S13. The transition pressure at various temperatures

\begin{tabular}{|l|l|l|}
\hline Reaction & $T / \mathrm{K}$ & $P_{1 / 2}(\mathrm{bar})$ \\
\hline \multirow{4}{*}{ U1c } & 190 & 0.07 \\
\cline { 2 - 3 } & 240 & 1.27 \\
\cline { 2 - 3 } & 298 & 11.65 \\
\cline { 2 - 3 } & 350 & 16.30 \\
\hline \multirow{4}{*}{ U2hs } & 190 & very low \\
\cline { 2 - 3 } & 240 & very low \\
\cline { 2 - 3 } & 298 & very low \\
\cline { 2 - 3 } & 350 & 0.004 \\
\hline \multirow{4}{*}{ U3c } & 190 & 0.007 \\
\cline { 2 - 3 } & 240 & 0.082 \\
\cline { 2 - 3 } & 298 & 0.199 \\
\cline { 2 - 3 } & 350 & 0.279 \\
\hline
\end{tabular}


Table S14. Water and ozone concentration and the concentration of water dimers at various altitudes ${ }^{a}$ (concentration unit: molecules $/ \mathrm{cm}^{3}$ )

\begin{tabular}{|c|c|c|c|c|c|c|c|}
\hline $\begin{array}{c}\text { Altitude } \\
\mathrm{km}\end{array}$ & $\begin{array}{c}T^{a} \\
\mathrm{~K}\end{array}$ & $\begin{array}{c}P \\
\mathrm{mbar}\end{array}$ & {$\left[\mathrm{H}_{2} \mathrm{O}\right]^{a}$} & {$\left[\mathrm{O}_{3}\right]^{a}$} & $K_{e q}{ }^{b}$ & {$\left[\left(\mathrm{H}_{2} \mathrm{O}\right)_{2}\right]$} & $\begin{array}{c}{\left[\left(\mathrm{H}_{2} \mathrm{O}\right)_{2}\right] /} \\
{\left[\mathrm{H}_{2} \mathrm{O}\right]}\end{array}$ \\
\hline 0 & 288.8 & 1013.25 & $3.5 \times 10^{17}$ & $7.5 \times 10^{11}$ & $2.44 \times 10^{-21}$ & $2.99 \mathrm{E}+14$ & $8.5 \mathrm{E}-04$ \\
\hline 5 & 259.3 & 542 & $1.5 \times 10^{16}$ & $7.7 \times 10^{11}$ & $4.62 \times 10^{-21}$ & $1.04 \mathrm{E}+12$ & $6.9 \mathrm{E}-05$ \\
\hline 10 & 229.7 & 269 & $3.1 \times 10^{14}$ & $7.6 \times 10^{11}$ & $1.05 \times 10^{-20}$ & $1.01 \mathrm{E}+09$ & $3.3 \mathrm{E}-06$ \\
\hline 15 & 212.6 & 122 & $1.2 \times 10^{13}$ & $2.2 \times 10^{12}$ & $1.90 \times 10^{-20}$ & $2.74 \mathrm{E}+06$ & $2.3 \mathrm{E}-07$ \\
\hline 20 & 215.5 & 55 & $5.9 \times 10^{12}$ & $4.9 \times 10^{12}$ & $1.71 \times 10^{-20}$ & $5.95 \mathrm{E}+05$ & $1.0 \mathrm{E}-07$ \\
\hline 25 & 218.6 & 25 & $3.2 \times 10^{12}$ & $4.9 \times 10^{12}$ & $1.53 \times 10^{-20}$ & $1.57 \mathrm{E}+05$ & $4.9 \mathrm{E}-08$ \\
\hline 30 & 223.7 & 11.5 & $1.6 \times 10^{12}$ & $3.1 \times 10^{12}$ & $1.29 \times 10^{-20}$ & $3.30 \mathrm{E}+04$ & $2.1 \mathrm{E}-08$ \\
\hline 35 & 235.1 & 5.4 & $8.2 \times 10^{11}$ & $1.4 \times 10^{12}$ & $8.93 \times 10^{-21}$ & $6.00 \mathrm{E}+03$ & $7.3 \mathrm{E}-09$ \\
\hline 40 & 249.9 & 2.7 & $4.0 \times 10^{11}$ & $3.9 \times 10^{11}$ & $5.87 \times 10^{-21}$ & $9.39 \mathrm{E}+02$ & $2.3 \mathrm{E}-09$ \\
\hline 45 & 266.1 & 1.4 & $2.1 \times 10^{11}$ & $1.3 \times 10^{11}$ & $3.95 \times 10^{-21}$ & $1.74 \mathrm{E}+02$ & $8.3 \mathrm{E}-10$ \\
\hline 50 & 271.0 & 0.73 & $1.1 \times 10^{11}$ & $4.2 \times 10^{10}$ & $3.49 \times 10^{-21}$ & $4.22 \mathrm{E}+01$ & $3.8 \mathrm{E}-10$ \\
\hline
\end{tabular}

${ }^{a}$ Data is from Brasseur, G.; Solomon, S., Aeronomy of the Middle Atmosphere: Chemistry and Physics of the Stratosphere and Mesosphere, Springer Netherlands: Dordrecht, 1986; pp. 441-442. This data is also used in Frost, G. J.; Vaida, V., Atmospheric implications of the photolysis of the ozone-water weakly bound complex. J.Geophys. Res., [Atmos.] 1995, 100 (D9), 18803-18809.

${ }^{b} K_{e q}$ is the equilibrium constant (in $\mathrm{cm}^{3} /$ molecule) for the association of two water monomers to make a dimer; it is calculated by cubic spline data interpolation of Table S4 of Ruscic, B., Active Thermochemical Tables: Water and Water Dimer. J. Phys. Chem. A 2013, 117 (46), 11940-11953. 
Table S15. The concentrations of water and water dimer at altitudes 1-17 km. (concentration unit: molecules $\left./ \mathrm{cm}^{3}\right)^{a}$

\begin{tabular}{|l|c|c|c|c|c|c|}
\hline $\begin{array}{c}\text { Altitude } \\
\mathrm{km}\end{array}$ & $\begin{array}{c}T^{b} \\
\mathrm{~K}\end{array}$ & $\begin{array}{c}P^{c} \\
\mathrm{mbar}\end{array}$ & {$\left[\mathrm{H}_{2} \mathrm{O}\right]^{c}$} & $K_{e q}{ }^{d}$ & {$\left[\left(\mathrm{H}_{2} \mathrm{O}\right)_{2}\right]$} & $\begin{array}{c}{\left[\left(\mathrm{H}_{2} \mathrm{O}\right)_{2}\right] /} \\
{\left[\mathrm{H}_{2} \mathrm{O}\right]}\end{array}$ \\
\hline 1 & 287.4 & 904 & $3.24 \mathrm{E}+17$ & $2.51 \mathrm{E}-21$ & $2.63 \mathrm{E}+14$ & $8.1 \mathrm{E}-04$ \\
\hline 3 & 283.6 & 713 & $1.42 \mathrm{E}+17$ & $2.71 \mathrm{E}-21$ & $5.47 \mathrm{E}+13$ & $3.8 \mathrm{E}-04$ \\
\hline 5 & 279.6 & 559 & $1.91 \mathrm{E}+16$ & $2.93 \mathrm{E}-21$ & $1.07 \mathrm{E}+12$ & $5.6 \mathrm{E}-05$ \\
\hline 7 & 255.5 & 432 & $3.71 \mathrm{E}+15$ & $5.08 \mathrm{E}-21$ & $7.00 \mathrm{E}+10$ & $1.9 \mathrm{E}-05$ \\
\hline 9 & 246.5 & 329 & $8.99 \mathrm{E}+14$ & $6.43 \mathrm{E}-21$ & $5.20 \mathrm{E}+09$ & $5.8 \mathrm{E}-06$ \\
\hline 11 & 228.3 & 247 & $7.37 \mathrm{E}+14$ & $1.10 \mathrm{E}-20$ & $5.99 \mathrm{E}+09$ & $8.1 \mathrm{E}-06$ \\
\hline 13 & 209.9 & 182 & $7.22 \mathrm{E}+13$ & $2.10 \mathrm{E}-20$ & $1.10 \mathrm{E}+08$ & $1.5 \mathrm{E}-06$ \\
\hline
\end{tabular}

${ }^{a}$ The main purpose of Table S15 is to indicate that although the temperatures, pressures, and water concentrations evaluated in various referecnes are not all the same, the differences do not change our conclusions.

${ }^{b}$ The data is from Palchetti, L.; Bianchini, G.; Carli, B.; Cortesi, U.; Del Bianco, S., Measurement of the water vapour vertical profile and of the Earth's outgoing far infrared flux. Atmos. Chem. Phys. 2008, 8, 2885-2894.) The temperature is from Table 2 of this reference; the water concentration is calculated from the mixing ratio in Table 3 of this reference and the ideal gas equation of state.

${ }^{c}$ McClatchey, R. A.; Fenn, R. W.; Selby, J. E. A.; Volz, F. E.; Garing, J. S. Optical Properties of the Atmosphere (Third Edition); Air Force Cambridge Research Laboratories Report AFCRL-72-0497 (Environmental Research Papers No. 411); Defense Technical Information Center: Bedford, MA, 1972.

${ }^{d} K_{e q}$ is the equilibrium constant (in $\mathrm{cm}^{3} /$ molecule) for the association of two water monomers to make a dimer; it is calculated by cubic spline data interpolation of Table S4 of Ruscic, B., Active Thermochemical Tables: Water and Water Dimer. J. Phys. Chem. A 2013, 117 (46), 11940-11953. 
Table S16. The rate constants at various temperatures and pressures

\begin{tabular}{lllcccccc}
\hline$H^{a}$ & $T^{a}$ & $P^{a}$ & $k_{\mathrm{B} 1 \mathrm{a}}$ & $k_{\mathrm{U} 1 \mathrm{c}}$ & $k_{\mathrm{B} 2}$ & $k_{\mathrm{U} 2 \mathrm{hs}}$ & $k_{\mathrm{B} 3 \mathrm{a}}$ & $k_{\mathrm{U} 3 \mathrm{c}}$ \\
$\mathrm{km}$ & $\mathrm{K}$ & mbar & & & & & & \\
\hline 0 & 288.8 & 1013 & $2.11 \mathrm{E}-16$ & $2.92 \mathrm{E}-2$ & $1.47 \mathrm{E}-19$ & $2.24 \mathrm{E}+2$ & $5.09 \mathrm{E}-15$ & $2.02 \mathrm{E}+1$ \\
5 & 259.3 & 542 & $1.30 \mathrm{E}-16$ & $6.85 \mathrm{E}-4$ & $6.42 \mathrm{E}-20$ & $6.45 \mathrm{E}+1$ & $4.75 \mathrm{E}-15$ & $2.92 \mathrm{E}-1$ \\
10 & 229.7 & 269 & $7.24 \mathrm{E}-17$ & $8.40 \mathrm{E}-6$ & $3.43 \mathrm{E}-20$ & $1.89 \mathrm{E}+1$ & $4.46 \mathrm{E}-15$ & $2.00 \mathrm{E}-2$ \\
15 & 212.6 & 122 & $4.87 \mathrm{E}-17$ & $3.75 \mathrm{E}-7$ & $2.92 \mathrm{E}-20$ & 9.35 & $4.32 \mathrm{E}-15$ & $1.41 \mathrm{E}-3$ \\
20 & 215.5 & 55 & $5.23 \mathrm{E}-17$ & $3.00 \mathrm{E}-7$ & $2.96 \mathrm{E}-20$ & $1.06 \mathrm{E}+1$ & $4.34 \mathrm{E}-15$ & $1.73 \mathrm{E}-3$ \\
25 & 218.6 & 25 & $5.63 \mathrm{E}-17$ & $2.25 \mathrm{E}-7$ & $3.02 \mathrm{E}-20$ & $1.20 \mathrm{E}+1$ & $4.36 \mathrm{E}-15$ & $1.81 \mathrm{E}-3$ \\
30 & 223.7 & 11.5 & $6.34 \mathrm{E}-17$ & $2.15 \mathrm{E}-7$ & $3.17 \mathrm{E}-20$ & $1.48 \mathrm{E}+1$ & $4.40 \mathrm{E}-15$ & $4.08 \mathrm{E}-3$ \\
35 & 235.1 & 5.4 & $8.13 \mathrm{E}-17$ & $4.67 \mathrm{E}-7$ & $3.74 \mathrm{E}-20$ & $2.36 \mathrm{E}+1$ & $4.50 \mathrm{E}-15$ & $3.86 \mathrm{E}-3$ \\
40 & 249.9 & 2.7 & $1.09 \mathrm{E}-16$ & $1.48 \mathrm{E}-6$ & $5.09 \mathrm{E}-20$ & $4.37 \mathrm{E}+1$ & $4.65 \mathrm{E}-15$ & $9.90 \mathrm{E}-3$ \\
45 & 266.1 & 1.4 & $1.46 \mathrm{E}-16$ & $4.98 \mathrm{E}-6$ & $7.70 \mathrm{E}-20$ & $8.47 \mathrm{E}+1$ & $4.83 \mathrm{E}-15$ & $2.59 \mathrm{E}-2$ \\
50 & 271.0 & 0.73 & $1.59 \mathrm{E}-16$ & $4.44 \mathrm{E}-6$ & $8.82 \mathrm{E}-20$ & $1.02 \mathrm{E}+2$ & $4.88 \mathrm{E}-15$ & $2.15 \mathrm{E}-2$ \\
\hline
\end{tabular}

${ }^{a}$ H, T, and $P$ from Brasseur, G.; Solomon, S., Aeronomy of the Middle Atmosphere: Chemistry and Physics of the Stratosphere and Mesosphere, Springer Netherlands: Dordrecht, 1986; pp. 441-442. This data is also used in Frost, G. J.; Vaida, V., Atmospheric implications of the photolysis of the ozone-water weakly bound complex. J.Geophys. Res., [Atmos.] 1995, 100 (D9), 18803-18809. 
Table S17. Cartesian coordinates $(\AA)$ of optimized structures

\begin{tabular}{|c|c|c|c|c|c|c|}
\hline Species & Methods & \multicolumn{5}{|c|}{ Cartesian coordinates } \\
\hline \multirow{42}{*}{$\mathrm{CH}_{2} \mathrm{OO}$} & \multirow{5}{*}{$\begin{array}{l}\text { CCSD(T)- } \\
\text { F12a } \\
/ \text { TZ-F12 }\end{array}$} & $\mathrm{C}$ & 1.0734406890 & -0.2039440088 & \multicolumn{2}{|c|}{0.0000000000} \\
\hline & & $\mathrm{O}$ & -0.0036139765 & 0.4692590146 & \multicolumn{2}{|c|}{0.0000000000} \\
\hline & & $\mathrm{O}$ & -1.1640249441 & -0.2043462143 & \multicolumn{2}{|c|}{0.0000000000} \\
\hline & & $\mathrm{H}$ & 1.0096510281 & -1.2842984575 & \multicolumn{2}{|c|}{0.0000000000} \\
\hline & & $\mathrm{H}$ & 1.9769172035 & 0.3865056659 & \multicolumn{2}{|c|}{0.0000000000} \\
\hline & $\operatorname{CCSD}(\mathrm{T})-$ & $\mathrm{C}$ & 1.0738898213 & -0.2040618152 & \multicolumn{2}{|c|}{0.0000000000} \\
\hline & $\mathrm{F} 12 \mathrm{a}$ & $\mathrm{O}$ & -0.0046664679 & 0.4706154646 & \multicolumn{2}{|c|}{0.0000000000} \\
\hline & /DZ-F12 & $\mathrm{O}$ & -1.1647544521 & -0.2048945578 & \multicolumn{2}{|c|}{0.0000000000} \\
\hline & & $\mathrm{H}$ & 1.0101594984 & -1.2843811341 & \multicolumn{2}{|c|}{0.0000000000} \\
\hline & & $\mathrm{H}$ & 1.9777416003 & 0.3858980425 & \multicolumn{2}{|c|}{0.0000000000} \\
\hline & QCISD & $\mathrm{C}$ & 1.064286 & -0.208194 & \multicolumn{2}{|c|}{0.000000} \\
\hline & $/ \mathrm{TZ}$ & $\mathrm{O}$ & 0.000000 & 0.477756 & \multicolumn{2}{|c|}{0.000000} \\
\hline & & $\mathrm{O}$ & -1.169255 & -0.205758 & \multicolumn{2}{|c|}{0.000000} \\
\hline & & $\mathrm{H}$ & 0.986359 & -1.286839 & \multicolumn{2}{|l|}{0.000000} \\
\hline & & $\mathrm{H}$ & 1.981965 & 0.360014 & \multicolumn{2}{|l|}{0.000000} \\
\hline & MN15-L & $\mathrm{C}$ & 1.068365 & -0.208923 & \multicolumn{2}{|c|}{0.000000} \\
\hline & /MG3S & $\mathrm{O}$ & 0.000000 & 0.458735 & \multicolumn{2}{|c|}{0.000000} \\
\hline & & $\mathrm{O}$ & -1.176397 & -0.187276 & \multicolumn{2}{|c|}{0.000000} \\
\hline & & $\mathrm{H}$ & 1.015852 & -1.303984 & \multicolumn{2}{|c|}{0.000000} \\
\hline & & $\mathrm{H}$ & 1.985134 & 0.385850 & \multicolumn{2}{|c|}{0.000000} \\
\hline & MN15-L & $\mathrm{C}$ & 1.068968 & -0.206388 & \multicolumn{2}{|c|}{0.000000} \\
\hline & /maug-TZ & $\mathrm{O}$ & 0.000000 & 0.460328 & 0.00000 & \\
\hline & & $\mathrm{O}$ & -1.176930 & -0.191553 & 0.00000 & \\
\hline & & $\mathrm{H}$ & 1.017004 & -1.300634 & 0.0000 & \\
\hline & & $\mathrm{H}$ & 1.984628 & 0.388762 & 0.0000 & \\
\hline & M11-L & $\mathrm{C}$ & & 1.039537 & -0.218128 & 0.000000 \\
\hline & /MG3S & $\mathrm{O}$ & & 0.000000 & 0.450586 & 0.000000 \\
\hline & & $\mathrm{O}$ & & -1.146903 & -0.168543 & 0.000000 \\
\hline & & $\mathrm{H}$ & & 0.976111 & -1.310702 & 0.000000 \\
\hline & & $\mathrm{H}$ & & 1.961895 & 0.363130 & 0.000000 \\
\hline & M11-L & $\mathrm{C}$ & & 1.041583 & -0.216075 & 0.000000 \\
\hline & /maug-TZ & $\mathrm{O}$ & & 0.000000 & 0.452584 & 0.000000 \\
\hline & & $\mathrm{O}$ & & -1.148829 & -0.172805 & 0.000000 \\
\hline & & $\mathrm{H}$ & & 0.979110 & -1.307040 & 0.000000 \\
\hline & & $\mathrm{H}$ & & 1.962026 & 0.365258 & 0.000000 \\
\hline & M06-L & $\mathrm{C}$ & & 1.067302 & -0.213740 & 0.000000 \\
\hline & /MG3S & ( & & 0.000000 & 0.455009 & 0.000000 \\
\hline & & ( & & -1.175011 & -0.179397 & 0.000000 \\
\hline & & $\mathrm{I}$ & & 1.022422 & -1.297210 & 0.000000 \\
\hline & & $\underline{\mathrm{H}}$ & & 1.973859 & 0.374756 & 0.000000 \\
\hline & M06-L & $\mathrm{C}$ & & 1.067541 & -0.211676 & 0.000000 \\
\hline & /maug-TZ & ( & & 0.000000 & 0.456936 & 0.000000 \\
\hline
\end{tabular}




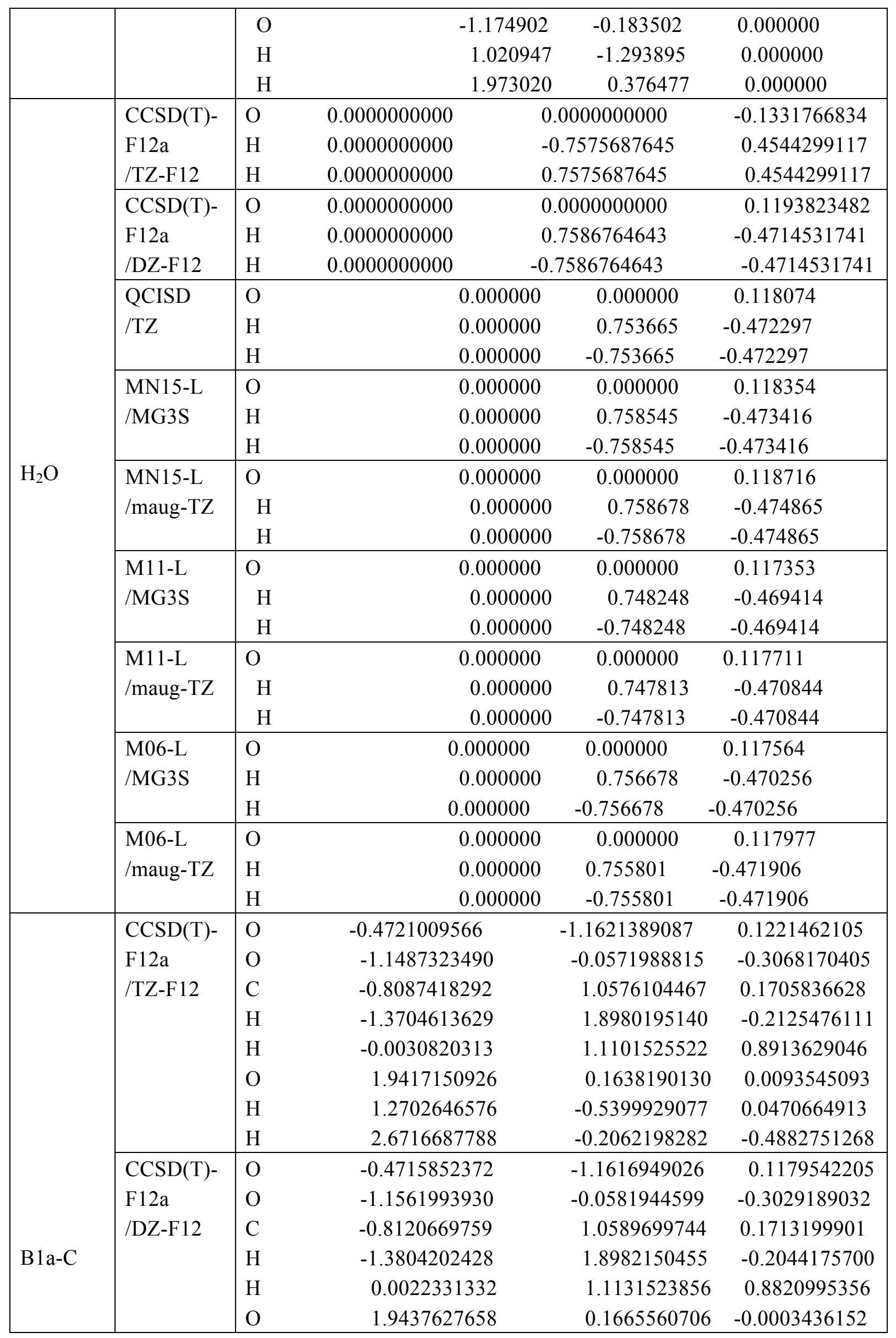




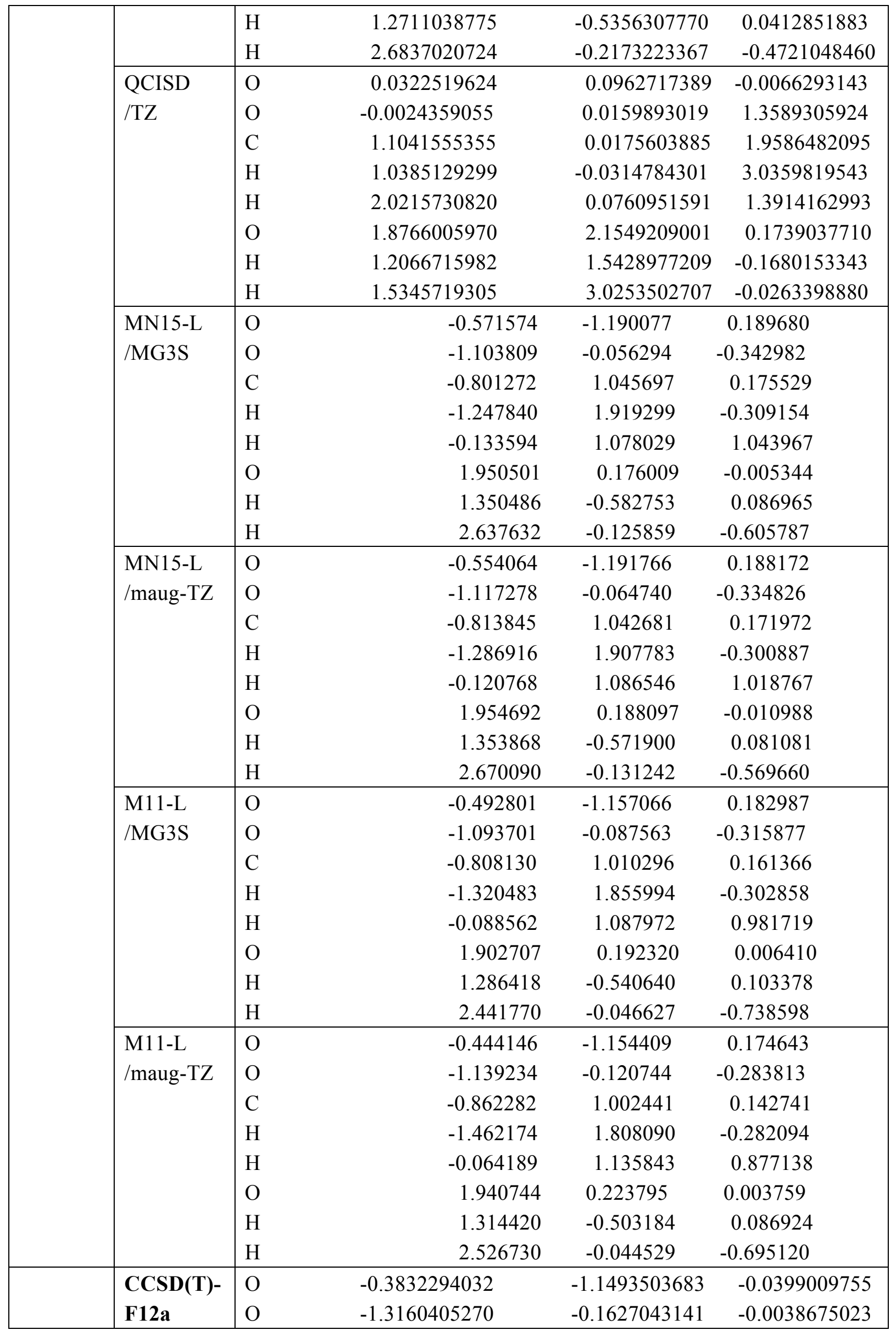




\begin{tabular}{|c|c|c|c|c|c|}
\hline \multirow{22}{*}{ B1dh-C } & \multirow[t]{6}{*}{ /TZ-F12 } & $\mathrm{C}$ & -0.9307888810 & 1.0405528736 & -0.0325474578 \\
\hline & & $\mathrm{H}$ & -1.7391357295 & 1.7573740028 & -0.0009418307 \\
\hline & & $\mathrm{H}$ & 0.1279487756 & 1.2728070200 & -0.0754039603 \\
\hline & & $\mathrm{O}$ & 1.9880741470 & 0.2955116745 & 0.1551621375 \\
\hline & & $\mathrm{H}$ & 1.3389476292 & -0.4271871493 & 0.1050457638 \\
\hline & & $\mathrm{H}$ & 2.8265209891 & -0.1101217393 & 0.3775808248 \\
\hline & \multirow{8}{*}{$\begin{array}{l}\text { CCSD(T)- } \\
\text { F12a } \\
\text { /DZ-F12 }\end{array}$} & $\mathrm{O}$ & -0.3822517339 & -1.1499196825 & -0.0398883482 \\
\hline & & $\mathrm{O}$ & -1.3162130140 & -0.1638990472 & 0.0056936929 \\
\hline & & $\mathrm{C}$ & -0.9304214925 & 1.0410735860 & -0.0297810633 \\
\hline & & $\mathrm{H}$ & -1.7381791142 & 1.7582926148 & 0.0071992263 \\
\hline & & $\mathrm{H}$ & 0.1279578116 & 1.2726925189 & -0.0808264867 \\
\hline & & $\mathrm{O}$ & 1.9978237529 & 0.2905226920 & 0.1141263214 \\
\hline & & $\mathrm{H}$ & 1.3415743887 & -0.4266347686 & 0.0820013706 \\
\hline & & $\mathrm{H}$ & 2.8120064015 & -0.1052459134 & 0.4266022867 \\
\hline & \multirow{8}{*}{$\begin{array}{l}\text { QCISD } \\
/ \mathrm{TZ}\end{array}$} & $\mathrm{O}$ & -0.393480 & -1.162165 & -0.001618 \\
\hline & & $\mathrm{O}$ & -1.314579 & -0.157267 & 0.003663 \\
\hline & & $\mathrm{C}$ & -0.896208 & 1.034863 & 0.013592 \\
\hline & & $\mathrm{H}$ & -1.679638 & 1.778801 & 0.013460 \\
\hline & & $\mathrm{H}$ & 0.169784 & 1.232708 & 0.024853 \\
\hline & & $\mathrm{O}$ & 2.059135 & 0.229265 & -0.081036 \\
\hline & & $\mathrm{H}$ & 1.371671 & -0.453788 & -0.055237 \\
\hline & & $\mathrm{H}$ & 2.706826 & -0.045558 & 0.567303 \\
\hline & $\operatorname{CCSD}(\mathrm{T})-$ & $\mathrm{C}$ & 0.0352086055 & 979366476 & 0.2461706019 \\
\hline & $\mathrm{F} 12 \mathrm{a} / \mathrm{TZ}-\mathrm{F}$ & $\mathrm{O}$ & -0.8863650562 & 943912770 & -0.3933287530 \\
\hline & 12 & $\mathrm{O}$ & -0.9520015998 & 478974338 & 0.1862672532 \\
\hline & & $\mathrm{H}$ & 0.1648656251 & 02894026 & 1.3035001914 \\
\hline & & $\mathrm{H}$ & 0.3899524064 & 13070745 & -0.2038568970 \\
\hline & & $\mathrm{O}$ & 1.4508931019 & 39359120 & -0.0070205673 \\
\hline & & $\mathrm{H}$ & 0.5815378175 & 11347402 & 0.0827664704 \\
\hline & & $\mathrm{H}$ & 1.6840480996 & 9723158 & -0.9436422996 \\
\hline & $\operatorname{CCSD}(\mathrm{T})-$ & $\mathrm{C}$ & 0.0381936679 & 932001077 & 0.2449940302 \\
\hline & F12a/DZ-F & $\mathrm{O}$ & -0.8846256826 & 924794607 & -0.3899869679 \\
\hline & 12 & $\mathrm{O}$ & -0.9507938116 & 430865160 & 0.1841981698 \\
\hline & & $\mathrm{H}$ & 0.1659755276 & 992178150 & 1.3018890528 \\
\hline & & $\mathrm{H}$ & 0.3903728820 & 156745649 & -0.2053050316 \\
\hline & & $\mathrm{O}$ & 1.4457909897 & 496523617 & -0.0077770581 \\
\hline & & $\mathrm{H}$ & 0.5802801147 & 308305950 & 0.0833958098 \\
\hline & & $\mathrm{H}$ & 1.6829453123 & 910184757 & -0.9405520052 \\
\hline & QCISD/TZ & $\mathrm{C}$ & 0.076700 & 0.979004 & 0.254816 \\
\hline B1a-TS1 & & $\mathrm{O}$ & -0.867842 & 0.414787 & -0.385890 \\
\hline & & $\mathrm{O}$ & -0.970292 & -0.916814 & 0.171665 \\
\hline & & $\mathrm{H}$ & 0.187446 & 0.783578 & 1.311795 \\
\hline & & $\mathrm{H}$ & 0.467535 & 1.889015 & -0.185864 \\
\hline & & $\mathrm{O}$ & 1.424658 & -0.393883 & -0.009380 \\
\hline
\end{tabular}




\begin{tabular}{|c|c|c|c|c|}
\hline & $\begin{array}{l}\mathrm{H} \\
\mathrm{H} \\
\end{array}$ & $\begin{array}{l}0.541005 \\
1.651619 \\
\end{array}$ & $\begin{array}{l}-0.945290 \\
-0.434038 \\
\end{array}$ & $\begin{array}{r}0.077144 \\
-0.943129 \\
\end{array}$ \\
\hline \multirow{8}{*}{$\begin{array}{l}\text { MN15-L/ } \\
\text { MG3S }\end{array}$} & $\mathrm{C}$ & 0.127873 & 0.958129 & 0.261145 \\
\hline & $\mathrm{O}$ & -0.847131 & 0.445743 & -0.389168 \\
\hline & $\mathrm{O}$ & -1.025350 & -0.879423 & 0.169356 \\
\hline & $\mathrm{H}$ & 0.208505 & 0.768263 & 1.338753 \\
\hline & $\mathrm{H}$ & 0.570946 & 1.862870 & -0.180552 \\
\hline & $\mathrm{O}$ & 1.412609 & -0.435637 & -0.011116 \\
\hline & $\mathrm{H}$ & 0.523647 & -0.980828 & 0.080355 \\
\hline & $\mathrm{H}$ & 1.608638 & -0.444545 & -0.958005 \\
\hline \multirow{8}{*}{$\begin{array}{l}\text { MN15-L } \\
/ \text { maug-TZ }\end{array}$} & $\mathrm{C}$ & 0.101573 & 0.968733 & 0.260667 \\
\hline & $\mathrm{O}$ & -0.855337 & 0.431301 & -0.391452 \\
\hline & $\mathrm{O}$ & -1.014811 & -0.894575 & 0.170164 \\
\hline & $\mathrm{H}$ & 0.193806 & 0.771572 & 1.334956 \\
\hline & $\mathrm{H}$ & 0.526495 & 1.880553 & -0.182173 \\
\hline & $\mathrm{O}$ & 1.430626 & -0.419145 & -0.008935 \\
\hline & $\mathrm{H}$ & 0.551766 & -0.972151 & 0.079592 \\
\hline & $\mathrm{H}$ & 1.634671 & -0.433011 & -0.954587 \\
\hline \multirow{8}{*}{$\begin{array}{l}\text { M11-L } \\
\text { /MG3S }\end{array}$} & $\mathrm{C}$ & 0.073141 & 0.955824 & 0.251228 \\
\hline & $\mathrm{O}$ & -0.847916 & 0.403582 & -0.383145 \\
\hline & $\mathrm{O}$ & -0.969101 & -0.889777 & 0.167847 \\
\hline & $\mathrm{H}$ & 0.184287 & 0.759300 & 1.323721 \\
\hline & $\mathrm{H}$ & 0.466133 & 1.884581 & -0.185596 \\
\hline & $\mathrm{O}$ & 1.409987 & -0.391499 & -0.007713 \\
\hline & $\mathrm{H}$ & 0.547169 & -0.947292 & 0.077454 \\
\hline & $\mathrm{H}$ & 1.619799 & -0.409982 & -0.938867 \\
\hline \multirow{8}{*}{$\begin{array}{l}\text { M11-L } \\
\text { /maug-TZ }\end{array}$} & $\mathrm{C}$ & 0.053670 & 0.962663 & 0.252359 \\
\hline & $\mathrm{O}$ & -0.855076 & 0.395747 & -0.385634 \\
\hline & $\mathrm{O}$ & -0.960830 & -0.903081 & 0.168672 \\
\hline & $\mathrm{H}$ & 0.172061 & 0.760546 & 1.321172 \\
\hline & $\mathrm{H}$ & 0.438071 & 1.893870 & -0.183187 \\
\hline & $\mathrm{O}$ & 1.423417 & -0.379032 & -0.006762 \\
\hline & $\mathrm{H}$ & 0.569138 & -0.940676 & 0.074561 \\
\hline & $\mathrm{H}$ & 1.638617 & -0.398796 & -0.936902 \\
\hline \multirow{8}{*}{$\begin{array}{l}\text { M06-L } \\
\text { /MG3S }\end{array}$} & $\mathrm{C}$ & 0.126074 & 0.966612 & 0.250328 \\
\hline & $\mathrm{O}$ & -0.859672 & 0.444593 & -0.373532 \\
\hline & $\mathrm{O}$ & -0.989985 & -0.898361 & 0.167012 \\
\hline & $\mathrm{H}$ & 0.231080 & 0.802284 & 1.317655 \\
\hline & $\mathrm{H}$ & 0.531324 & 1.871647 & -0.196389 \\
\hline & $\mathrm{O}$ & 1.399384 & -0.428433 & -0.012276 \\
\hline & $\mathrm{H}$ & 0.469487 & -0.972786 & 0.078287 \\
\hline & $\mathrm{H}$ & 1.613848 & -0.443201 & -0.951161 \\
\hline M06-L & $\mathrm{C}$ & 0.116154 & 0.969613 & 0.251928 \\
\hline /maug-TZ & $\mathrm{O}$ & -0.862305 & 0.440687 & -0.375829 \\
\hline
\end{tabular}




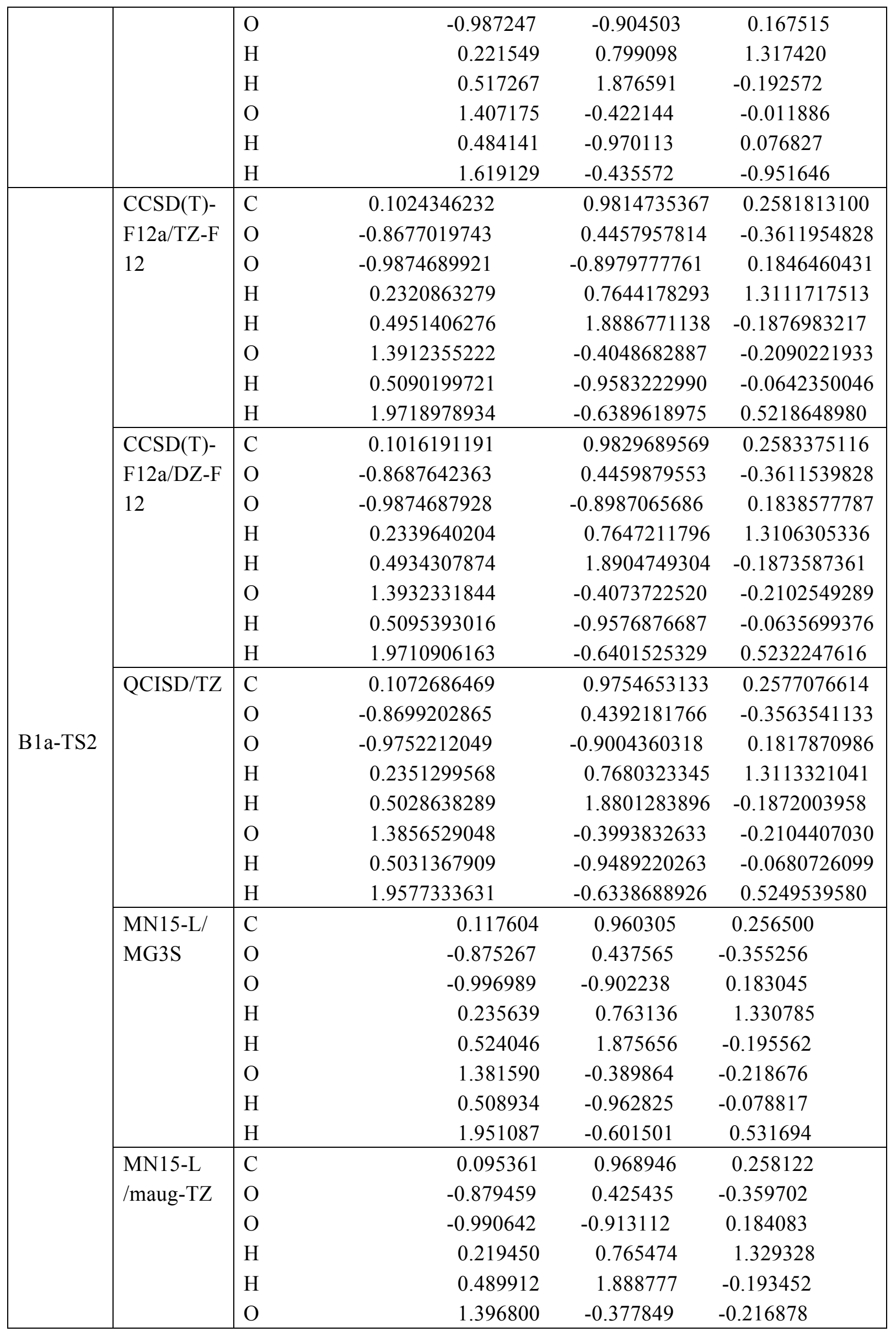




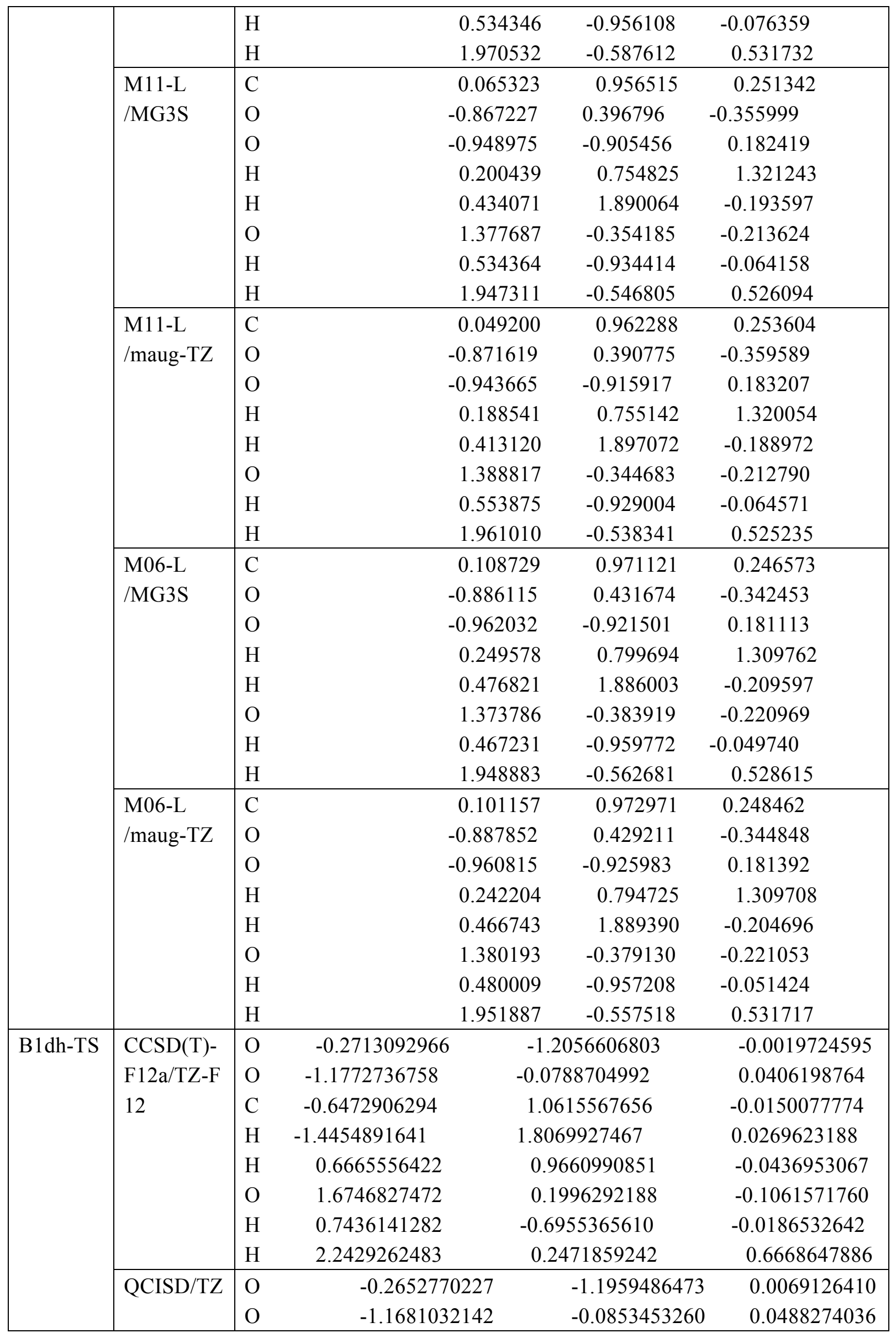




\begin{tabular}{|c|c|c|c|c|c|}
\hline & & $\mathrm{C}$ & -0.6428945535 & 1.0616698180 & -0.0103484928 \\
\hline & & $\mathrm{H}$ & -1.4414966898 & 1.8046036481 & 0.0283055813 \\
\hline & & $\mathrm{H}$ & 0.6715933564 & 0.9594646424 & -0.0553141211 \\
\hline & & $\mathrm{O}$ & 1.6729178070 & 0.1959972714 & -0.1189588339 \\
\hline & & $\mathrm{H}$ & 0.7564233614 & -0.6761125021 & -0.0298555573 \\
\hline & & $\mathrm{H}$ & 2.2032529555 & 0.2370670954 & 0.6793923792 \\
\hline \multirow[t]{16}{*}{ U1c-TS } & $\operatorname{CCSD}(\mathrm{T})-$ & $\mathrm{O}$ & -0.8831764983 & -0.5875817698 & 0.2793545435 \\
\hline & F12a/TZ-F & $\mathrm{C}$ & 0.2936286156 & -0.6575748558 & -0.2322999360 \\
\hline & 12 & $\mathrm{O}$ & -1.0313188253 & 0.8049828926 & -0.1328474479 \\
\hline & & $\mathrm{H}$ & 0.5357346789 & -1.5363245842 & -0.8262938067 \\
\hline & & $\mathrm{H}$ & 1.0128370390 & 0.1192829974 & -0.0112529428 \\
\hline & $\operatorname{CCSD}(\mathrm{T})-$ & $\mathrm{O}$ & -0.8840791913 & -0.5858419710 & 0.2826513088 \\
\hline & F12a/DZ-F & $\mathrm{C}$ & 0.2951548321 & -0.6576067532 & -0.2319604517 \\
\hline & 12 & $\mathrm{O}$ & -1.0336621625 & 0.8034142990 & -0.1354043068 \\
\hline & & $\mathrm{H}$ & 0.5348104059 & \multirow{2}{*}{$\begin{array}{r}-1.5349450161 \\
0.1177641214\end{array}$} & -0.8286060244 \\
\hline & & $\mathrm{H}$ & 1.0154811259 & & \\
\hline & & \multicolumn{2}{|c|}{-0.0100201159} & & \\
\hline & MN15-L & $\mathrm{O}$ & 0.058872 & \multirow{5}{*}{$\begin{array}{r}0.655663 \\
-0.282357 \\
-0.278779 \\
-0.116353 \\
-1.204577\end{array}$} & 0.084869 \\
\hline & /maug-TZ & $\mathrm{C}$ & 0.910980 & & 0.001280 \\
\hline & & $\mathrm{O}$ & -1.064318 & & 0.077291 \\
\hline & & $\mathrm{H}$ & 1.806502 & & 628069 \\
\hline & & $\mathrm{H}$ & 0.771189 & & -0.575129 \\
\hline \multirow[t]{22}{*}{ U1d-TS } & $\operatorname{CCSD}(\mathrm{T})-$ & $\mathrm{C}$ & 1.0320345766 & -0.1134844000 & \\
\hline & F12a/TZ-F & & & & \\
\hline & 12 & $\mathrm{O}$ & -0.0018792133 & 0.6016423369 & 0.0000000000 \\
\hline & & $\mathrm{O}$ & -1.0344265131 & -0.4393430123 & 0.0000000000 \\
\hline & & $\mathrm{H}$ & 0.1263757293 & -1.1072994717 & 0.0000000000 \\
\hline & & $\mathrm{H}$ & 1.9585704204 & 0.4728045473 & 0.0000000000 \\
\hline & $\operatorname{CCSD}(\mathrm{T})-$ & $\mathrm{C}$ & 1.0324901920 & -0.1137922500 & \\
\hline & F12a/DZ-F & & & & \\
\hline & 12 & $\mathrm{O}$ & -0.0025641334 & 0.6020160043 & 0.0000000000 \\
\hline & & $\mathrm{O}$ & -1.0352094827 & -0.4390130695 & 0.0000000000 \\
\hline & & $\mathrm{H}$ & 0.1270747626 & -1.1076755848 & 0.0000000000 \\
\hline & & $\mathrm{H}$ & 1.9588836616 & 0.4727849000 & 0.0000000000 \\
\hline & MN15-L & $\mathrm{C}$ & 1.032560 & -0.128726 & 000000 \\
\hline & /maug-TZ & $\mathrm{O}$ & 0.000000 & 0.593640 & 00000 \\
\hline & & $\mathrm{O}$ & -1.035125 & -0.416464 & 0000 \\
\hline & & $\mathrm{H}$ & 0.113091 & -1.115504 & 000000 \\
\hline & & $\mathrm{H}$ & 1.972548 & 0.470454 & 000000 \\
\hline & $\operatorname{CCSD}(\mathrm{T})-$ & $\mathrm{O}$ & -1.4574646887 & -0.4312859597 & 0.1694601947 \\
\hline & $\mathrm{F} 12 \mathrm{a} / \mathrm{TZ}-\mathrm{F}$ & $\mathrm{O}$ & -0.6200708980 & 0.6021876610 & -0.3977301721 \\
\hline & 12 & $\mathrm{C}$ & 0.6028226200 & 0.5471878330 & 0.2868236505 \\
\hline & & $\mathrm{H}$ & 1.1138319681 & 1.4605191558 & -0.0266244786 \\
\hline & & $\mathrm{H}$ & 0.4319933819 & 0.5282570766 & 1.3611436336 \\
\hline
\end{tabular}




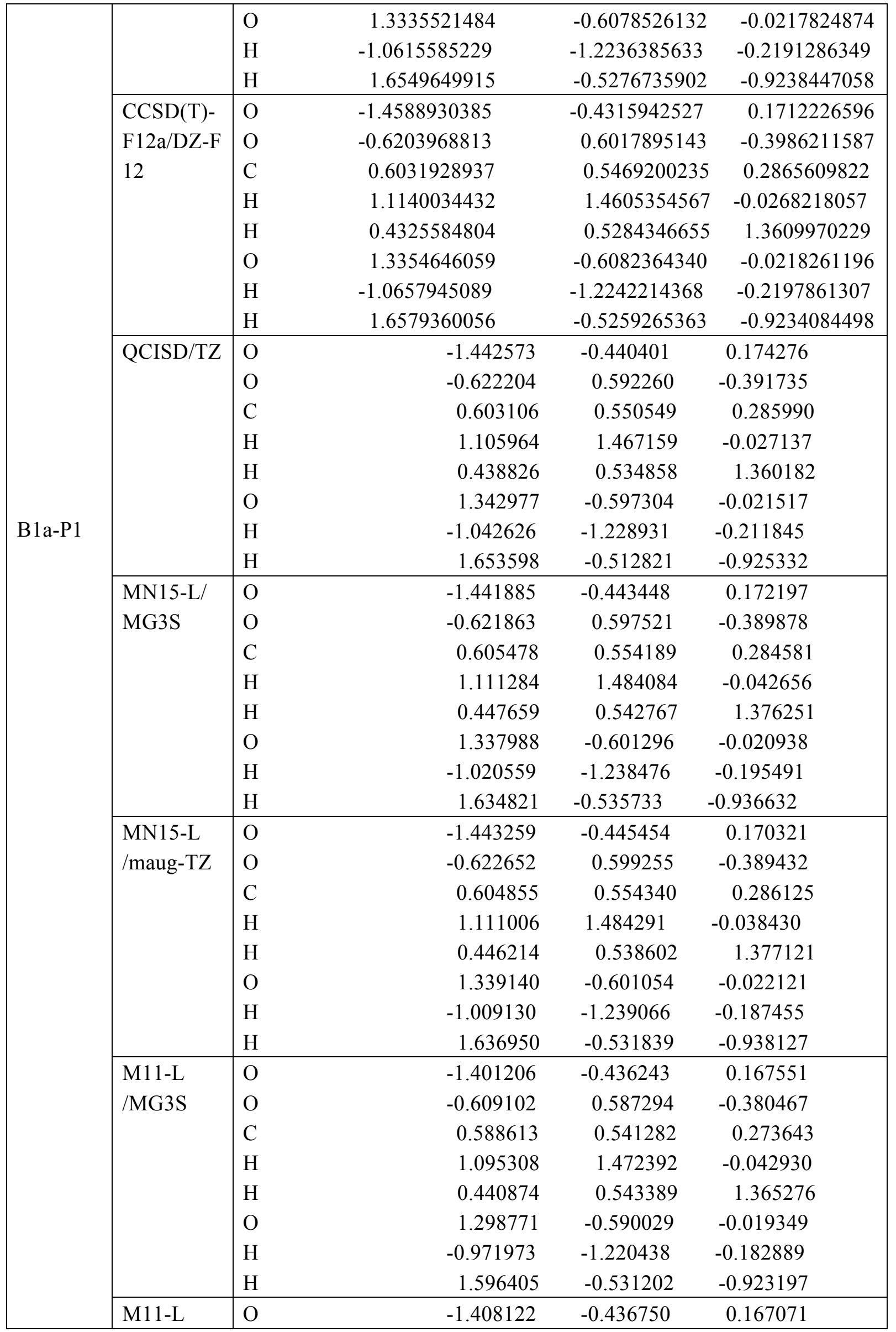




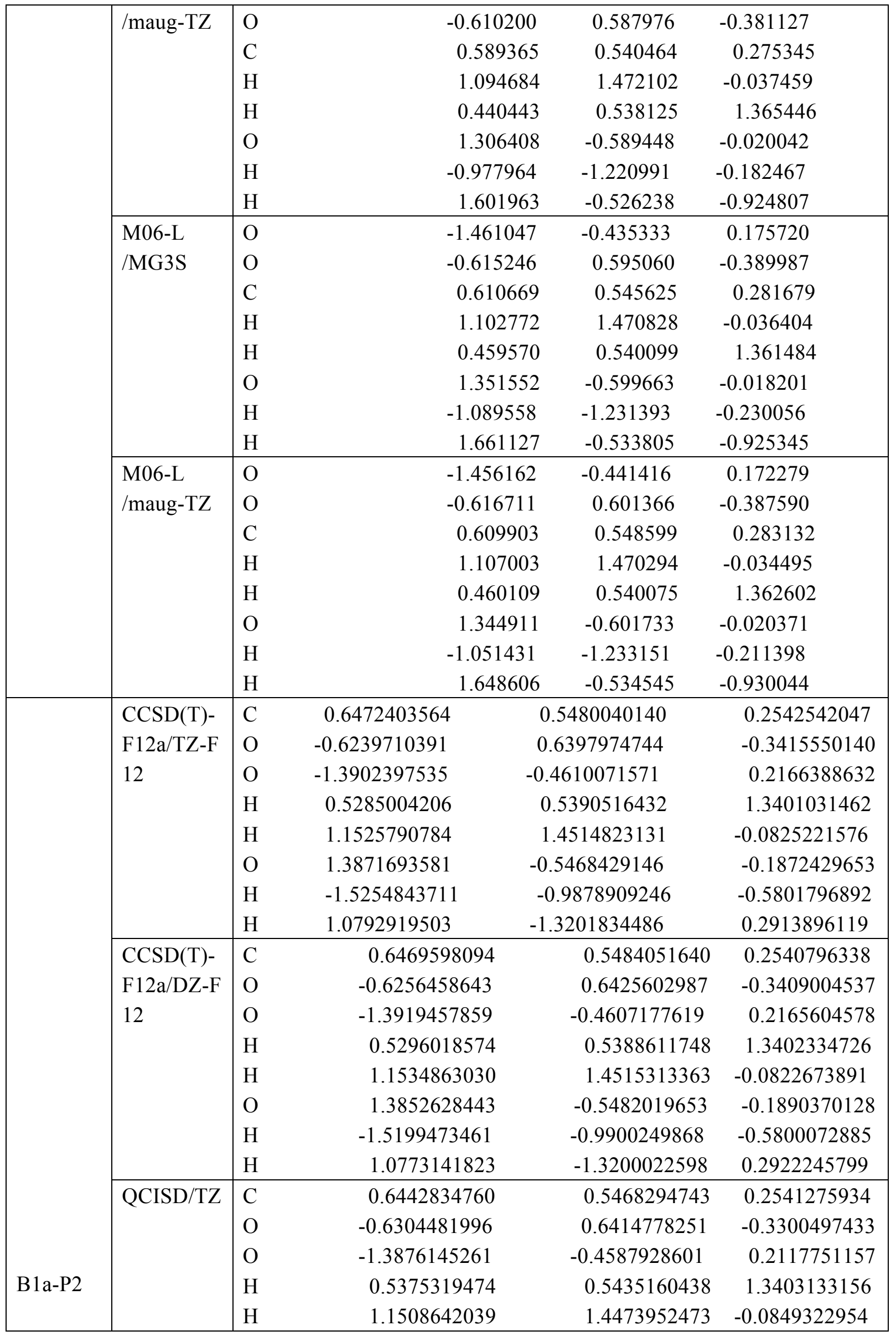




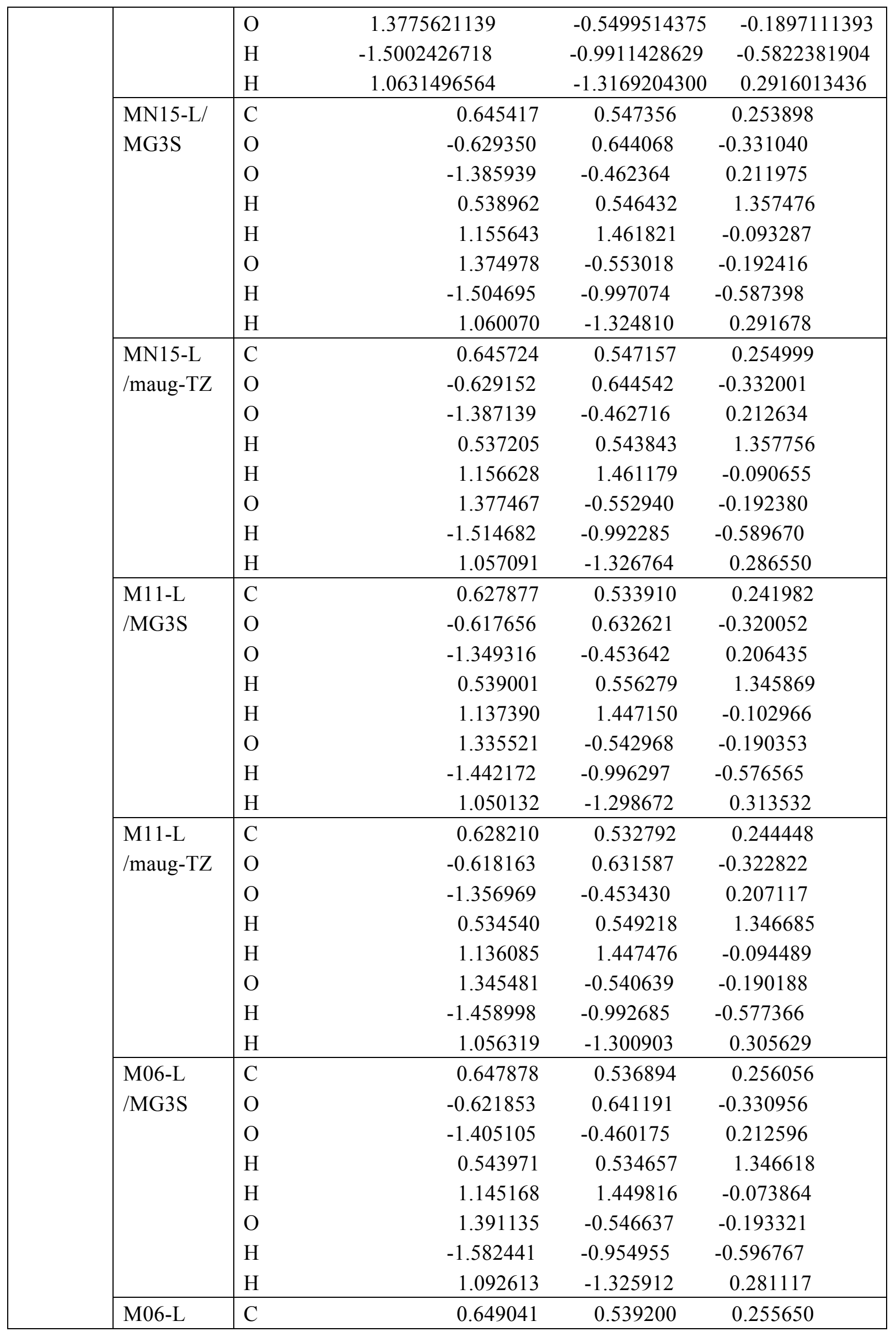




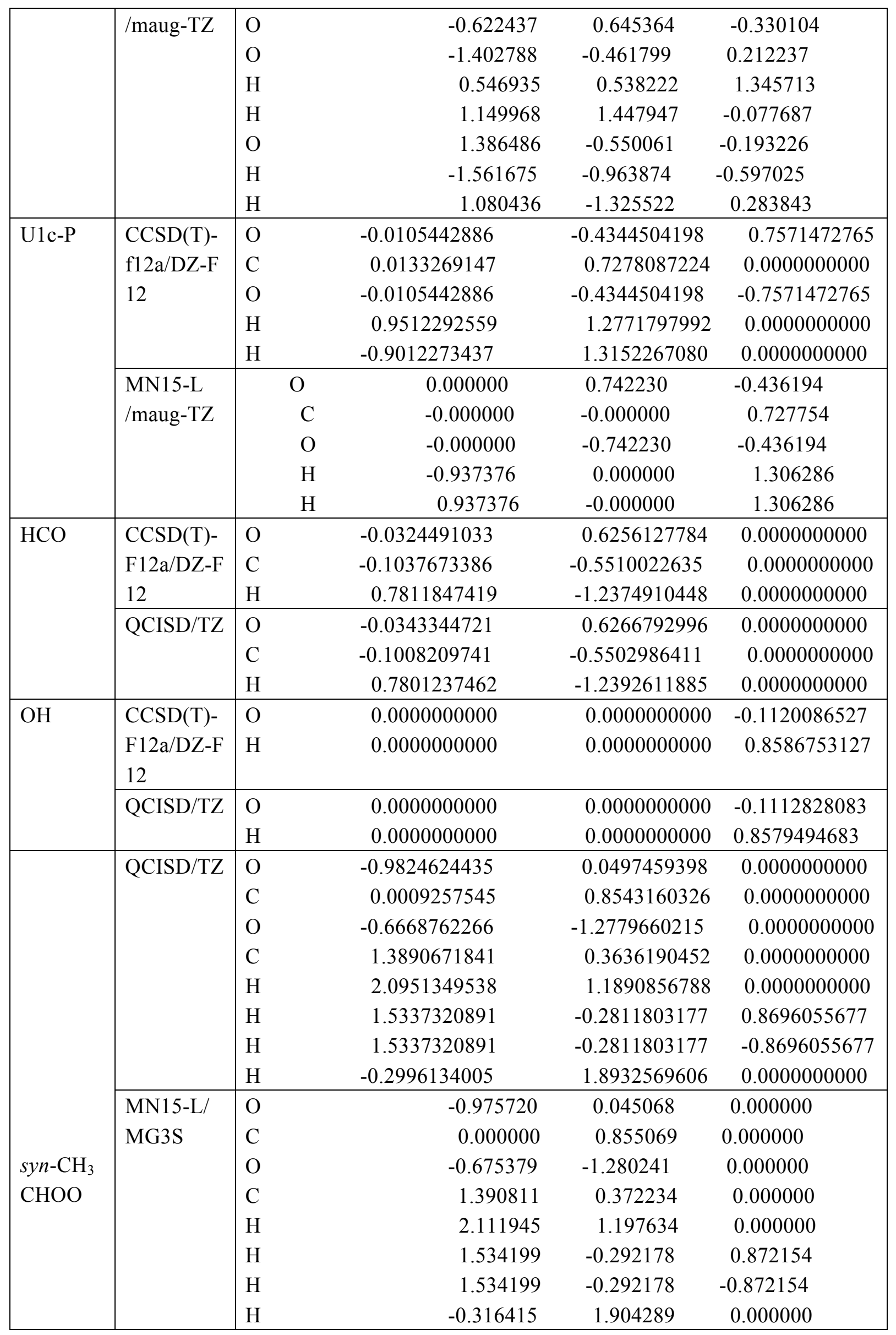




\begin{tabular}{|c|c|c|c|c|c|}
\hline & $\begin{array}{l}\text { MN15-L } \\
\text { /maug-TZ }\end{array}$ & $\begin{array}{l}\mathrm{O} \\
\mathrm{C} \\
\mathrm{O} \\
\mathrm{C} \\
\mathrm{H} \\
\mathrm{H} \\
\mathrm{H} \\
\mathrm{H} \\
\end{array}$ & $\begin{array}{r}-0.976918 \\
0.000000 \\
-0.673445 \\
1.390325 \\
2.111524 \\
1.531796 \\
1.531796 \\
-0.314163 \\
\end{array}$ & $\begin{array}{c}0.046754 \\
0.855460 \\
-1.281303 \\
0.371476 \\
1.196630 \\
-0.293099 \\
-0.293099 \\
1.904347 \\
\end{array}$ & $\begin{array}{c}0.000000 \\
0.000000 \\
0.000000 \\
0.000000 \\
0.000000 \\
0.872172 \\
-0.872172 \\
0.000000 \\
\end{array}$ \\
\hline & $\begin{array}{l}\text { M11-L/M } \\
\text { G3S }\end{array}$ & $\begin{array}{l}\mathrm{O} \\
\mathrm{C} \\
\mathrm{O} \\
\mathrm{C} \\
\mathrm{H} \\
\mathrm{H} \\
\mathrm{H} \\
\mathrm{H}\end{array}$ & $\begin{array}{r}-0.962573 \\
0.000000 \\
-0.653466 \\
1.358303 \\
2.090093 \\
1.496888 \\
1.496888 \\
-0.305380\end{array}$ & $\begin{array}{c}0.048495 \\
0.836027 \\
-1.237025 \\
0.348071 \\
1.156712 \\
-0.319518 \\
-0.319518 \\
1.885974\end{array}$ & $\begin{array}{c}0.000000 \\
0.000000 \\
0.000000 \\
0.000000 \\
0.000000 \\
0.863907 \\
-0.863907 \\
0.000000\end{array}$ \\
\hline & $\begin{array}{l}\text { M11-L } \\
\text { /maug-TZ }\end{array}$ & $\begin{array}{l}\mathrm{O} \\
\mathrm{C} \\
\mathrm{O} \\
\mathrm{C} \\
\mathrm{H} \\
\mathrm{H} \\
\mathrm{H} \\
\mathrm{H}\end{array}$ & $\begin{array}{r}-0.964672 \\
0.000000 \\
-0.652037 \\
1.359359 \\
2.091235 \\
1.494613 \\
1.494613 \\
-0.302941\end{array}$ & $\begin{array}{c}0.050485 \\
0.837490 \\
-1.239752 \\
0.347975 \\
1.154832 \\
-0.319933 \\
-0.319933 \\
1.886383\end{array}$ & $\begin{array}{c}0.000000 \\
0.000000 \\
0.000000 \\
0.000000 \\
0.000000 \\
0.862766 \\
-0.862766 \\
0.000000\end{array}$ \\
\hline & QCISD/TZ & $\begin{array}{l}\mathrm{O} \\
\mathrm{C} \\
\mathrm{O} \\
\mathrm{C} \\
\mathrm{H} \\
\mathrm{H} \\
\mathrm{H} \\
\mathrm{H} \\
\mathrm{O} \\
\mathrm{H} \\
\mathrm{H}\end{array}$ & $\begin{array}{r}-0.631683 \\
-1.109902 \\
0.105872 \\
-0.941780 \\
-1.604771 \\
-1.125614 \\
0.099803 \\
-1.658398 \\
2.048310 \\
1.573075 \\
2.846002\end{array}$ & $\begin{array}{c}-0.980890 \\
0.180320 \\
-1.207914 \\
1.241071 \\
2.074999 \\
0.816206 \\
1.570388 \\
0.294751 \\
0.535146 \\
-0.195781 \\
0.140352 \\
\end{array}$ & $\begin{array}{c}-0.432756 \\
-0.568840 \\
0.710951 \\
0.437125 \\
0.224401 \\
1.424513 \\
0.421256 \\
-1.495536 \\
-0.208674 \\
0.217834 \\
-0.558353\end{array}$ \\
\hline B2-C & $\begin{array}{l}\text { MN15-L/ } \\
\text { MG3S }\end{array}$ & $\begin{array}{l}\mathrm{O} \\
\mathrm{C} \\
\mathrm{O} \\
\mathrm{C} \\
\mathrm{H} \\
\mathrm{H} \\
\mathrm{H} \\
\mathrm{H} \\
\mathrm{O}\end{array}$ & $\begin{array}{c}-0.548661 \\
-1.016370 \\
-0.004295 \\
-1.031878 \\
-1.581511 \\
-1.461206 \\
0.010420 \\
-1.413301 \\
2.066586\end{array}$ & $\begin{array}{c}-0.944756 \\
0.222521 \\
-1.258090 \\
1.198170 \\
2.105530 \\
0.708949 \\
1.443262 \\
0.401922 \\
0.548654\end{array}$ & $\begin{array}{c}-0.504102 \\
-0.635905 \\
0.718620 \\
0.465886 \\
0.190016 \\
1.358231 \\
0.739838 \\
-1.642964 \\
-0.155188\end{array}$ \\
\hline
\end{tabular}




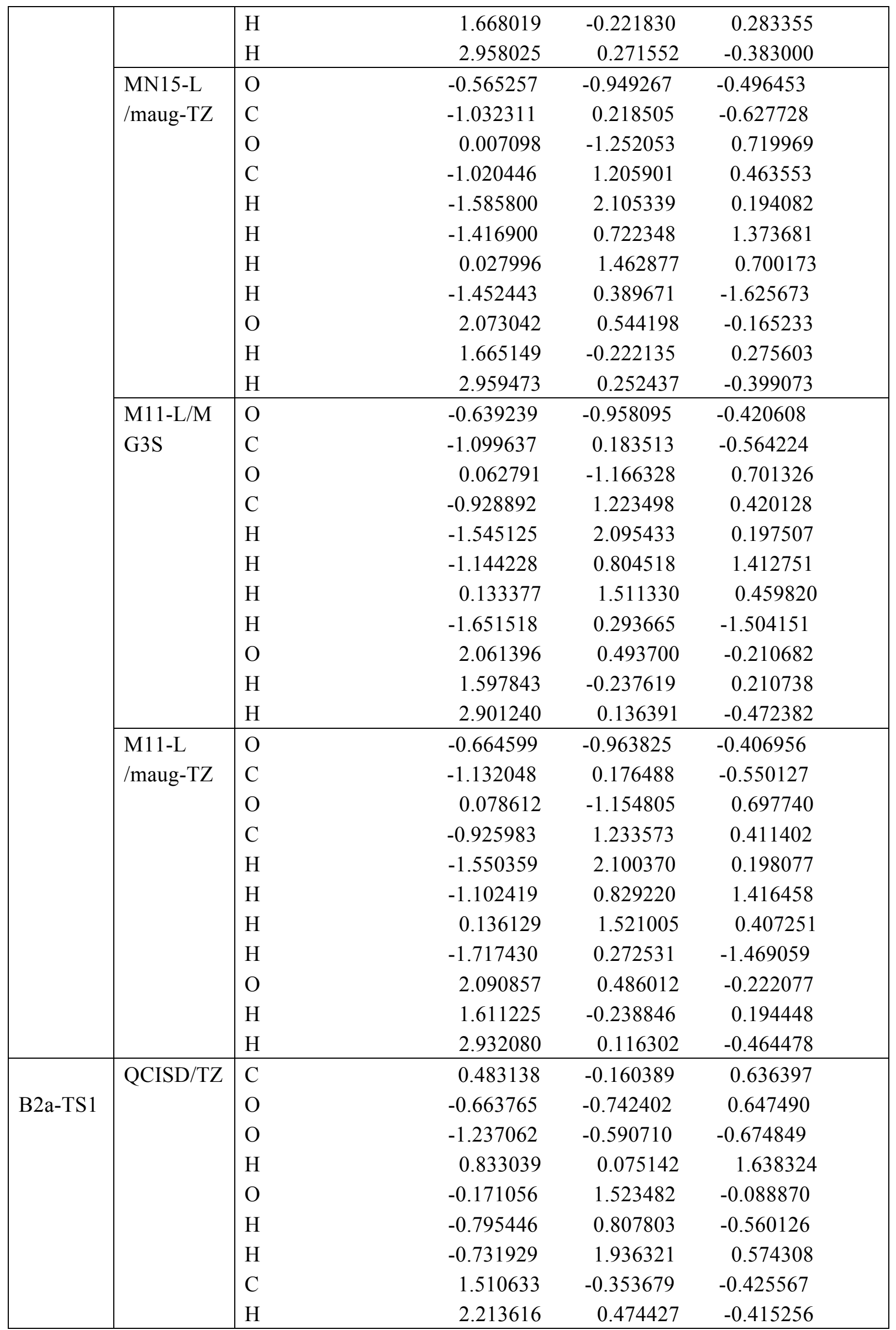




\begin{tabular}{|c|c|c|c|c|}
\hline & $\begin{array}{l}\mathrm{H} \\
\mathrm{H} \\
\end{array}$ & $\begin{array}{l}2.045983 \\
1.047173 \\
\end{array}$ & $\begin{array}{l}-1.273320 \\
-0.458921 \\
\end{array}$ & $\begin{array}{r}-0.173954 \\
-1.398446 \\
\end{array}$ \\
\hline \multirow{11}{*}{$\begin{array}{l}\text { MN15-L/ } \\
\text { MG3S }\end{array}$} & $\mathrm{C}$ & 0.470651 & -0.149533 & 0.636042 \\
\hline & $\mathrm{O}$ & -0.696619 & -0.708910 & 0.656963 \\
\hline & $\mathrm{O}$ & -1.260299 & -0.567266 & -0.672666 \\
\hline & $\mathrm{H}$ & 0.827086 & 0.096725 & 1.651299 \\
\hline & $\mathrm{O}$ & -0.112384 & 1.520698 & -0.107068 \\
\hline & $\mathrm{H}$ & -0.766707 & 0.820017 & -0.588285 \\
\hline & $\mathrm{H}$ & -0.664108 & 1.941298 & 0.566072 \\
\hline & $\mathrm{C}$ & 1.504951 & -0.407591 & -0.416581 \\
\hline & $\mathrm{H}$ & 2.265869 & 0.382904 & -0.397256 \\
\hline & $\mathrm{H}$ & 1.989169 & -1.366120 & -0.157964 \\
\hline & $\mathrm{H}$ & 1.049496 & -0.488258 & -1.408463 \\
\hline \multirow{11}{*}{$\begin{array}{l}\text { MN15-L } \\
\text { /maug-TZ }\end{array}$} & $\mathrm{C}$ & 0.472382 & -0.159771 & 0.638310 \\
\hline & $\mathrm{O}$ & -0.696851 & -0.711423 & 0.655688 \\
\hline & $\mathrm{O}$ & -1.259944 & -0.568143 & -0.674859 \\
\hline & $\mathrm{H}$ & 0.829666 & 0.079841 & 1.653561 \\
\hline & $\mathrm{O}$ & -0.112019 & 1.528050 & -0.102727 \\
\hline & $\mathrm{H}$ & -0.763773 & 0.830040 & -0.582495 \\
\hline & $\mathrm{H}$ & -0.665230 & 1.953744 & 0.566876 \\
\hline & $\mathrm{C}$ & 1.503301 & -0.405742 & -0.419729 \\
\hline & $\mathrm{H}$ & 2.262500 & 0.386016 & -0.394712 \\
\hline & $\mathrm{H}$ & 1.989515 & -1.365654 & -0.169531 \\
\hline & $\mathrm{H}$ & 1.043738 & -0.478786 & -1.409999 \\
\hline \multirow{11}{*}{$\begin{array}{l}\text { M11-L/M } \\
\text { G3S }\end{array}$} & $\mathrm{C}$ & 0.465635 & -0.168508 & 0.630371 \\
\hline & $\mathrm{O}$ & -0.667705 & -0.723946 & 0.631176 \\
\hline & $\mathrm{O}$ & -1.203523 & -0.579059 & -0.668688 \\
\hline & $\mathrm{H}$ & 0.817784 & 0.050598 & 1.653036 \\
\hline & $\mathrm{O}$ & -0.159862 & 1.504191 & -0.088072 \\
\hline & $\mathrm{H}$ & -0.781558 & 0.792371 & -0.564866 \\
\hline & $\mathrm{H}$ & -0.717266 & 1.903573 & 0.575603 \\
\hline & $\mathrm{C}$ & 1.481499 & -0.349588 & -0.412445 \\
\hline & $\mathrm{H}$ & 2.193906 & 0.476984 & -0.399542 \\
\hline & $\mathrm{H}$ & 2.027734 & -1.272371 & -0.170949 \\
\hline & $\mathrm{H}$ & 1.025322 & -0.452073 & -1.396173 \\
\hline \multirow{9}{*}{$\begin{array}{l}\text { M11-L } \\
\text { /maug-TZ }\end{array}$} & $\mathrm{C}$ & 0.467989 & -0.168924 & 0.631494 \\
\hline & $\mathrm{O}$ & -0.663809 & -0.727876 & 0.634884 \\
\hline & $\mathrm{O}$ & -1.201934 & -0.587641 & -0.670893 \\
\hline & $\mathrm{H}$ & 0.820576 & 0.057485 & 1.650560 \\
\hline & $\mathrm{O}$ & -0.167867 & 1.509183 & -0.088231 \\
\hline & $\mathrm{H}$ & -0.784003 & 0.797799 & -0.562169 \\
\hline & $\mathrm{H}$ & -0.728505 & 1.910633 & 0.571894 \\
\hline & $\mathrm{C}$ & 1.483902 & -0.345060 & -0.414377 \\
\hline & $\mathrm{H}$ & 2.192243 & 0.483453 & -0.401350 \\
\hline
\end{tabular}




\begin{tabular}{|c|c|c|c|c|c|}
\hline & & $\begin{array}{l}\mathrm{H} \\
\mathrm{H}\end{array}$ & $\begin{array}{l}2.032328 \\
1.024889\end{array}$ & $\begin{array}{l}-1.265278 \\
-0.449524\end{array}$ & $\begin{array}{l}-0.172386 \\
-1.395326\end{array}$ \\
\hline \multirow[t]{42}{*}{ B2a-TS2 } & \multirow[t]{11}{*}{ QCISD/TZ } & $\mathrm{C}$ & 0.451445 & -0.217682 & 0.635638 \\
\hline & & $\mathrm{O}$ & -0.747883 & -0.694157 & 0.625098 \\
\hline & & $\mathrm{O}$ & -1.302695 & -0.443341 & -0.693717 \\
\hline & & $\mathrm{H}$ & 0.820899 & -0.052118 & 1.659779 \\
\hline & & $\mathrm{O}$ & -0.101335 & 1.543744 & 0.093745 \\
\hline & & $\mathrm{H}$ & -0.781047 & 0.930841 & -0.454953 \\
\hline & & $\mathrm{C}$ & 1.464339 & -0.493973 & -0.436920 \\
\hline & & $\mathrm{H}$ & 2.289299 & 0.230561 & -0.384672 \\
\hline & & $\mathrm{H}$ & 1.885991 & -1.493118 & -0.225434 \\
\hline & & $\mathrm{H}$ & 0.997563 & -0.505060 & -1.427287 \\
\hline & & $\mathrm{H}$ & 0.507901 & 1.908857 & -0.560747 \\
\hline & \multirow{11}{*}{$\begin{array}{l}\text { MN15-L/ } \\
\text { MG3S }\end{array}$} & $\mathrm{C}$ & 0.449618 & -0.209882 & 0.633709 \\
\hline & & $\mathrm{O}$ & -0.748260 & -0.694246 & 0.624402 \\
\hline & & $\mathrm{O}$ & -1.304611 & -0.438151 & -0.691951 \\
\hline & & $\mathrm{H}$ & 0.818657 & -0.042170 & 1.658902 \\
\hline & & $\mathrm{O}$ & -0.098494 & 1.536622 & 0.092398 \\
\hline & & $\mathrm{H}$ & -0.785610 & 0.925349 & -0.456120 \\
\hline & & $\mathrm{C}$ & 1.464885 & -0.495663 & -0.435037 \\
\hline & & $\mathrm{H}$ & 2.291440 & 0.227715 & -0.386679 \\
\hline & & $\mathrm{H}$ & 1.884866 & -1.494106 & -0.217753 \\
\hline & & $\mathrm{H}$ & 1.000931 & -0.512048 & -1.427034 \\
\hline & & $\mathrm{H}$ & 0.513627 & 1.894731 & -0.562143 \\
\hline & \multirow{11}{*}{$\begin{array}{l}\text { MN15-L } \\
\text { /maug-TZ }\end{array}$} & $\mathrm{C}$ & 0.451445 & -0.217682 & 0.635638 \\
\hline & & $\mathrm{O}$ & -0.747883 & -0.694157 & 0.625098 \\
\hline & & $\mathrm{O}$ & -1.302695 & -0.443341 & -0.693717 \\
\hline & & $\mathrm{H}$ & 0.820899 & -0.052118 & 1.659779 \\
\hline & & $\mathrm{O}$ & -0.101335 & 1.543744 & 0.093745 \\
\hline & & $\mathrm{H}$ & -0.781047 & 0.930841 & -0.454953 \\
\hline & & $\mathrm{C}$ & 1.464339 & -0.493973 & -0.436920 \\
\hline & & $\mathrm{H}$ & 2.289299 & 0.230561 & -0.384672 \\
\hline & & $\mathrm{H}$ & 1.885991 & -1.493118 & -0.225434 \\
\hline & & $\mathrm{H}$ & 0.997563 & -0.505060 & -1.427287 \\
\hline & & $\mathrm{H}$ & 0.507901 & 1.908857 & -0.560747 \\
\hline & \multirow{9}{*}{$\begin{array}{l}\text { M11-L/M } \\
\text { G3S }\end{array}$} & $\mathrm{C}$ & 0.447001 & -0.212677 & 0.627391 \\
\hline & & $\mathrm{O}$ & -0.713537 & -0.701730 & 0.608731 \\
\hline & & $\mathrm{O}$ & -1.240257 & -0.478918 & -0.685735 \\
\hline & & $\mathrm{H}$ & 0.809661 & -0.047346 & 1.655006 \\
\hline & & $\mathrm{O}$ & -0.165399 & 1.516871 & 0.095183 \\
\hline & & $\mathrm{H}$ & -0.800630 & 0.880482 & -0.457157 \\
\hline & & $\mathrm{C}$ & 1.449906 & -0.434405 & -0.425069 \\
\hline & & $\mathrm{H}$ & 2.242677 & 0.316493 & -0.376084 \\
\hline & & $\mathrm{H}$ & 1.917837 & -1.408954 & -0.224009 \\
\hline
\end{tabular}




\begin{tabular}{|c|c|c|c|c|c|}
\hline & & $\begin{array}{l}\mathrm{H} \\
\mathrm{H} \\
\end{array}$ & $\begin{array}{l}0.987995 \\
0.414566\end{array}$ & $\begin{array}{r}-0.466099 \\
1.918132 \\
\end{array}$ & $\begin{array}{c}-1.411764 \\
-0.545361 \\
\end{array}$ \\
\hline & \multirow{11}{*}{$\begin{array}{l}\text { M11-L } \\
\text { /maug-TZ }\end{array}$} & $\mathrm{C}$ & 0.450591 & -0.212087 & 0.628520 \\
\hline & & $\mathrm{O}$ & -0.707810 & -0.706792 & 0.612649 \\
\hline & & $\mathrm{O}$ & -1.237428 & -0.490973 & -0.687972 \\
\hline & & $\mathrm{H}$ & 0.813858 & -0.038725 & 1.652665 \\
\hline & & $\mathrm{O}$ & -0.178817 & 1.521447 & 0.094593 \\
\hline & & $\mathrm{H}$ & -0.804565 & 0.880681 & -0.456245 \\
\hline & & $\mathrm{C}$ & 1.454380 & -0.426548 & -0.426782 \\
\hline & & $\mathrm{H}$ & 2.240043 & 0.330199 & -0.379759 \\
\hline & & $\mathrm{H}$ & 1.928882 & -1.396317 & -0.223228 \\
\hline & & $\mathrm{H}$ & 0.989517 & -0.463900 & -1.410598 \\
\hline & & $\mathrm{H}$ & 0.394882 & 1.930420 & -0.547420 \\
\hline \multirow{22}{*}{$\begin{array}{l}\text { B2dh-TS } \\
1\end{array}$} & \multirow[t]{11}{*}{ QCISD/TZ } & $\mathrm{O}$ & -0.905279 & -0.886748 & -0.315890 \\
\hline & & $\mathrm{C}$ & -1.157024 & 0.374413 & -0.305746 \\
\hline & & $\mathrm{O}$ & 0.173955 & -1.310323 & 0.522085 \\
\hline & & $\mathrm{C}$ & -0.456724 & 1.35354500 & 0.402073 \\
\hline & & $\mathrm{H}$ & -0.845708 & 2.358126 & 0.310289 \\
\hline & & $\mathrm{H}$ & -0.138601 & 1.060903 & 1.400580 \\
\hline & & $\mathrm{H}$ & 0.750578 & 1.113442 & -0.093504 \\
\hline & & $\mathrm{H}$ & -1.928434 & 0.606854 & -1.035498 \\
\hline & & $\mathrm{O}$ & 1.802146 & 0.305631 & -0.223232 \\
\hline & & $\mathrm{H}$ & 1.137377 & -0.581448 & 0.095425 \\
\hline & & $\mathrm{H}$ & 2.140699 & 0.205895 & -1.118956 \\
\hline & \multirow{11}{*}{$\begin{array}{l}\text { MN15-L } \\
\text { /maug-TZ }\end{array}$} & $\mathrm{O}$ & -0.876649 & -0.895063 & -0.335598 \\
\hline & & $\mathrm{C}$ & -1.144686 & 0.369549 & -0.320941 \\
\hline & & $\mathrm{O}$ & 0.163126 & -1.292719 & 0.554043 \\
\hline & & $\mathrm{C}$ & -0.462259 & 1.348351 & 0.420012 \\
\hline & & $\mathrm{H}$ & -0.867240 & 2.359180 & 0.346541 \\
\hline & & $\mathrm{H}$ & -0.137108 & 1.024395 & 1.418291 \\
\hline & & $\mathrm{H}$ & 0.756581 & 1.103314 & -0.096331 \\
\hline & & $\mathrm{H}$ & -1.896883 & 0.603249 & -1.087987 \\
\hline & & $\mathrm{O}$ & 1.786349 & 0.309509 & -0.234609 \\
\hline & & $\mathrm{H}$ & 1.137189 & -0.567997 & 0.105815 \\
\hline & & $\mathrm{H}$ & 2.066521 & 0.196638 & -1.151441 \\
\hline \multirow{9}{*}{$\begin{array}{l}\text { B2dh-TS } \\
2\end{array}$} & \multirow[t]{9}{*}{ QCISD/TZ } & $\mathrm{O}$ & -0.931007 & -0.870211 & -0.302855 \\
\hline & & $\mathrm{C}$ & -1.145521 & 0.398521 & -0.298864 \\
\hline & & $\mathrm{O}$ & 0.153825 & -1.312415 & 0.522031 \\
\hline & & $\mathrm{C}$ & -0.424629 & 1.359175 & 0.415589 \\
\hline & & $\mathrm{H}$ & -0.790710 & 2.372254 & 0.318815 \\
\hline & & $\mathrm{H}$ & -0.139028 & 1.055087 & 1.420410 \\
\hline & & $\mathrm{H}$ & 0.776668 & 1.094824 & -0.084387 \\
\hline & & $\mathrm{H}$ & -1.907848 & 0.650754 & -1.031204 \\
\hline & & $\mathrm{O}$ & 1.752195 & 0.247602 & -0.403174 \\
\hline
\end{tabular}




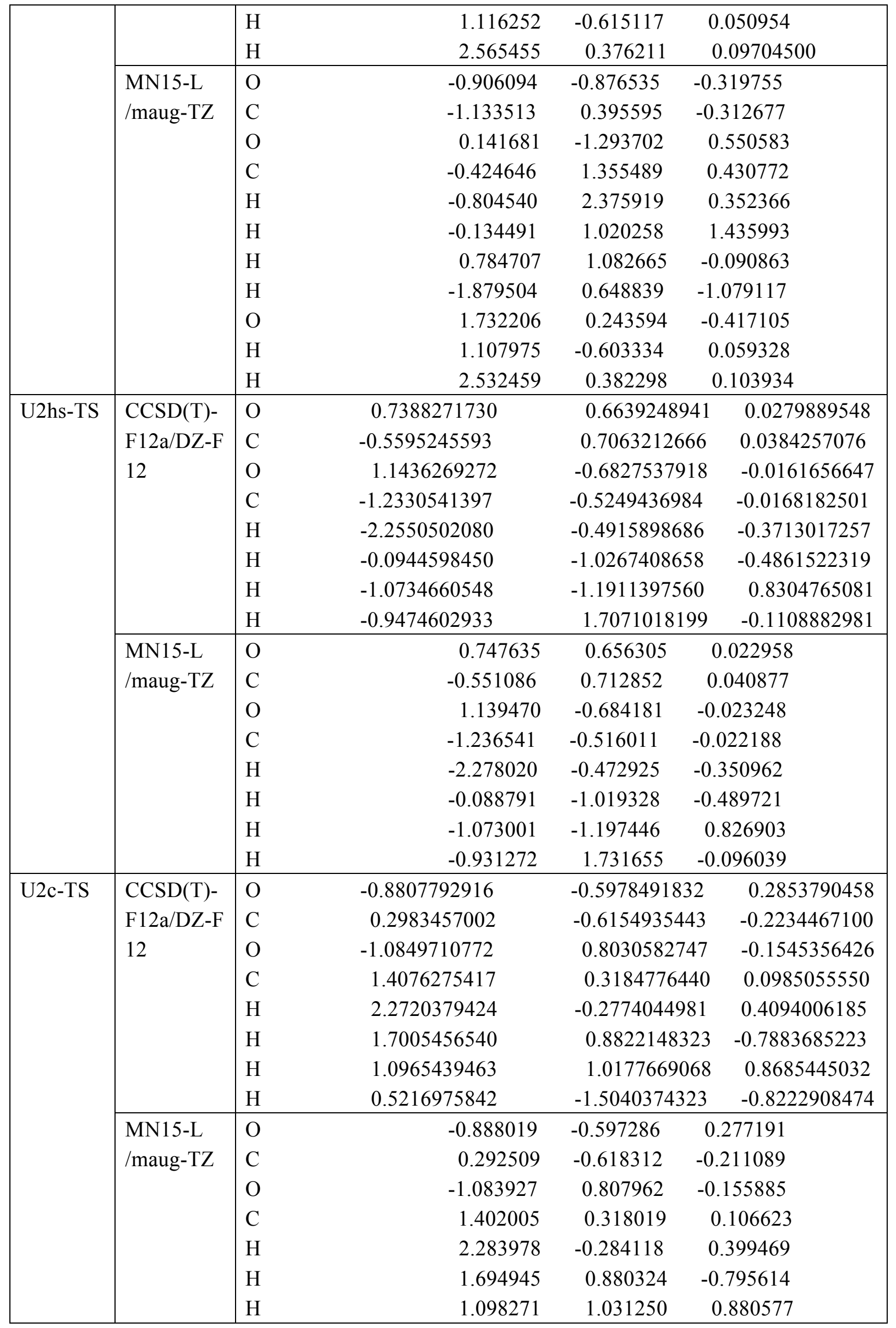




\begin{tabular}{|c|c|c|c|c|c|}
\hline & & $\mathrm{H}$ & 0.531286 & -1.511106 & -0.828084 \\
\hline \multirow{43}{*}{ B2a-TS1 } & \multirow[t]{11}{*}{ QCISD/TZ } & $\mathrm{O}$ & -1.566535 & -0.308404 & -0.461375 \\
\hline & & $\mathrm{O}$ & -0.862979 & 0.022525 & 0.744996 \\
\hline & & $\mathrm{C}$ & 0.514624 & 0.121255 & 0.443742 \\
\hline & & $\mathrm{H}$ & 0.940346 & 0.291534 & 1.436015 \\
\hline & & $\mathrm{O}$ & 0.760456 & 1.210798 & -0.409209 \\
\hline & & $\mathrm{H}$ & -1.519616 & 0.528733 & -0.938757 \\
\hline & & $\mathrm{H}$ & 0.577676 & 2.013866 & 0.083370 \\
\hline & & $\mathrm{C}$ & 1.092883 & -1.117555 & -0.197196 \\
\hline & & $\mathrm{H}$ & 2.174933 & -1.017999 & -0.261051 \\
\hline & & $\mathrm{H}$ & 0.845458 & -1.990919 & 0.403113 \\
\hline & & $\mathrm{H}$ & 0.688621 & -1.246763 & -1.197272 \\
\hline & \multirow{11}{*}{$\begin{array}{l}\text { MN15-L/ } \\
\text { MG3S }\end{array}$} & $\mathrm{O}$ & -1.569599 & -0.311306 & -0.460236 \\
\hline & & $\mathrm{O}$ & -0.867545 & 0.046614 & 0.744663 \\
\hline & & $\mathrm{C}$ & 0.514169 & 0.125845 & 0.449786 \\
\hline & & $\mathrm{H}$ & 0.934439 & 0.303703 & 1.462573 \\
\hline & & $\mathrm{O}$ & 0.777675 & 1.202432 & -0.418572 \\
\hline & & $\mathrm{H}$ & -1.502926 & 0.512777 & -0.971168 \\
\hline & & $\mathrm{H}$ & 0.577135 & 2.021147 & 0.051267 \\
\hline & & $\mathrm{C}$ & 1.084518 & -1.130728 & -0.188249 \\
\hline & & $\mathrm{H}$ & 2.181997 & -1.071632 & -0.202991 \\
\hline & & $\mathrm{H}$ & 0.772375 & -2.014898 & 0.384596 \\
\hline & & $\mathrm{H}$ & 0.720611 & -1.223717 & -1.220341 \\
\hline & \multirow{11}{*}{$\begin{array}{l}\text { MN15-L } \\
\text { /maug-TZ }\end{array}$} & $\mathrm{O}$ & -1.571643 & -0.310204 & -0.461409 \\
\hline & & $\mathrm{O}$ & -0.868298 & 0.045475 & 0.746005 \\
\hline & & $\mathrm{C}$ & 0.514287 & 0.124764 & 0.450595 \\
\hline & & $\mathrm{H}$ & 0.934883 & 0.302587 & 1.462004 \\
\hline & & $\mathrm{O}$ & 0.777977 & 1.203148 & -0.418110 \\
\hline & & $\mathrm{H}$ & -1.492270 & 0.512218 & -0.974620 \\
\hline & & $\mathrm{H}$ & 0.576580 & 2.021906 & 0.052980 \\
\hline & & $\mathrm{C}$ & 1.085468 & -1.130866 & -0.189074 \\
\hline & & $\mathrm{H}$ & 2.182318 & -1.064640 & -0.213804 \\
\hline & & $\mathrm{H}$ & 0.783713 & -2.014709 & 0.389689 \\
\hline & & $\mathrm{H}$ & 0.711967 & -1.228101 & -1.217268 \\
\hline & \multirow{10}{*}{$\begin{array}{l}\text { M11-L/M } \\
\text { G3S }\end{array}$} & $\mathrm{O}$ & -1.516125 & -0.339131 & -0.454872 \\
\hline & & $\mathrm{O}$ & -0.848361 & 0.004954 & 0.733340 \\
\hline & & $\mathrm{C}$ & 0.493566 & 0.136458 & 0.437161 \\
\hline & & $\mathrm{H}$ & 0.916918 & 0.317489 & 1.447911 \\
\hline & & $\mathrm{O}$ & 0.702653 & 1.202540 & -0.404262 \\
\hline & & $\mathrm{H}$ & -1.470493 & 0.485147 & -0.945030 \\
\hline & & $\mathrm{H}$ & 0.481478 & 2.000508 & 0.067698 \\
\hline & & $\mathrm{C}$ & 1.104148 & -1.072498 & -0.189783 \\
\hline & & $\mathrm{H}$ & 2.189021 & -0.952835 & -0.255075 \\
\hline & & $\mathrm{H}$ & 0.879802 & -1.961140 & 0.405670 \\
\hline
\end{tabular}




\begin{tabular}{|c|c|c|c|c|c|}
\hline & & $\mathrm{H}$ & 0.711654 & -1.219829 & -1.199085 \\
\hline & M11-L & $\mathrm{O}$ & -1.525926 & -0.332849 & -0.454443 \\
\hline & /maug-TZ & $\mathrm{O}$ & -0.850583 & 0.015007 & 0.733370 \\
\hline & & $\mathrm{C}$ & 0.495361 & 0.133967 & 0.436479 \\
\hline & & $\mathrm{H}$ & 0.918342 & 0.312641 & 1.446012 \\
\hline & & $\mathrm{O}$ & 0.719667 & 1.199893 & -0.406081 \\
\hline & & $\mathrm{H}$ & -1.482104 & 0.491073 & -0.945473 \\
\hline & & $\mathrm{H}$ & 0.500087 & 1.999272 & 0.065176 \\
\hline & & $\mathrm{C}$ & 1.098333 & -1.082948 & -0.188104 \\
\hline & & $\mathrm{H}$ & 2.182771 & -0.968247 & -0.254169 \\
\hline & & $\mathrm{H}$ & 0.869668 & -1.966857 & 0.411022 \\
\hline & & $\mathrm{H}$ & 0.703807 & -1.230400 & -1.195582 \\
\hline \multirow{32}{*}{ B2a-P2 } & \multirow[t]{11}{*}{ QCISD/TZ } & $\mathrm{C}$ & 0.512132 & 0.136744 & 0.470203 \\
\hline & & $\mathrm{O}$ & -0.853254 & -0.137924 & 0.757043 \\
\hline & & $\mathrm{O}$ & -1.525468 & -0.304905 & -0.512056 \\
\hline & & $\mathrm{H}$ & 0.921073 & 0.284464 & 1.486397 \\
\hline & & $\mathrm{O}$ & 0.663604 & 1.349418 & -0.212548 \\
\hline & & $\mathrm{H}$ & -2.000036 & 0.539588 & -0.560460 \\
\hline & & $\mathrm{C}$ & 1.188323 & -1.022711 & -0.255804 \\
\hline & & $\mathrm{H}$ & 2.264772 & -0.822351 & -0.357733 \\
\hline & & $\mathrm{H}$ & 1.044072 & -1.961328 & 0.298106 \\
\hline & & $\mathrm{H}$ & 0.749167 & -1.140244 & -1.259441 \\
\hline & & $\mathrm{H}$ & 0.539157 & 1.162956 & -1.152778 \\
\hline & \multirow{11}{*}{$\begin{array}{l}\text { MN15-L/ } \\
\text { MG3S }\end{array}$} & $\mathrm{C}$ & 0.511123 & 0.137633 & 0.469808 \\
\hline & & $\mathrm{O}$ & -0.851896 & -0.143525 & 0.755733 \\
\hline & & $\mathrm{O}$ & -1.524253 & -0.306852 & -0.511544 \\
\hline & & $\mathrm{H}$ & 0.919405 & 0.285023 & 1.487458 \\
\hline & & $\mathrm{O}$ & 0.656960 & 1.350669 & -0.210473 \\
\hline & & $\mathrm{H}$ & -1.997655 & 0.537551 & -0.559387 \\
\hline & & $\mathrm{C}$ & 1.192677 & -1.018040 & -0.256972 \\
\hline & & $\mathrm{H}$ & 2.269077 & -0.815406 & -0.355391 \\
\hline & & $\mathrm{H}$ & 1.049264 & -1.958790 & 0.293650 \\
\hline & & $\mathrm{H}$ & 0.757683 & -1.135160 & -1.262584 \\
\hline & & $\mathrm{H}$ & 0.532941 & 1.166881 & -1.150482 \\
\hline & \multirow{10}{*}{$\begin{array}{l}\text { MN15-L } \\
\text { /maug-TZ }\end{array}$} & $\mathrm{C}$ & 0.512132 & 0.136744 & 0.470203 \\
\hline & & $\mathrm{O}$ & -0.853254 & -0.137924 & 0.757043 \\
\hline & & $\mathrm{O}$ & -1.525468 & -0.304905 & -0.512056 \\
\hline & & $\mathrm{H}$ & 0.921073 & 0.284464 & 1.486397 \\
\hline & & $\mathrm{O}$ & 0.663604 & 1.349418 & -0.212548 \\
\hline & & $\mathrm{H}$ & -2.000036 & 0.539588 & -0.560460 \\
\hline & & $\mathrm{C}$ & 1.188323 & -1.022711 & -0.255804 \\
\hline & & $\mathrm{H}$ & 2.264772 & -0.822351 & -0.357733 \\
\hline & & $\mathrm{H}$ & 1.044072 & -1.961328 & 0.298106 \\
\hline & & $\mathrm{H}$ & 0.749167 & -1.140244 & -1.259441 \\
\hline
\end{tabular}




\begin{tabular}{|c|c|c|c|c|c|}
\hline & & $\mathrm{H}$ & 0.539157 & 1.162956 & -1.152778 \\
\hline & M11-L/M & $\mathrm{C}$ & 0.488365 & 0.142251 & 0.457935 \\
\hline & G3S & $\mathrm{O}$ & -0.832632 & -0.166442 & 0.738566 \\
\hline & & $\mathrm{O}$ & -1.478850 & -0.320335 & 0.503811 \\
\hline & & $\mathrm{H}$ & 0.897298 & 0.288900 & 1.474756 \\
\hline & & $\mathrm{O}$ & 0.599536 & 1.334901 & -0.196489 \\
\hline & & $\mathrm{H}$ & -1.935245 & 0.519242 & 0.564169 \\
\hline & & $\mathrm{C}$ & 1.193726 & -0.971994 & 0.252646 \\
\hline & & $\mathrm{H}$ & 2.257662 & -0.740945 & 0.361822 \\
\hline & & $\mathrm{H}$ & 1.092565 & -1.913088 & 0.295565 \\
\hline & & $\mathrm{H}$ & 0.765297 & -1.118149 & 1.251518 \\
\hline & & $\mathrm{H}$ & 0.525444 & 1.157501 & -1.130673 \\
\hline & M11-L & $\mathrm{C}$ & 0.490300 & 0.140222 & 0.457065 \\
\hline & /maug-TZ & $\mathrm{O}$ & -0.837900 & -0.147128 & 0.739922 \\
\hline & & $\mathrm{O}$ & -1.488707 & -0.314417 & 0.502867 \\
\hline & & $\mathrm{H}$ & 0.898872 & 0.285343 & 1.472344 \\
\hline & & $\mathrm{O}$ & 0.623860 & 1.331470 & -0.202438 \\
\hline & & $\mathrm{H}$ & -1.938721 & 0.527959 & 0.574008 \\
\hline & & $\mathrm{C}$ & 1.182359 & -0.989030 & 0.247669 \\
\hline & & $\mathrm{H}$ & 2.248976 & -0.773018 & 0.352077 \\
\hline & & $\mathrm{H}$ & 1.064933 & -1.925007 & 0.304057 \\
\hline & & $\mathrm{H}$ & 0.756147 & -1.131718 & 1.246955 \\
\hline & & $\mathrm{H}$ & 0.555810 & 1.149884 & -1.136664 \\
\hline \multirow{21}{*}{ U2hs-P } & CCSD(T)- & $\mathrm{O}$ & 0.5935393144 & -0.9182267788 & 0.4965725684 \\
\hline & $\mathrm{F} 12 \mathrm{a} / \mathrm{DZ}-\mathrm{F}$ & $\mathrm{C}$ & 1.2279157375 & 0.2953318342 & 0.4639430790 \\
\hline & 12 & $\mathrm{O}$ & -0.1578832596 & -1.1074120419 & -0.7222154610 \\
\hline & & $\mathrm{C}$ & 1.1867075776 & 1.2218609339 & -0.4905969195 \\
\hline & & $\mathrm{H}$ & 1.7592097519 & 2.1248338559 & -0.3442135291 \\
\hline & & $\mathrm{H}$ & 0.6174505848 & \multirow{2}{*}{$\begin{array}{l}1.0919754570 \\
03885354443\end{array}$} & $\begin{array}{ll}0 & -1.3963298977\end{array}$ \\
\hline & & $\mathrm{H}$ & 1.8030617183 & & $3 \quad 1.3766543303$ \\
\hline & & $\mathrm{H}$ & -1.0557124249 & -1.0605637045 & -0.3698911705 \\
\hline & QCISD/TZ & $\mathrm{O}$ & 0.6036892162 & -0.9320329313 & 0.4953760838 \\
\hline & & $\mathrm{C}$ & 1.2267545488 & 0.2863956696 & 0.4611901724 \\
\hline & & $\mathrm{O}$ & -0.1432990859 & -1.1361966608 & -0.7074268097 \\
\hline & & $\mathrm{C}$ & 1.1764679314 & 1.2138053756 & -0.4882854428 \\
\hline & & $\mathrm{H}$ & 1.7379558150 & 2.1225643246 & -0.3424297592 \\
\hline & & $\mathrm{H}$ & 0.6111018780 & 1.0821004736 & -1.3948207835 \\
\hline & & $\mathrm{H}$ & 1.8000373355 & & 1.3722498435 \\
\hline & & $\mathrm{H}$ & -1.0384186392 & -0.9890607809 & -0.3819303047 \\
\hline & \multirow{5}{*}{$\begin{array}{l}\text { MN15-L } \\
\text { /maug-TZ }\end{array}$} & $\mathrm{O}$ & 0.662483 & 0.709518 & -0.014075 \\
\hline & & $\mathrm{C}$ & -0.693934 & 0.564521 & 0.013269 \\
\hline & & $\mathrm{O}$ & 1.302144 & -0.572373 & -0.102736 \\
\hline & & $\mathrm{C}$ & -1.420401 & -0.556838 & 0.001457 \\
\hline & & $\mathrm{H}$ & -2.504946 & -0.466983 & 0.030669 \\
\hline
\end{tabular}




\begin{tabular}{|c|c|c|c|c|c|}
\hline & & $\begin{array}{l}\mathrm{H} \\
\mathrm{H} \\
\mathrm{H} \\
\end{array}$ & $\begin{array}{r}-0.971926 \\
-1.122930 \\
1.568793 \\
\end{array}$ & $\begin{array}{r}-1.546366 \\
1.571751 \\
-0.701667 \\
\end{array}$ & $\begin{array}{c}0.054953 \\
0.048155 \\
0.822262 \\
\end{array}$ \\
\hline \multirow[t]{16}{*}{$\mathrm{U} 2 \mathrm{c}-\mathrm{P}$} & \multirow{8}{*}{$\begin{array}{l}\mathrm{CCSD}(\mathrm{T})- \\
\mathrm{F} 12 \mathrm{a} / \mathrm{DZ}-\mathrm{F} \\
12\end{array}$} & $\mathrm{O}$ & 0.3524756342 & -0.8476895925 & $5 \quad 0.7580279552$ \\
\hline & & $\mathrm{C}$ & 0.3460744901 & 0.3191797985 & 0.0000000000 \\
\hline & & $\mathrm{O}$ & 0.3524756342 & -0.8476895925 & -0.7580279552 \\
\hline & & $\mathrm{C}$ & -0.9021575615 & 1.1417212585 & 0.0000000000 \\
\hline & & $\mathrm{H}$ & -0.9241232624 & 1.7805011546 & 0.8839362772 \\
\hline & & $\mathrm{H}$ & -0.9241232624 & 1.7805011546 & -0.8839362772 \\
\hline & & $\mathrm{H}$ & -1.7676991249 & 0.4827061159 & 0.0000000000 \\
\hline & & $\mathrm{H}$ & 1.2930564531 & 0.8581207031 & 0.0000000000 \\
\hline & \multirow{8}{*}{$\begin{array}{l}\text { MN15-L/ } \\
\text { maug-TZ }\end{array}$} & $\mathrm{O}$ & 0.352308 & -0.854687 & 0.742594 \\
\hline & & $\mathrm{C}$ & 0.352308 & 0.316014 & 0.000000 \\
\hline & & $\mathrm{O}$ & 0.352308 & -0.854687 & -0.742594 \\
\hline & & $\mathrm{C}$ & -0.903967 & 1.143640 & 0.000000 \\
\hline & & $\mathrm{H}$ & -0.931578 & 1.790904 & 0.890446 \\
\hline & & $\mathrm{H}$ & -0.931578 & 1.790904 & -0.890446 \\
\hline & & $\mathrm{H}$ & -1.776628 & 0.476021 & 0.000000 \\
\hline & & $\mathrm{H}$ & 1.312806 & 0.859242 & 0.000000 \\
\hline \multirow{25}{*}{$\begin{array}{l}\text { Anti- } \mathrm{CH}_{3} \\
\mathrm{CHOO}\end{array}$} & \multirow[t]{8}{*}{ QCISD/TZ } & $\mathrm{O}$ & 1.416511 & -1.190638 & 0.000000 \\
\hline & & $\mathrm{O}$ & 0.154823 & -0.686346 & 0.000000 \\
\hline & & $\mathrm{C}$ & 0.000000 & 0.566006 & 0.000000 \\
\hline & & $\mathrm{H}$ & 0.913357 & 1.178890 & 0.000000 \\
\hline & & $\mathrm{C}$ & -1.380794 & 1.108192 & 0.000000 \\
\hline & & $\mathrm{H}$ & -1.541518 & 1.749439 & 0.884132 \\
\hline & & $\mathrm{H}$ & -1.541518 & 1.749439 & 0.884132 \\
\hline & & $\mathrm{H}$ & -2.116232 & 0.292924 & 0.000000 \\
\hline & \multirow{8}{*}{$\begin{array}{l}\text { MN15-L/ } \\
\text { maug-TZ }\end{array}$} & $\mathrm{O}$ & 1.417481 & -1.191985 & 0.000000 \\
\hline & & $\mathrm{O}$ & 0.151924 & -0.687522 & 0.000000 \\
\hline & & $\mathrm{C}$ & 0.000000 & 0.565323 & 0.000000 \\
\hline & & $\mathrm{H}$ & 0.913682 & 1.175685 & 0.000000 \\
\hline & & $\mathrm{C}$ & -1.379594 & 1.111059 & 0.000000 \\
\hline & & $\mathrm{H}$ & -1.537478 & 1.752408 & 0.884401 \\
\hline & & $\mathrm{H}$ & -1.537478 & 1.752408 & 0.884401 \\
\hline & & $\mathrm{H}$ & -2.116407 & 0.297260 & 0.000000 \\
\hline & \multirow{8}{*}{$\begin{array}{l}\text { M11-L } \\
\text { /maug-TZ }\end{array}$} & $\mathrm{O}$ & 1.391520 & -1.155687 & 0.000000 \\
\hline & & $\mathrm{O}$ & 0.156322 & -0.678365 & 0.000000 \\
\hline & & $\mathrm{C}$ & 0.000000 & 0.551690 & 0.000000 \\
\hline & & $\mathrm{H}$ & 0.908696 & 1.165498 & 0.000000 \\
\hline & & $\mathrm{C}$ & -1.357821 & 1.081023 & 0.000000 \\
\hline & & $\mathrm{H}$ & -1.526761 & 1.719270 & 0.876469 \\
\hline & & $\mathrm{H}$ & -1.526761 & 1.719270 & 0.876469 \\
\hline & & $\mathrm{H}$ & -2.090984 & 0.272096 & 0.000000 \\
\hline & QCISD/TZ & $\mathrm{O}$ & -0.937139 & 1.456331 & 0.098962 \\
\hline
\end{tabular}




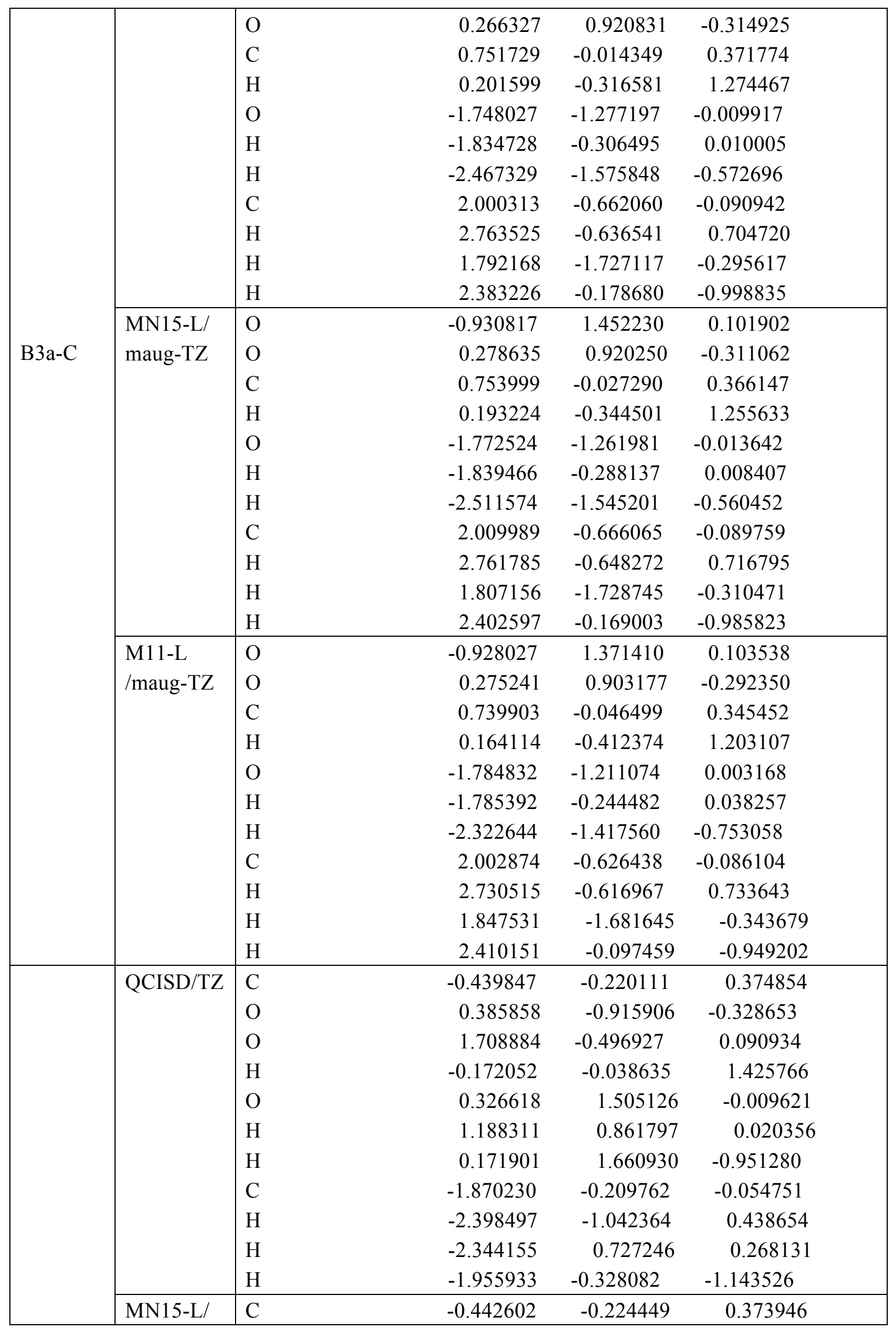




\begin{tabular}{|c|c|c|c|c|c|}
\hline \multirow{21}{*}{ B3a-TS1 } & maug-TZ & $\mathrm{O}$ & 0.381500 & -0.916500 & -0.332025 \\
\hline & & $\mathrm{O}$ & 1.707348 & -0.508321 & 0.092858 \\
\hline & & $\mathrm{H}$ & -0.171286 & -0.039434 & 1.421969 \\
\hline & & $\mathrm{O}$ & 0.335588 & 1.512949 & -0.008217 \\
\hline & & $\mathrm{H}$ & 1.189067 & 0.868093 & 0.020173 \\
\hline & & $\mathrm{H}$ & 0.186200 & 1.678629 & -0.949678 \\
\hline & & $\mathrm{C}$ & -1.873099 & -0.206559 & -0.054194 \\
\hline & & $\mathrm{H}$ & -2.406824 & -1.030816 & 0.447311 \\
\hline & & $\mathrm{H}$ & -2.338302 & 0.736837 & 0.262605 \\
\hline & & $\mathrm{H}$ & -1.960140 & -0.332283 & -1.141817 \\
\hline & M11-L & $\mathrm{C}$ & -0.448088 & -0.218257 & 0.358978 \\
\hline & /maug-TZ & $\mathrm{O}$ & 0.344892 & -0.907839 & -0.330100 \\
\hline & & $\mathrm{O}$ & 1.647948 & -0.549376 & 0.096177 \\
\hline & & $\mathrm{H}$ & -0.165084 & -0.017348 & 1.399324 \\
\hline & & $\mathrm{O}$ & 0.400673 & 1.492477 & -0.005920 \\
\hline & & $\mathrm{H}$ & 1.207493 & 0.806511 & 0.022605 \\
\hline & & $\mathrm{H}$ & 0.286024 & 1.691947 & -0.932563 \\
\hline & & $\mathrm{C}$ & -1.854123 & -0.164612 & -0.051968 \\
\hline & & $\mathrm{H}$ & -2.417474 & -0.933697 & 0.488734 \\
\hline & & $\mathrm{H}$ & -2.280794 & 0.801860 & 0.223357 \\
\hline & & $\mathrm{H}$ & -1.964995 & -0.334151 & -1.124773 \\
\hline \multirow{23}{*}{ B3a-TS2 } & QCISD/TZ & $\mathrm{C}$ & -0.447588 & -0.210314 & 0.378821 \\
\hline & & $\mathrm{O}$ & 0.371056 & -0.936066 & -0.296915 \\
\hline & & $\mathrm{O}$ & 1.700736 & -0.515693 & 0.100857 \\
\hline & & $\mathrm{H}$ & -0.173426 & 0.003481 & 1.421889 \\
\hline & & $\mathrm{O}$ & 0.329495 & 1.466081 & -0.212060 \\
\hline & & $\mathrm{H}$ & 1.190965 & 0.825673 & -0.122876 \\
\hline & & $\mathrm{C}$ & -1.873950 & -0.196932 & -0.059574 \\
\hline & & $\mathrm{H}$ & -2.405807 & -1.032724 & 0.424906 \\
\hline & & $\mathrm{H}$ & -2.351111 & 0.739817 & 0.258057 \\
\hline & & $\mathrm{H}$ & -1.940669 & -0.302802 & -1.149689 \\
\hline & & $\mathrm{H}$ & 0.398974 & 2.095458 & 0.517174 \\
\hline & MN15-L/ & $\mathrm{C}$ & -0.447588 & -0.210314 & 0.378821 \\
\hline & maug-TZ & $\mathrm{O}$ & 0.371056 & -0.936066 & -0.296915 \\
\hline & & $\mathrm{O}$ & 1.700736 & -0.515693 & 0.100857 \\
\hline & & $\mathrm{H}$ & -0.173426 & 0.003481 & 1.421889 \\
\hline & & $\mathrm{O}$ & 0.329495 & 1.466081 & -0.212060 \\
\hline & & $\mathrm{H}$ & 1.190965 & 0.825673 & -0.122876 \\
\hline & & $\mathrm{C}$ & -1.873950 & -0.196932 & -0.059574 \\
\hline & & $\mathrm{H}$ & -2.405807 & -1.032724 & 0.424906 \\
\hline & & $\mathrm{H}$ & -2.351111 & 0.739817 & 0.258057 \\
\hline & & $\mathrm{H}$ & -1.940669 & -0.302802 & -1.149689 \\
\hline & & $\mathrm{H}$ & 0.398974 & 2.095458 & 0.517174 \\
\hline & M11-L & $\mathrm{C}$ & -0.450305 & -0.210599 & 0.367016 \\
\hline
\end{tabular}




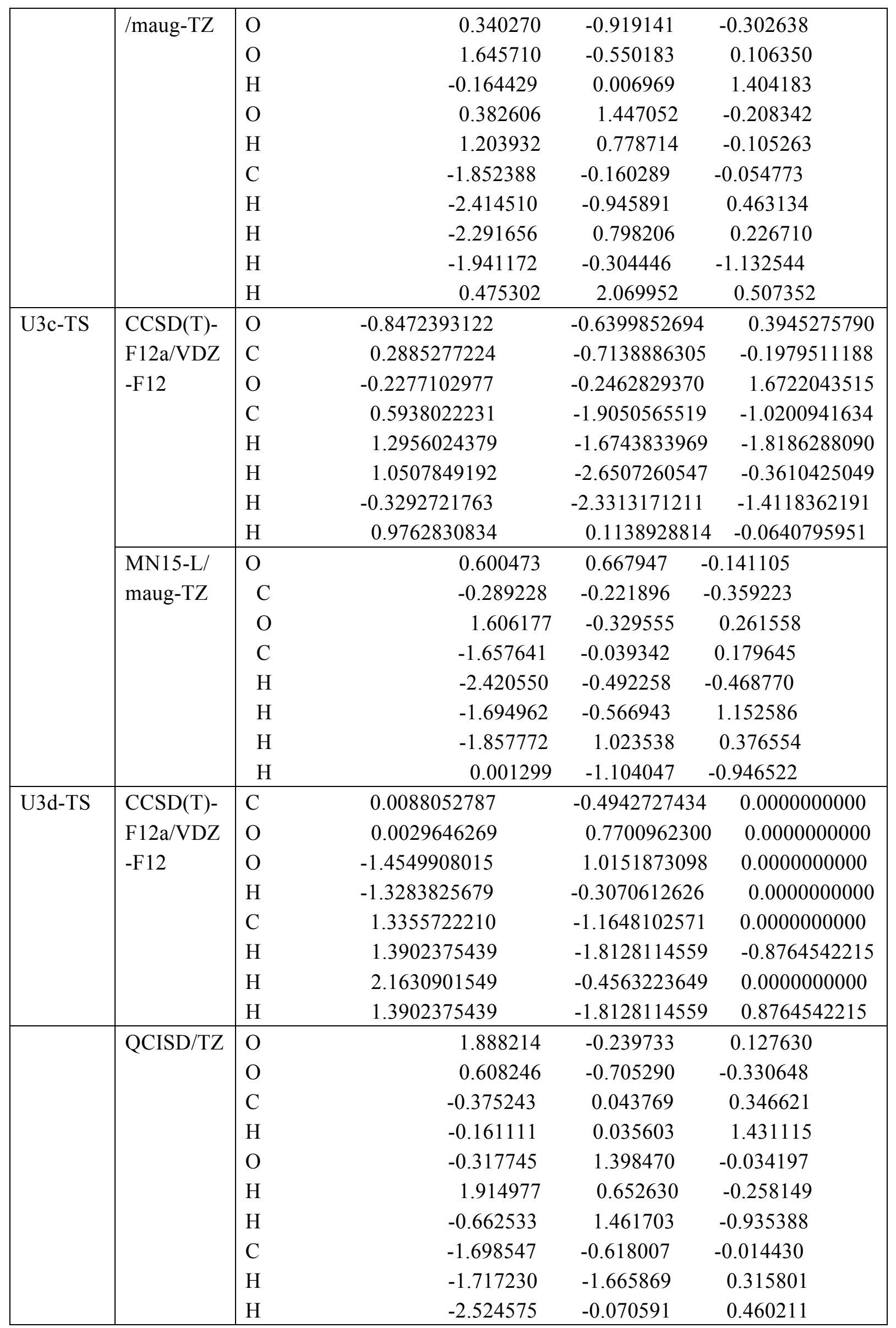




\begin{tabular}{|c|c|c|c|c|c|}
\hline \multirow{23}{*}{ B3a-P1 } & & $\mathrm{H}$ & -1.836511 & -0.595624 & -1.109021 \\
\hline & \multirow{11}{*}{$\begin{array}{l}\text { MN15-L/ } \\
\text { maug-TZ }\end{array}$} & $\mathrm{O}$ & 1.889966 & -0.239440 & 0.126649 \\
\hline & & $\mathrm{O}$ & 0.607964 & -0.707114 & -0.331078 \\
\hline & & $\mathrm{C}$ & -0.375275 & 0.043207 & 0.346854 \\
\hline & & $\mathrm{H}$ & -0.160996 & 0.035709 & 1.430275 \\
\hline & & $\mathrm{O}$ & -0.317101 & 1.399146 & -0.034967 \\
\hline & & $\mathrm{H}$ & 1.905842 & 0.659073 & -0.247210 \\
\hline & & $\mathrm{H}$ & -0.664373 & 1.460767 & -0.936200 \\
\hline & & $\mathrm{C}$ & -1.699438 & -0.617673 & -0.013769 \\
\hline & & $\mathrm{H}$ & -1.718561 & -1.664750 & 0.318673 \\
\hline & & $\mathrm{H}$ & -2.524667 & -0.068094 & 0.459646 \\
\hline & & $\mathrm{H}$ & -1.835600 & -0.596647 & -1.108525 \\
\hline & \multirow{11}{*}{$\begin{array}{l}\text { M11-L } \\
\text { /maug-TZ }\end{array}$} & $\mathrm{O}$ & 1.839933 & -0.247801 & 0.121480 \\
\hline & & $\mathrm{O}$ & 0.588028 & -0.709155 & -0.322191 \\
\hline & & $\mathrm{C}$ & -0.363448 & 0.041581 & 0.331174 \\
\hline & & $\mathrm{H}$ & -0.161422 & 0.028295 & 1.416126 \\
\hline & & $\mathrm{O}$ & -0.269884 & 1.368307 & -0.033508 \\
\hline & & $\mathrm{H}$ & 1.836300 & 0.652742 & -0.215279 \\
\hline & & $\mathrm{H}$ & -0.608200 & 1.448324 & -0.923810 \\
\hline & & $\mathrm{C}$ & -1.682148 & -0.578305 & -0.012419 \\
\hline & & $\mathrm{H}$ & -1.727379 & -1.618833 & 0.317196 \\
\hline & & $\mathrm{H}$ & -2.490612 & -0.020418 & 0.464836 \\
\hline & & $\mathrm{H}$ & -1.839726 & -0.560582 & -1.097846 \\
\hline \multirow{21}{*}{ B3a-P2 } & \multirow[t]{11}{*}{ QCISD/TZ } & $\mathrm{C}$ & -0.403524 & 0.068207 & 0.334606 \\
\hline & & $\mathrm{O}$ & 0.588325 & -0.757272 & -0.252946 \\
\hline & & $\mathrm{O}$ & 1.859010 & -0.200202 & 0.156949 \\
\hline & & $\mathrm{H}$ & -0.226193 & 0.085902 & 1.430136 \\
\hline & & $\mathrm{O}$ & -0.378705 & 1.367344 & -0.188017 \\
\hline & & $\mathrm{H}$ & 2.196949 & 0.100146 & -0.700917 \\
\hline & & $\mathrm{C}$ & -1.725217 & -0.581088 & -0.039566 \\
\hline & & $\mathrm{H}$ & -1.756972 & -1.621210 & 0.311745 \\
\hline & & $\mathrm{H}$ & -2.549137 & -0.014560 & 0.414965 \\
\hline & & $\mathrm{H}$ & -1.838803 & -0.559819 & -1.133754 \\
\hline & & $\mathrm{H}$ & 0.397568 & 1.807867 & 0.179697 \\
\hline & \multirow{10}{*}{$\begin{array}{l}\text { MN15-L/ } \\
\text { maug-TZ }\end{array}$} & $\mathrm{C}$ & -0.403524 & 0.068207 & 0.334606 \\
\hline & & $\mathrm{O}$ & 0.588325 & -0.757272 & -0.252946 \\
\hline & & $\mathrm{O}$ & 1.859010 & -0.200202 & 0.156949 \\
\hline & & $\mathrm{H}$ & -0.226193 & 0.085902 & 1.430136 \\
\hline & & $\mathrm{O}$ & -0.378705 & 1.367344 & -0.188017 \\
\hline & & $\mathrm{H}$ & 2.196949 & 0.100146 & -0.700917 \\
\hline & & $\mathrm{C}$ & -1.725217 & -0.581088 & -0.039566 \\
\hline & & $\mathrm{H}$ & -1.756972 & -1.621210 & 0.311745 \\
\hline & & $\mathrm{H}$ & -2.549137 & -0.014560 & 0.414965 \\
\hline & & $\mathrm{H}$ & -1.838803 & -0.559819 & -1.133754 \\
\hline
\end{tabular}




\begin{tabular}{|l|l|lcc|}
\hline & $\mathrm{H}$ & 0.397568 & 1.807867 & 0.179697 \\
\cline { 2 - 5 } /m11-L & $\mathrm{C}$ & -0.391953 & 0.069380 & 0.320060 \\
& $\mathrm{O}$ & 0.572386 & -0.750613 & -0.244601 \\
& $\mathrm{O}$ & 1.812260 & -0.203219 & 0.154316 \\
& $\mathrm{H}$ & -0.229389 & 0.078515 & 1.418182 \\
& $\mathrm{O}$ & -0.346963 & 1.340125 & -0.183206 \\
& $\mathrm{H}$ & 2.156634 & 0.078290 & -0.693358 \\
& $\mathrm{C}$ & -1.701724 & -0.552222 & -0.039101 \\
& $\mathrm{H}$ & -1.751079 & -1.584647 & 0.312331 \\
& $\mathrm{H}$ & -2.515024 & 0.017201 & 0.414643 \\
& $\mathrm{H}$ & -1.832706 & -0.538438 & -1.125220 \\
& $\mathrm{H}$ & 0.432155 & 1.755787 & 0.175600 \\
\hline
\end{tabular}


Table S18. Absolute energies in hartrees.

\begin{tabular}{|c|c|c|}
\hline Species & Methods & Total energies (a.u.) \\
\hline \multirow{13}{*}{$\mathrm{CH}_{2} \mathrm{OO}$} & W3X-L//CCSD(T)-F12a/TZ-F12 & -189.7224988 \\
\hline & W3X-L//CCSD(T)-F12a/DZ-F12 & -189.7224926 \\
\hline & W3X-L//QCISD/TZ & -189.7224524 \\
\hline & W3X//QCISD/TZ & -189.6753924 \\
\hline & MN15-L/MG3S & -189.475030345 \\
\hline & W2X//CCSD(T)-F12a/TZ-F12 & -189.7195457 \\
\hline & W2X//CCSD(T)-F12a/DZ-F12 & -189.7195236 \\
\hline & W2X//QCISD/TZ & -189.7194129 \\
\hline & MN15-L/maug-TZ & -189.473510010 \\
\hline & M11-L/MG3S & -189.582946853 \\
\hline & M11-L/maug-TZ & -189.586730567 \\
\hline & M06-L/MG3S & -189.624798767 \\
\hline & M06-L/maug-TZ & -189.624897938 \\
\hline \multirow{12}{*}{$\mathrm{H}_{2} \mathrm{O}$} & W3X-L//CCSD(T)-F12a/TZ-F12 & -76.49435433 \\
\hline & W3X-L//CCSD(T)-F12a/DZ-F12 & -76.49435416 \\
\hline & W3X-L//QCISD/TZ & -76.49434734 \\
\hline & W3X//QCISD/TZ & -76.47726156 \\
\hline & MN15-L/MG3S & -76.3773267850 \\
\hline & W2X//CCSD(T)-F12a/TZ-F12 & -76.49387421 \\
\hline & $\mathrm{W} 2 \mathrm{X} / / \mathrm{CCSD}(\mathrm{T})-\mathrm{F} 12 \mathrm{a} / \mathrm{DZ}-\mathrm{F} 12$ & -76.49387402 \\
\hline & MN15-L/maug-TZ & -76.3762370085 \\
\hline & M11-L/MG3S & -76.4360386824 \\
\hline & M11-L/maug-TZ & -76.4380432610 \\
\hline & M06-L/MG3S & -76.4453803828 \\
\hline & M06-L/maug-TZ & -76.4440340049 \\
\hline \multirow{10}{*}{ B1a-C } & W3X-L//CCSD(T)-F12a/TZ-F12 & -266.2303898 \\
\hline & W3X-L//CCSD(T)-F12a/DZ-F12 & -266.2303852 \\
\hline & W3X-L//QCISD/TZ & -266.2302388 \\
\hline & W3X//QCISD/TZ & -266.1661517 \\
\hline & MN15-L/MG3S & -265.866637392 \\
\hline & $\mathrm{W} 2 \mathrm{X} / / \mathrm{CCSD}(\mathrm{T})-\mathrm{F} 12 \mathrm{a} / \mathrm{TZ}-\mathrm{F} 12$ & -266.2272115 \\
\hline & W2X//CCSD(T)-F12a/DZ-F12 & -266.2271885 \\
\hline & MN15-L/maug-TZ & -265.863599333 \\
\hline & M11-L/MG3S & -266.031392656 \\
\hline & M11-L/maug-TZ & -266.036316978 \\
\hline \multirow{6}{*}{ B1dh-C } & W3X-L//CCSD(T)-F12a/TZ-F12 & -266.2304334 \\
\hline & W3X-L//CCSD(T)-F12a/DZ-F12 & -266.2304202 \\
\hline & W3X-L//QCISD/TZ & -266.2301566 \\
\hline & W3X//QCISD/TZ & -266.1660835 \\
\hline & W2X//CCSD(T)-F12a/TZ-F12 & -266.2271406 \\
\hline & W2X//CCSD(T)-F12a/DZ-F12 & -266.2271105 \\
\hline
\end{tabular}




\begin{tabular}{|c|c|c|}
\hline \multirow{12}{*}{ B1a-TS1 } & W3X-L//CCSD(T)-F12a/TZ-F12 & -266.2160648 \\
\hline & W3X-L//CCSD(T)-F12a/DZ-F12 & -266.2160557 \\
\hline & W3X-L//QCISD/TZ & -266.2160233 \\
\hline & W3X//QCISD/TZ & -266.1519570 \\
\hline & MN15-L/MG3S & -265.851536783 \\
\hline & $\mathrm{W} 2 \mathrm{X} / / \mathrm{CCSD}(\mathrm{T})-\mathrm{F} 12 \mathrm{a} / \mathrm{TZ}-\mathrm{F} 12$ & -266.2134758 \\
\hline & W2X//CCSD(T)-F12a/DZ-F12 & -266.2134599 \\
\hline & MN15-L/maug-TZ & -265.849908813 \\
\hline & M11-L/MG3S & -266.018740324 \\
\hline & M11-L/maug-TZ & -266.024797648 \\
\hline & M06-L/MG3S & -266.068948715 \\
\hline & M06-L/maug-TZ & -266.068508150 \\
\hline \multirow{12}{*}{ B1a-TS2 } & W3X-L//CCSD(T)-F12a/TZ-F12 & -266.2145389 \\
\hline & W3X-L//CCSD(T)-F12a/DZ-F12 & -266.2145345 \\
\hline & W3X-L//QCISD/TZ & -266.2144910 \\
\hline & W3X//QCISD/TZ & -266.1504765 \\
\hline & MN15-L/MG3S & -265.849934126 \\
\hline & $\mathrm{W} 2 \mathrm{X} / / \mathrm{CCSD}(\mathrm{T})-\mathrm{F} 12 \mathrm{a} / \mathrm{TZ}-\mathrm{F} 12$ & -266.2119755 \\
\hline & W2X//CCSD(T)-F12a/DZ-F12 & -266.2119597 \\
\hline & MN15-L/maug-TZ & -265.848479223 \\
\hline & M11-L/MG3S & -266.017302936 \\
\hline & M11-L/maug-TZ & -266.023621312 \\
\hline & M06-L/MG3S & -266.067500196 \\
\hline & M06-L/maug-TZ & -266.067243181 \\
\hline B1dh-TS & W3X-L//QCISD/TZ & -266.1933941 \\
\hline \multirow{7}{*}{ U1c-TS } & W3X-L//CCSD(T)-F12a/TZ-F12 & -189.6906267 \\
\hline & W3X-L//CCSD(T)-F12a/DZ-F12 & -189.6905779 \\
\hline & W3X-L//QCISD/TZ & -189.6903418 \\
\hline & W2X//QCISD/TZ & -189.6872413 \\
\hline & $\mathrm{W} 2 \mathrm{X} / / \mathrm{CCSD}(\mathrm{T})-\mathrm{F} 12 \mathrm{a} / \mathrm{DZ}-\mathrm{F} 12$ & -189.6871975 \\
\hline & $\mathrm{W} 2 \mathrm{X} / / \mathrm{CCSD}(\mathrm{T})-\mathrm{F} 12 \mathrm{a} / \mathrm{TZ}-\mathrm{F} 12$ & -189.6872339 \\
\hline & MN15-L/maug-TZ & -189.440156612 \\
\hline \multirow{7}{*}{ U1d-TS } & W3X-L//CCSD(T)-F12a/TZ-F12 & -189.6674254 \\
\hline & W3X-L//CCSD(T)-F12a/DZ-F12 & -189.6674215 \\
\hline & W3X-L//QCISD/TZ & -189.6673168 \\
\hline & W2X//QCISD/TZ & -189.6650027 \\
\hline & $\mathrm{W} 2 \mathrm{X} / / \mathrm{CCSD}(\mathrm{T})-\mathrm{F} 12 \mathrm{a} / \mathrm{DZ}-\mathrm{F} 12$ & -189.6651253 \\
\hline & $\mathrm{W} 2 \mathrm{X} / / \mathrm{CCSD}(\mathrm{T})-\mathrm{F} 12 \mathrm{a} / \mathrm{TZ}-\mathrm{F} 12$ & -189.6651394 \\
\hline & MN15-L/maug-TZ & -189.418805454 \\
\hline \multirow[b]{4}{*}{ B1a-P1 } & W3X-L//CCSD(T)-F12a/TZ-F12 & -266.2908271 \\
\hline & W3X-L//CCSD(T)-F12a/DZ-F12 & -266.2908148 \\
\hline & W3X-L//QCISD/TZ & -266.2907903 \\
\hline & W3X//QCISD/TZ & -266.2267089 \\
\hline
\end{tabular}




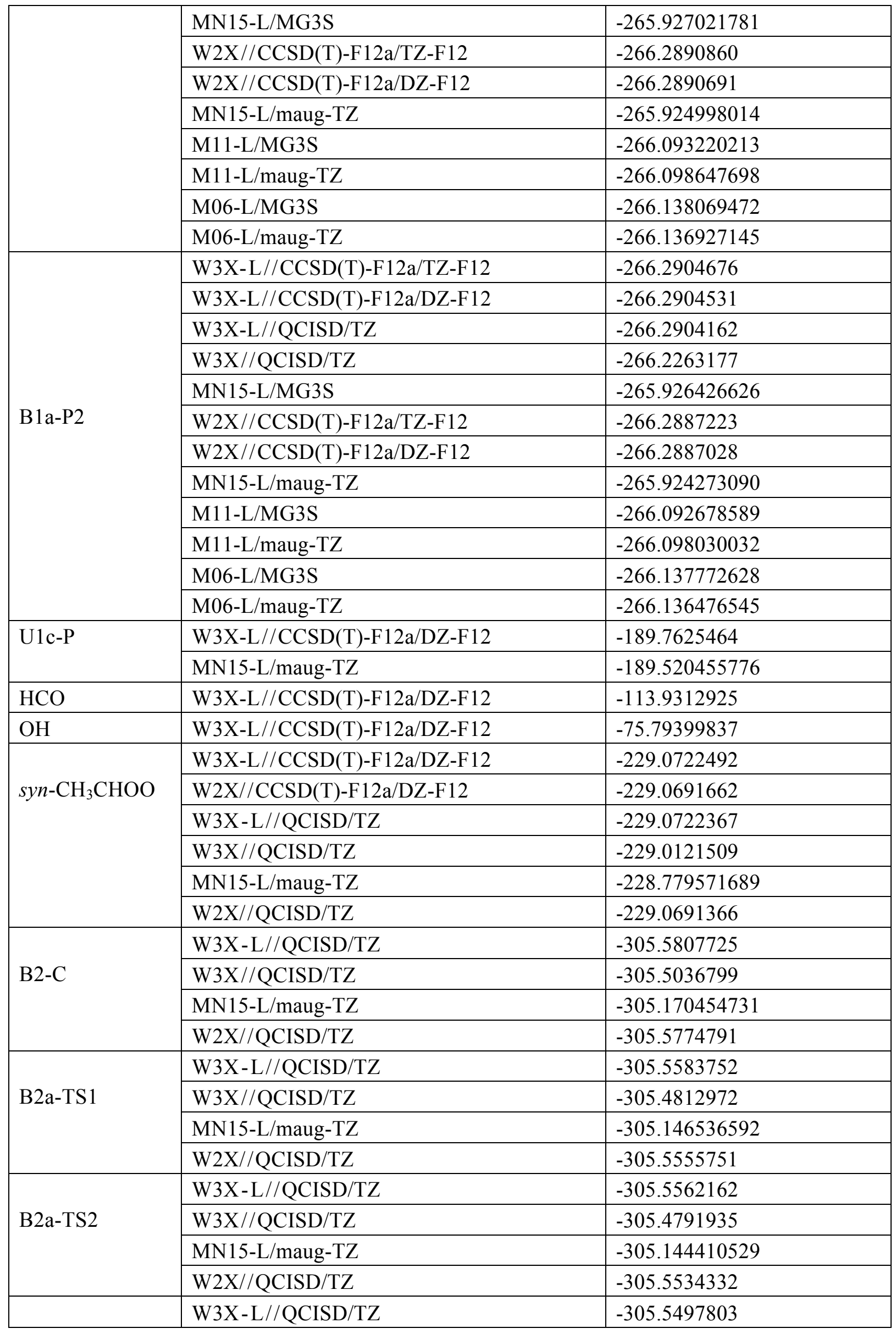




\begin{tabular}{|c|c|c|}
\hline \multirow[t]{3}{*}{ B2dh-TS1 } & W3X//QCISD/TZ & -305.4732431 \\
\hline & MN15-L/maug-TZ & -305.136164230 \\
\hline & W2X//QCISD/TZ & -305.5469789 \\
\hline \multirow{4}{*}{ B2dh-TS2 } & W3X-L//QCISD/TZ & -305.5494070 \\
\hline & W3X//QCISD/TZ & -305.4728800 \\
\hline & MN15-L/maug-TZ & -305.135920955 \\
\hline & W2X//QCISD/TZ & -305.5465973 \\
\hline \multirow{3}{*}{ U2hs-TS } & W3X-L//CCSD(T)-F12a/DZ-F12 & -229.0416554 \\
\hline & $\mathrm{W} 2 \mathrm{X} / / \mathrm{CCSD}(\mathrm{T})-\mathrm{F} 12 \mathrm{a} / \mathrm{DZ}-\mathrm{F} 12$ & -229.0392824 \\
\hline & MN15-L/maug-TZ & -305.079953737 \\
\hline \multirow{3}{*}{ U2c-TS } & W3X-L//CCSD(T)-F12a/DZ-F12 & -229.0329562 \\
\hline & $\mathrm{W} 2 \mathrm{X} / / \mathrm{CCSD}(\mathrm{T})-\mathrm{F} 12 \mathrm{a} / \mathrm{DZ}-\mathrm{F} 12$ & -229.0293185 \\
\hline & MN15-L/maug-TZ & -305.114477293 \\
\hline \multirow{4}{*}{ B2a-P1 } & W3X-L//QCISD/TZ & -305.6301135 \\
\hline & W3X//QCISD/TZ & -305.5530710 \\
\hline & MN15-L/maug-TZ & -305.217791858 \\
\hline & $\mathrm{W} 2 \mathrm{X} / / \mathrm{QCISD} / \mathrm{TZ}$ & -305.6280981 \\
\hline \multirow{4}{*}{ B2a-P2 } & W3X-L//QCISD/TZ & -305.6294277 \\
\hline & W3X//QCISD/TZ & -305.5523824 \\
\hline & MN15-L/maug-TZ & -305.217056189 \\
\hline & W2X//QCISD/TZ & -305.6274075 \\
\hline \multirow{4}{*}{ U2hs-P } & W3X-L//CCSD(T)-F12a/DZ-F12 & -229.1015335 \\
\hline & W3X-L//QCISD/TZ & -229.1015078 \\
\hline & W2X//CCSD(T)-F12a/DZ-F12 & -229.0996078 \\
\hline & MN15-L/maug-TZ & -228.805971444 \\
\hline \multirow{6}{*}{ anti- $\mathrm{CH}_{3} \mathrm{CHO}$} & W3X-L//CCSD(T)-F12a/DZ-F12 & -229.0665186 \\
\hline & $\mathrm{W} 2 \mathrm{X} / / \mathrm{CCSD}(\mathrm{T})-\mathrm{F} 12 \mathrm{a} / \mathrm{DZ}-\mathrm{F} 12$ & -229.0632449 \\
\hline & W3X-L//QCISD/TZ & -229.0665013 \\
\hline & W3X//QCISD/TZ & -229.0063006 \\
\hline & MN15-L/maug-TZ & -228.773029053 \\
\hline & W2X//QCISD/TZ & -229.0632163 \\
\hline \multirow{4}{*}{ B3a-C } & W3X-L//QCISD/TZ & -305.5769097 \\
\hline & $\mathrm{W} 3 \mathrm{X} / / \mathrm{QCISD} / \mathrm{TZ}$ & -305.4997153 \\
\hline & MN15-L/maug-TZ & -305.165241777 \\
\hline & W2X//QCISD/TZ & -305.5736406 \\
\hline \multirow{4}{*}{ B3a-TS 1} & W3X-L//QCISD/TZ & -305.5631035 \\
\hline & W3X//QCISD/TZ & -305.4860399 \\
\hline & MN15-L/maug-TZ & -305.150037735 \\
\hline & $\mathrm{W} 2 \mathrm{X} / / \mathrm{QCISD} / \mathrm{TZ}$ & -305.5603391 \\
\hline \multirow{4}{*}{ B3a-TS2 } & W3X-L//QCISD/TZ & -305.5619116 \\
\hline & W3X//QCISD/TZ & -305.4848887 \\
\hline & MN15-L/maug-TZ & -305.148959794 \\
\hline & W2X//QCISD/TZ & -305.5591595 \\
\hline
\end{tabular}




\begin{tabular}{|l|l|l|}
\hline \multirow{4}{*}{ U3c-TS } & W3X-L//CCSD(T)-F12a/DZ-F12 & -229.0399721 \\
\cline { 2 - 3 } & W2X//CCSD(T)-F12a/DZ-F12 & -229.0363308 \\
\cline { 2 - 3 } & MN15-L/maug-TZ & -228.745685462 \\
\hline \multirow{5}{*}{ B3d-TS } & W3X-L//CCSD(T)-F12a/DZ-F12 & -229.0131086 \\
\cline { 2 - 3 } & W2X//CCSD(T)-F12a/DZ-F12 & -229.0155786 \\
\cline { 2 - 3 } & MN15-L/maug-TZ & -228.722019016 \\
\hline \multirow{5}{*}{ B3a-P2 } & W3X-L//QCISD/TZ & -305.6318875 \\
\cline { 2 - 3 } & W3X//QCISD/TZ & -305.5548703 \\
\cline { 2 - 3 } & MN15-L/maug-TZ & -305.219241949 \\
\cline { 2 - 3 } & W2X//QCISD/TZ & -305.6298781 \\
\hline & W3X-L//QCISD/TZ & -305.6318741 \\
\cline { 2 - 3 } & W3X//QCISD/TZ & -305.5548247 \\
\cline { 2 - 3 } & MN15-L/maug-TZ & -305.218711192 \\
\cline { 2 - 3 } & W2X//QCISD/TZ & -305.6298609 \\
\hline U3c-P & W3X-L//CCSD(T)-F12a/DZ-F12 & -229.1075209 \\
\cline { 2 - 3 } & MN15-L/maug-TZ & -228.818495027 \\
\hline CH ${ }_{3}$ CO & W3X-L//CCSD(T)-F12a/DZ-F12 & -229.0026865 \\
\hline TSR & W3X-L//QCISD/TZ & -305.212581478 \\
\hline TS2R & MN15-L/maug-TZ & -305.202313518 \\
\hline TS3R & MN15-L/maug-TZ & -305.214525137 \\
\hline TS4R & MN15-L/maug-TZ & \\
\hline
\end{tabular}




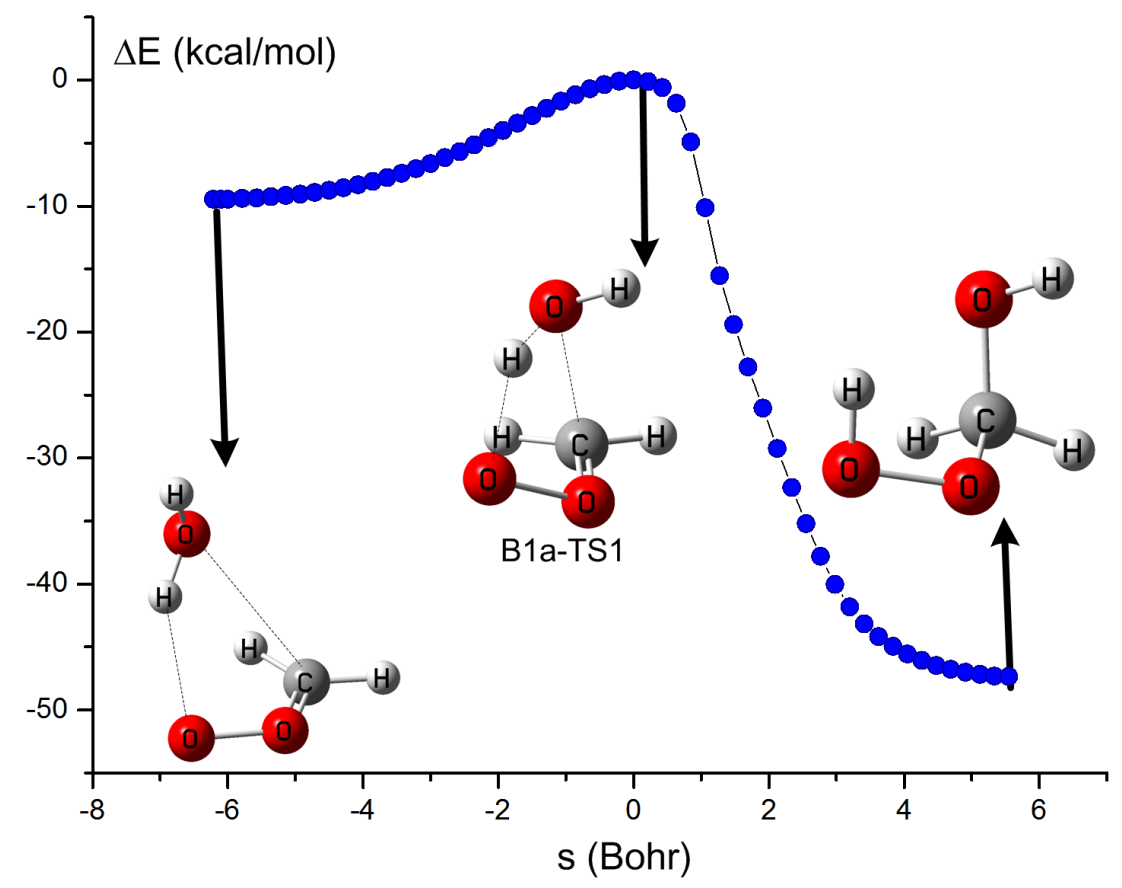

Figure S1. Potential energy profile along minimum energy path through B1a-TS1 calculated by MN15-L/MG3S.

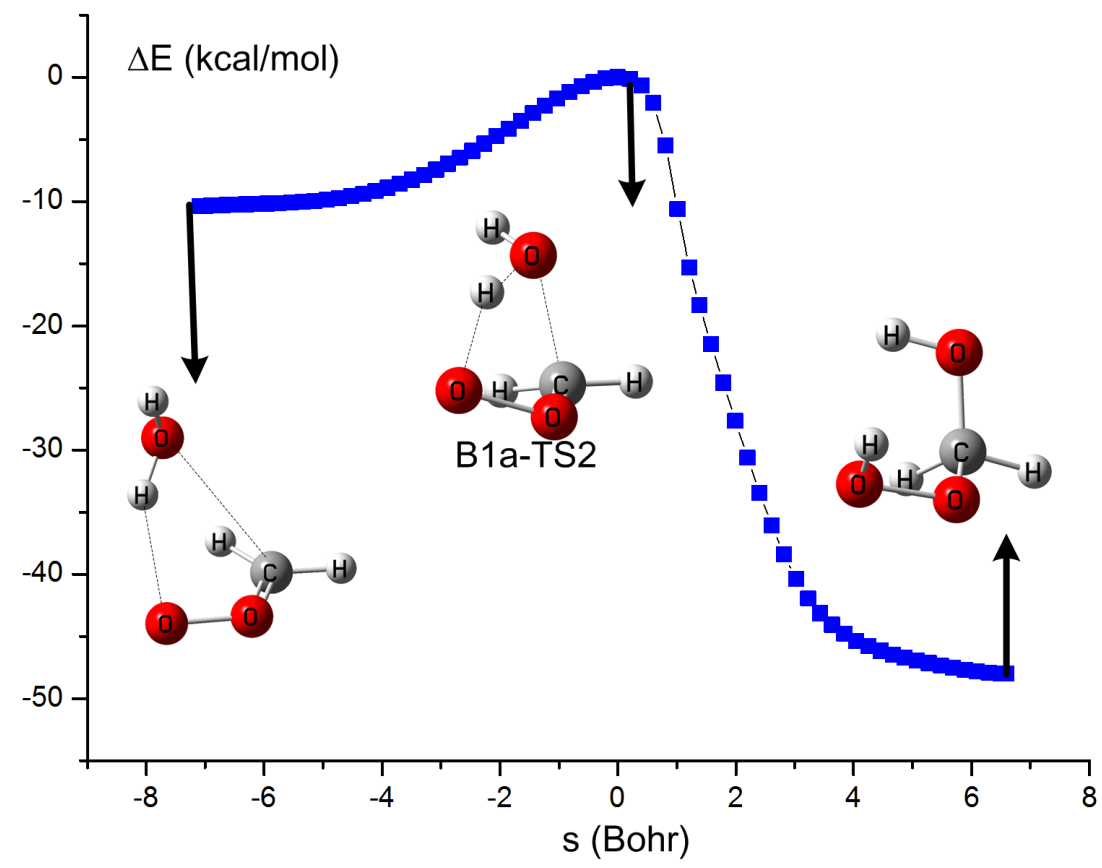

Figure S2. Potential energy profile along minimum energy path through B1a-TS2 calculated by MN15-L/MG3S. 


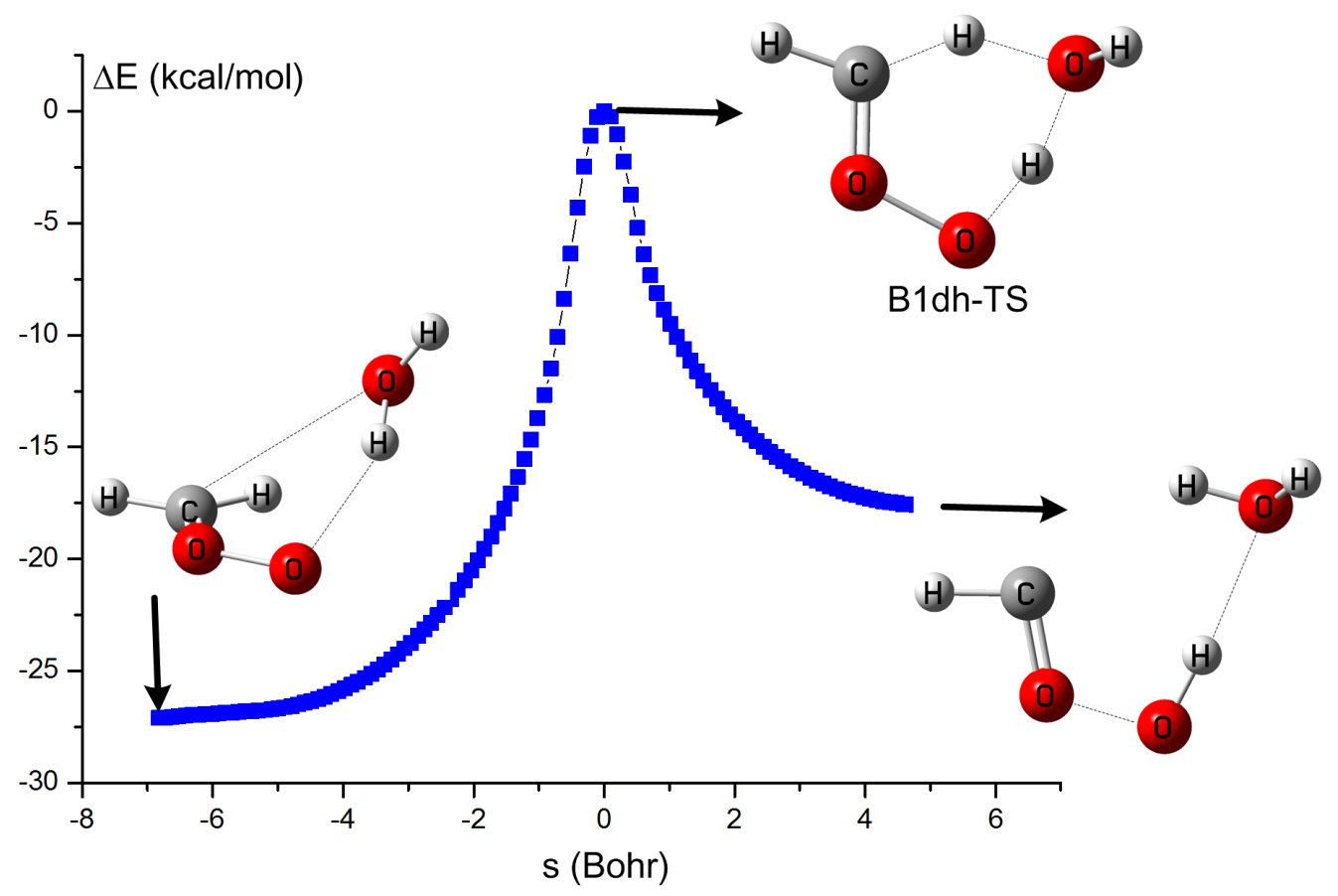

Figure S3. Potential energy profile along minimum energy path through B1dh-TS calculated by MN15-L/MG3S.

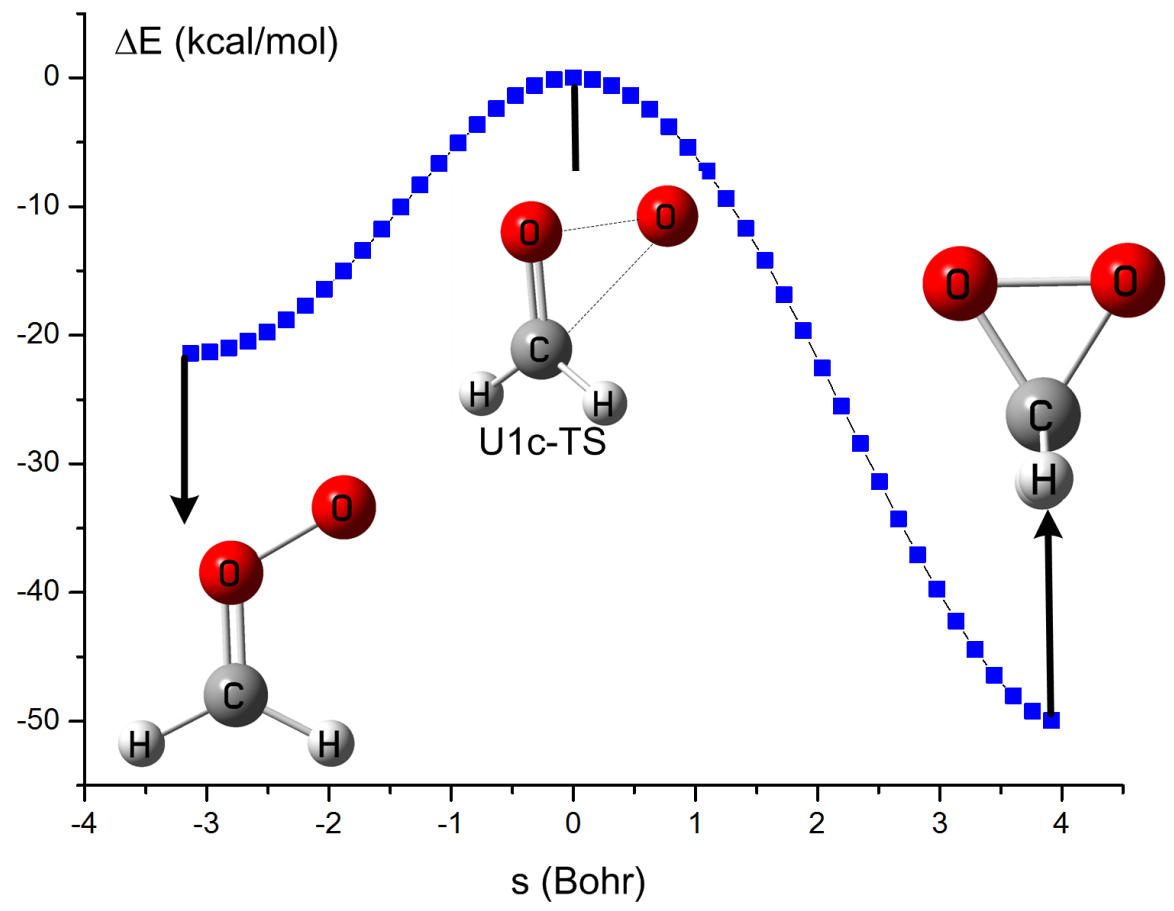

Figure S4. Potential energy profile along minimum energy path through U1c-TS calculated by MN15-L/MG3S. 


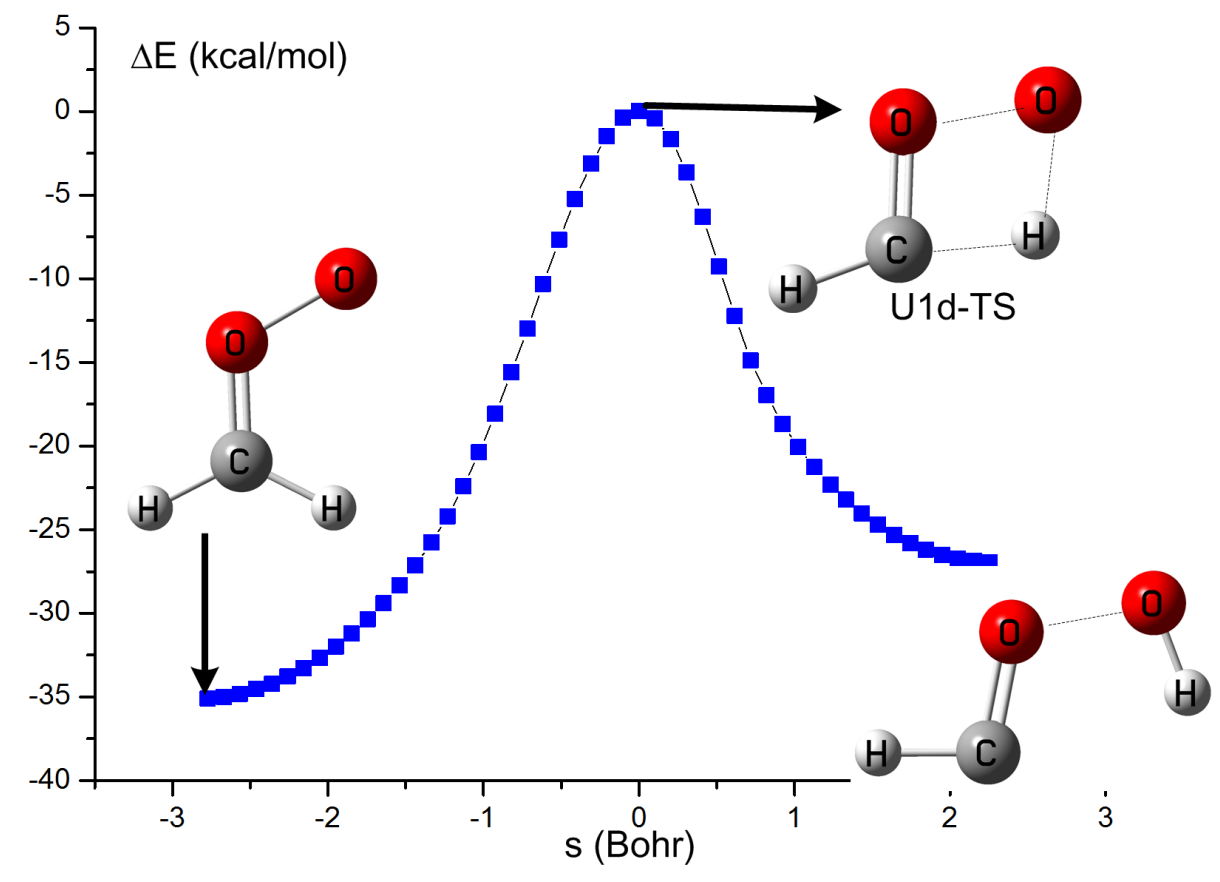

Figure S5. Potential energy profile along minimum energy path through U1d-TS calculated by MN15-L/MG3S.

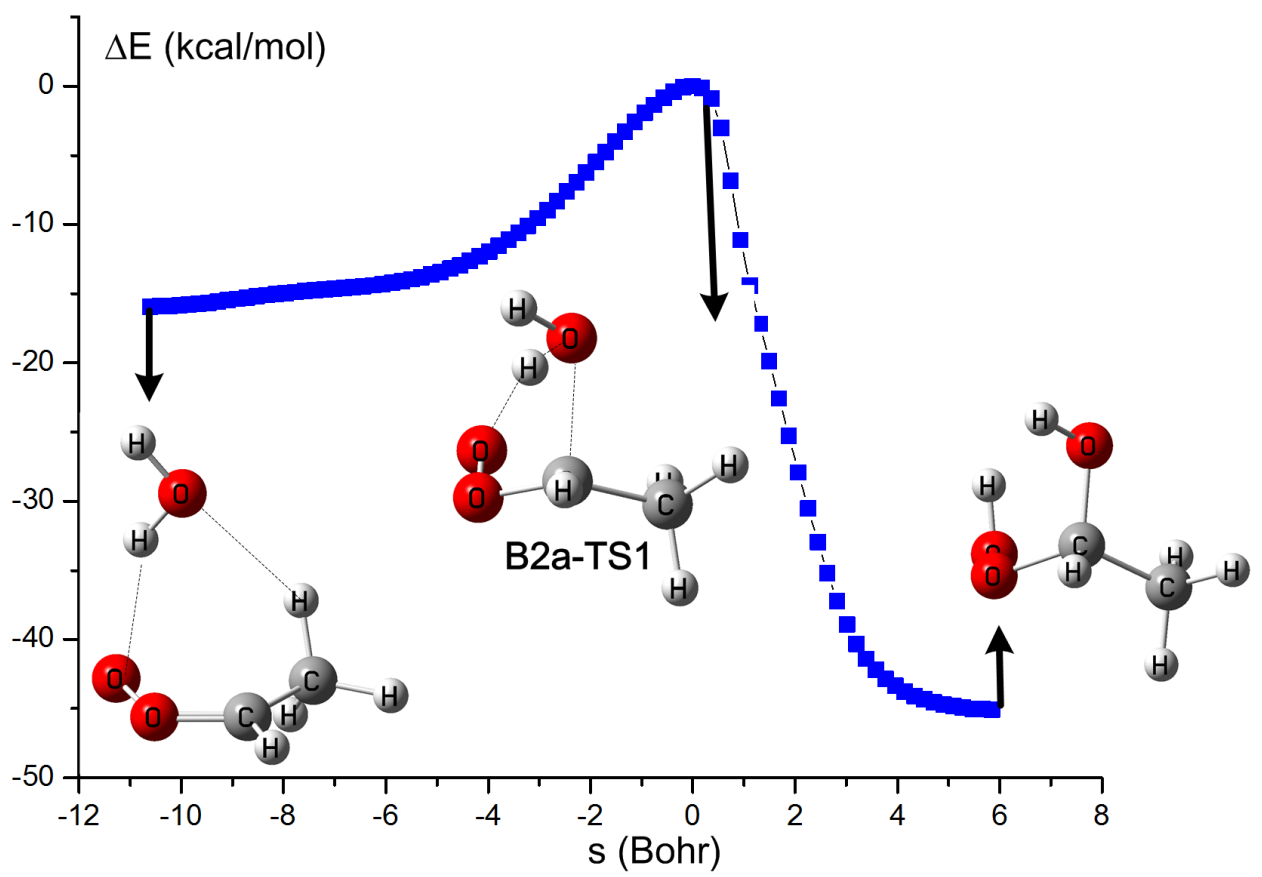

Figure S6. Potential energy profile along minimum energy path through B2a-TS1 calculated by MN15-L/MG3S. 


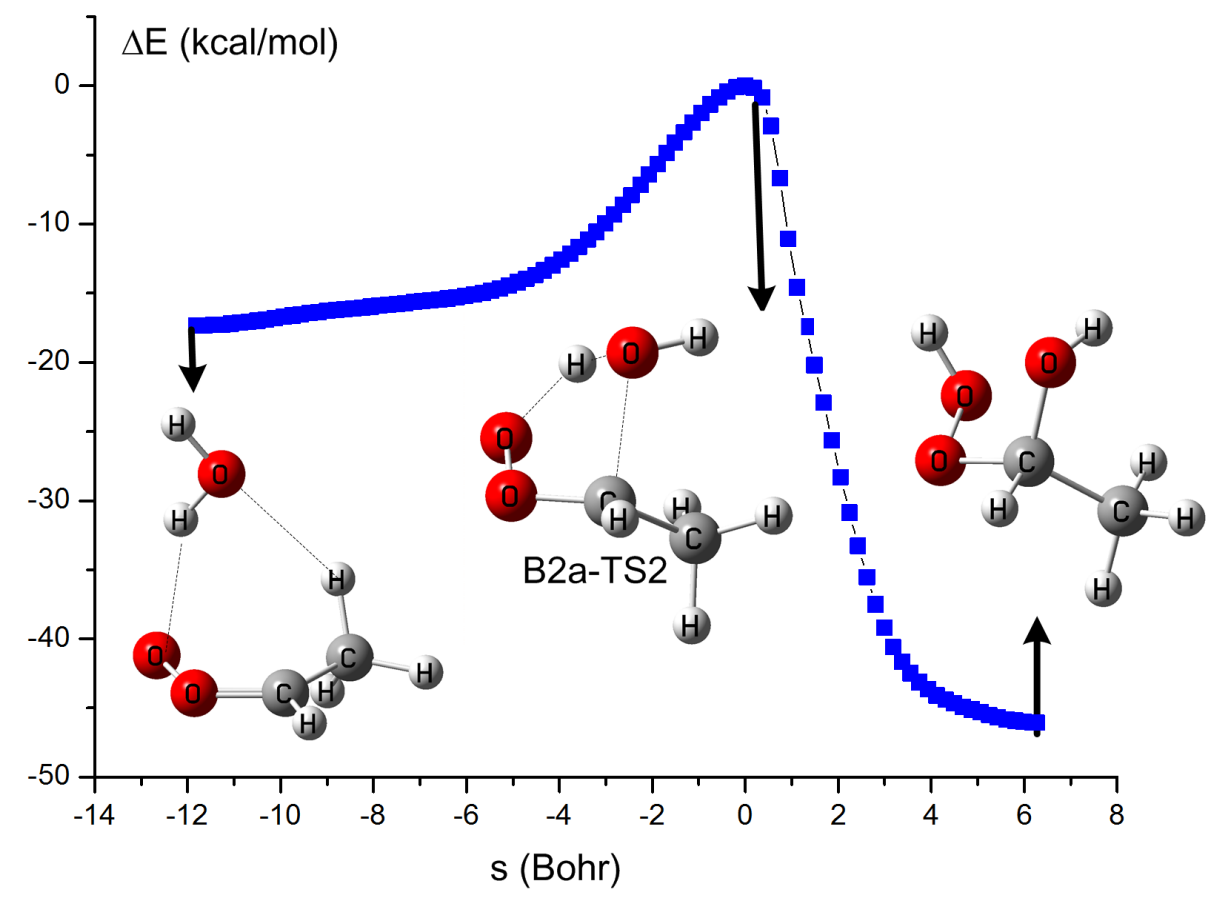

Figure S7. Potential energy profile along minimum energy path through B2a-TS2 calculated by MN15-L/MG3S.

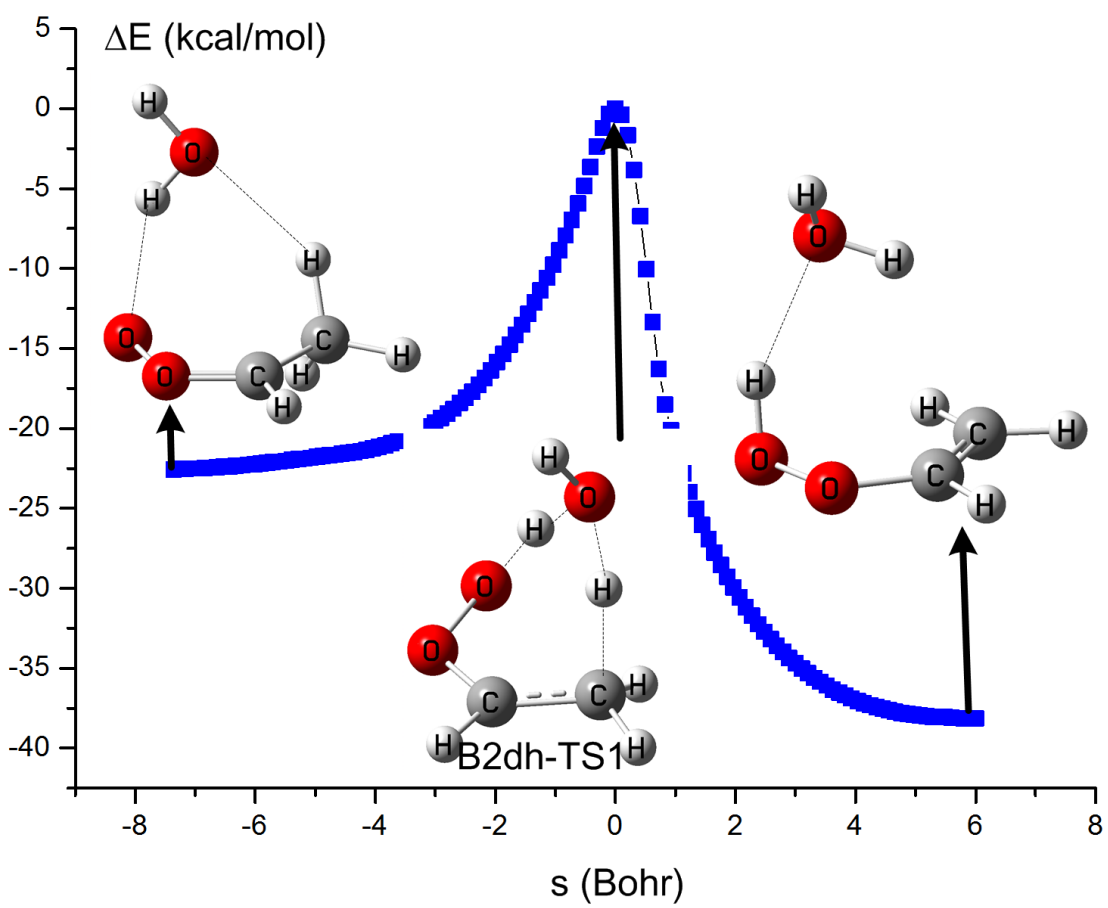

Figure S8. Potential energy profile along minimum energy path through B2dh-TS1 calculated by MN15-L/MG3S. 


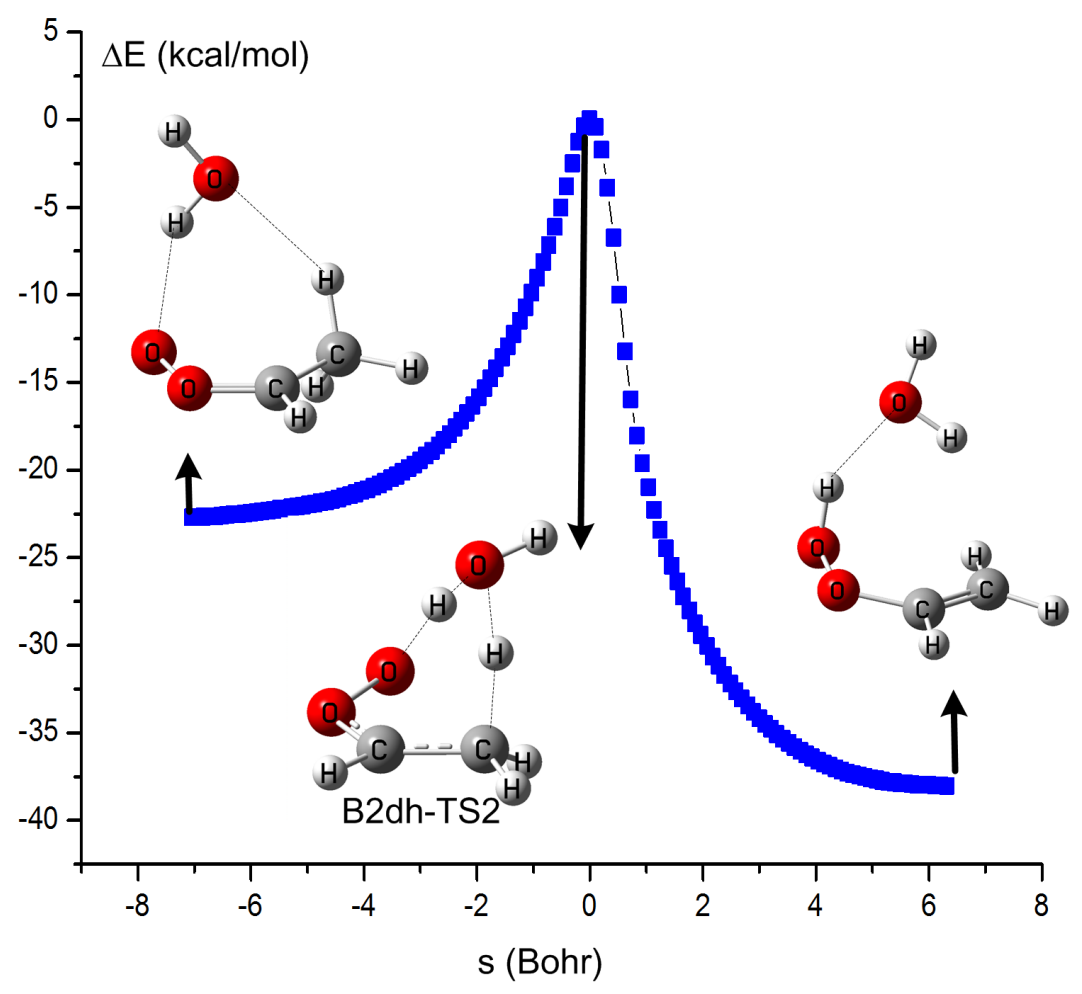

Figure S9. Potential energy profile along minimum energy path through B2dh-TS2 calculated by MN15-L/MG3S.

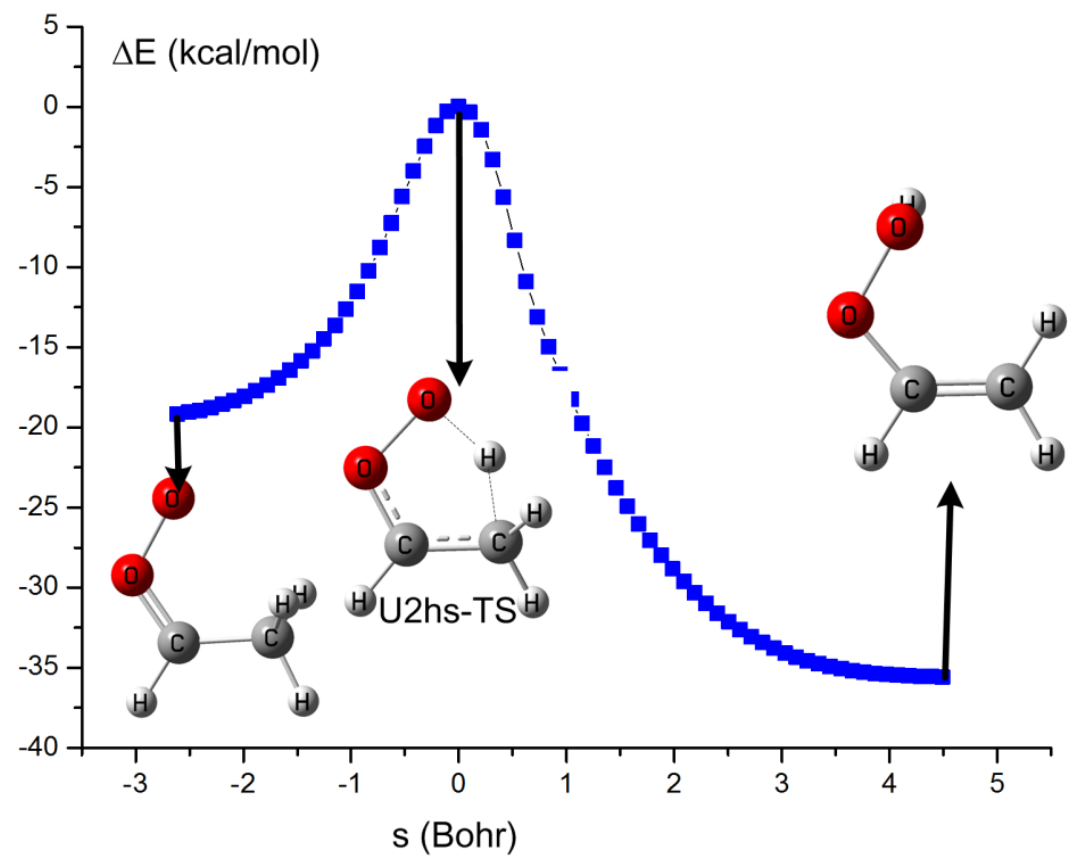

Figure S10. Potential energy profile along minimum energy path through U2hs-TS calculated by 
MN15-L/MG3S.

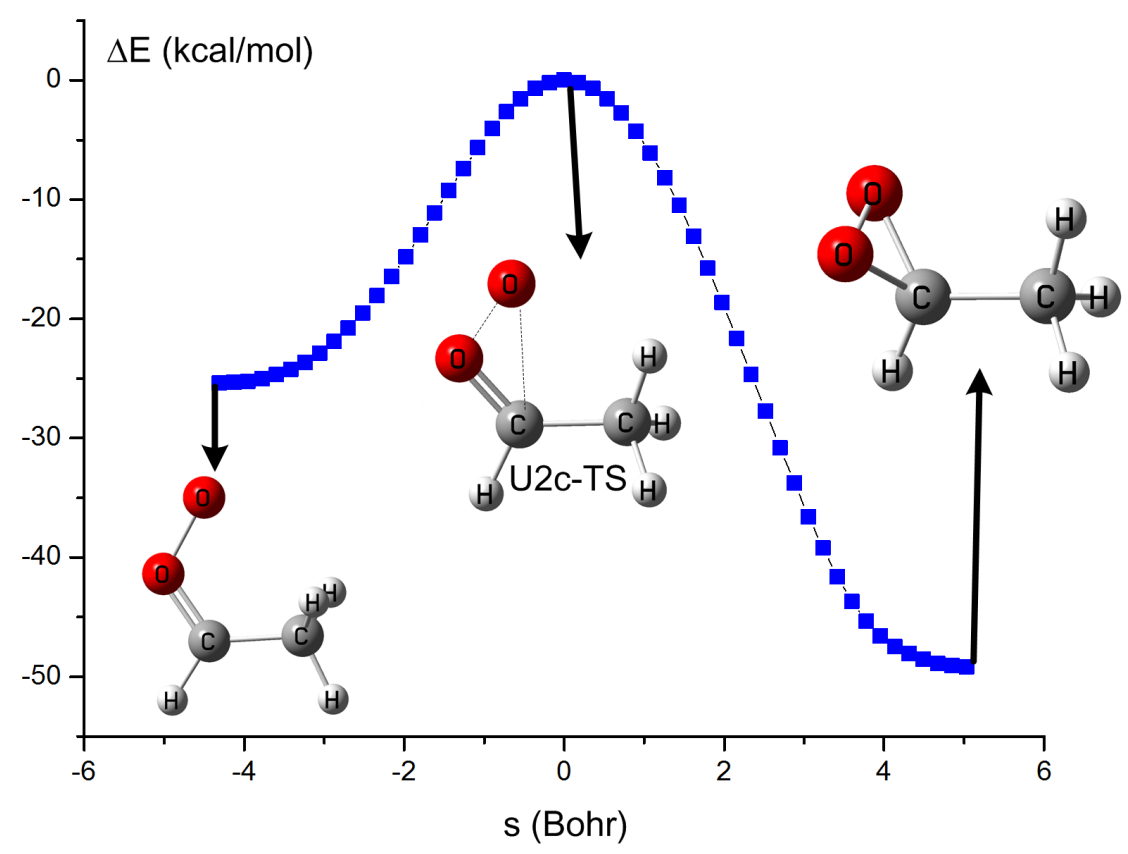

Figure S11. Potential energy profile along minimum energy path through U2c-TS calculated by MN15-L/MG3S.

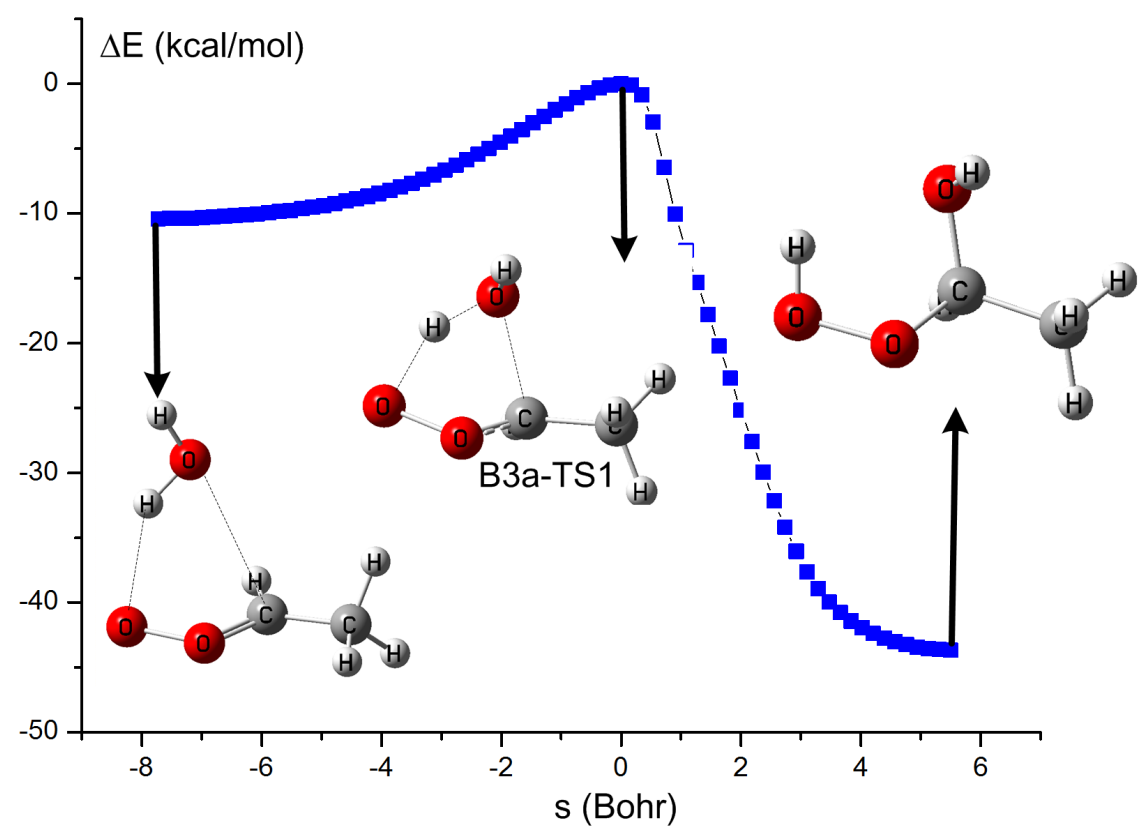

Figure S12. Potential energy profile along minimum energy path through B3a-TS1 calculated by MN15-L/MG3S. 


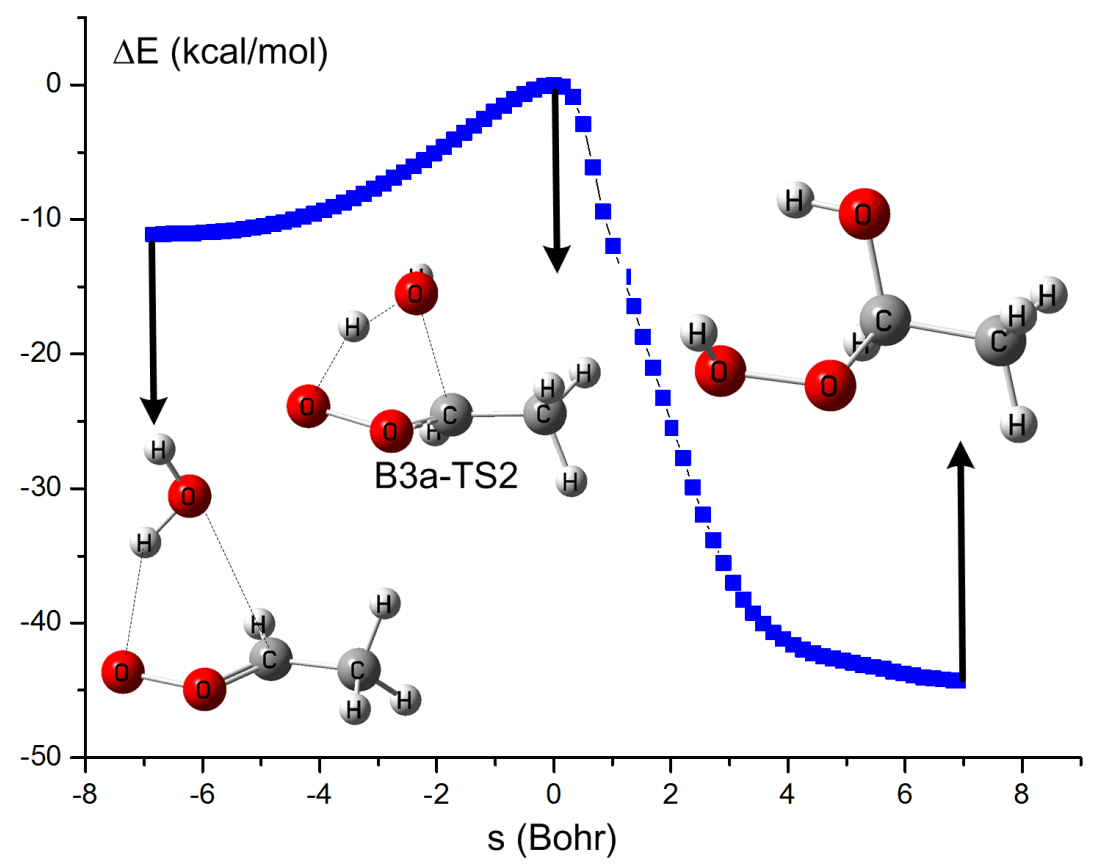

Figure S13. Potential energy profile along minimum energy path through B3a-TS2 calculated by MN15-L/MG3S.

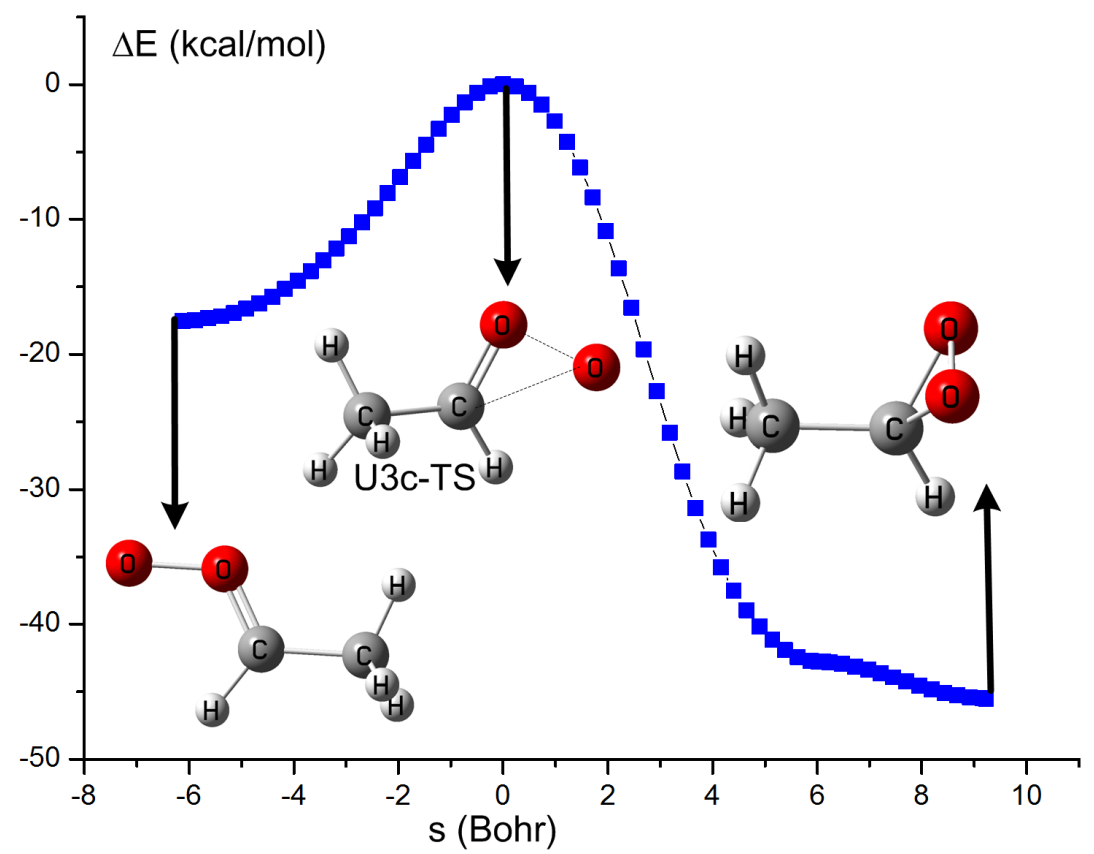

Figure S14. Potential energy profile along minimum energy path through U3C-TS calculated by MN15-L/MG3S. 


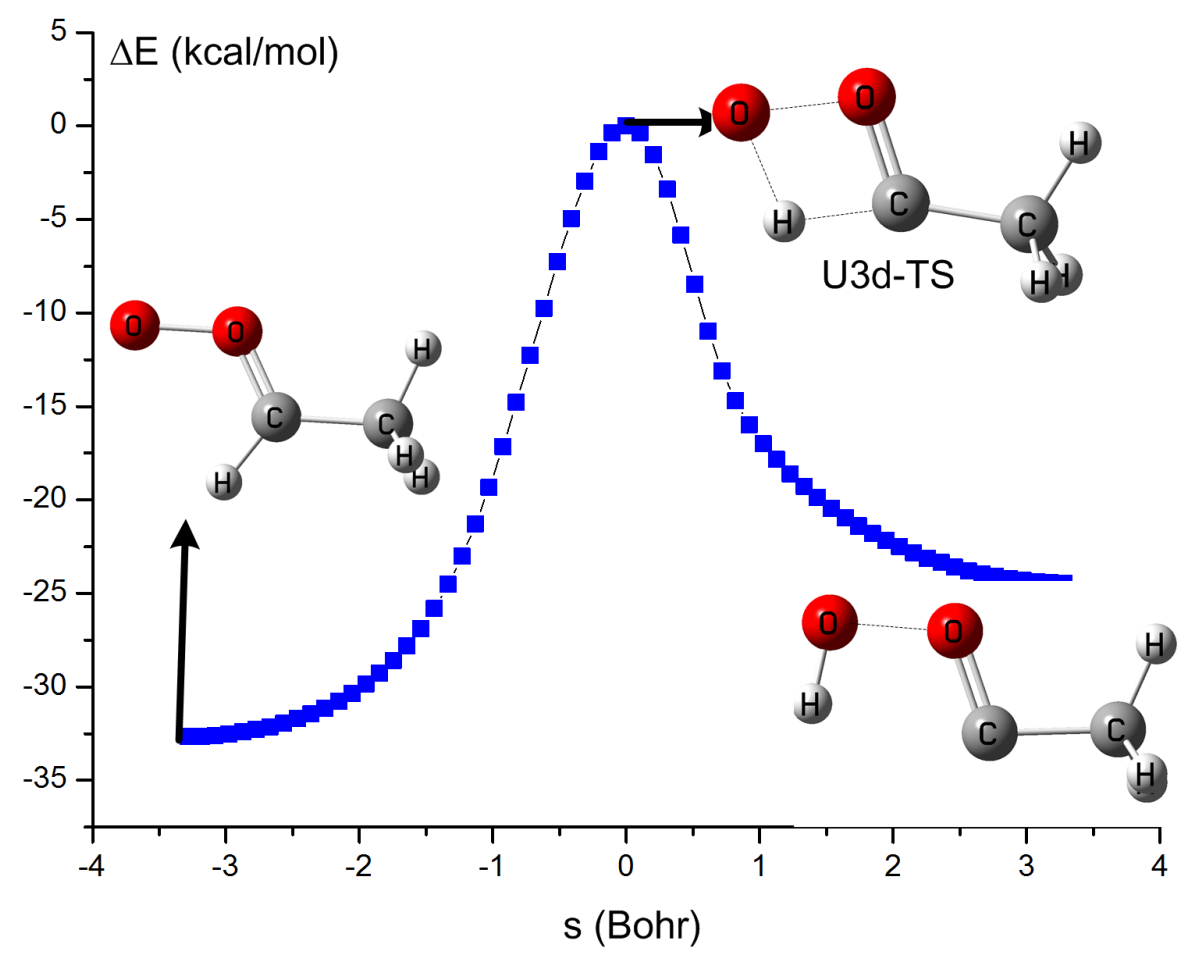

Figure S15. Potential energy profile along minimum energy path through U3D-TS calculated by MN15-L/MG3S. 


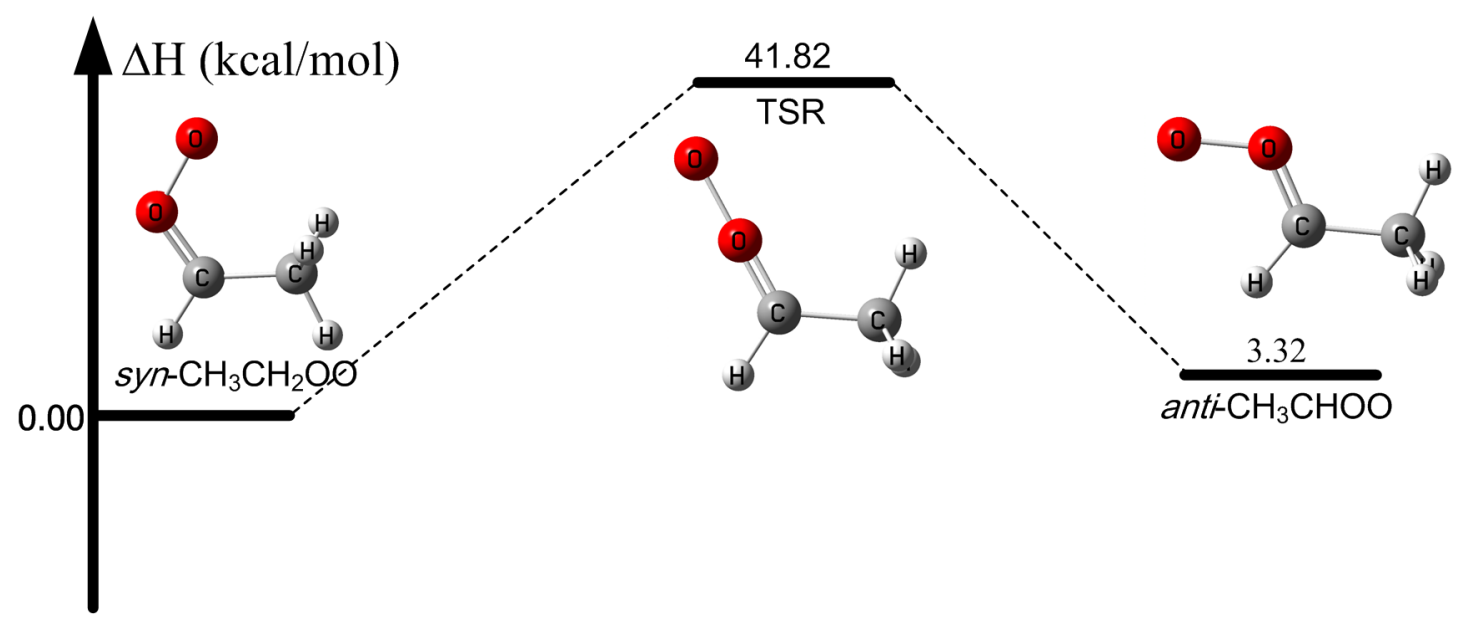

Figure S16. The enthalpy profile at $0 \mathrm{~K}$ for the interconversion of $s y n-\mathrm{CH}_{3} \mathrm{CHOO}$ and anti- $\mathrm{CH}_{3} \mathrm{CHOO}$ as calculated by $\mathrm{W} 3 \mathrm{X}-\mathrm{L} / / \mathrm{QCISD} / \mathrm{TZ}$.

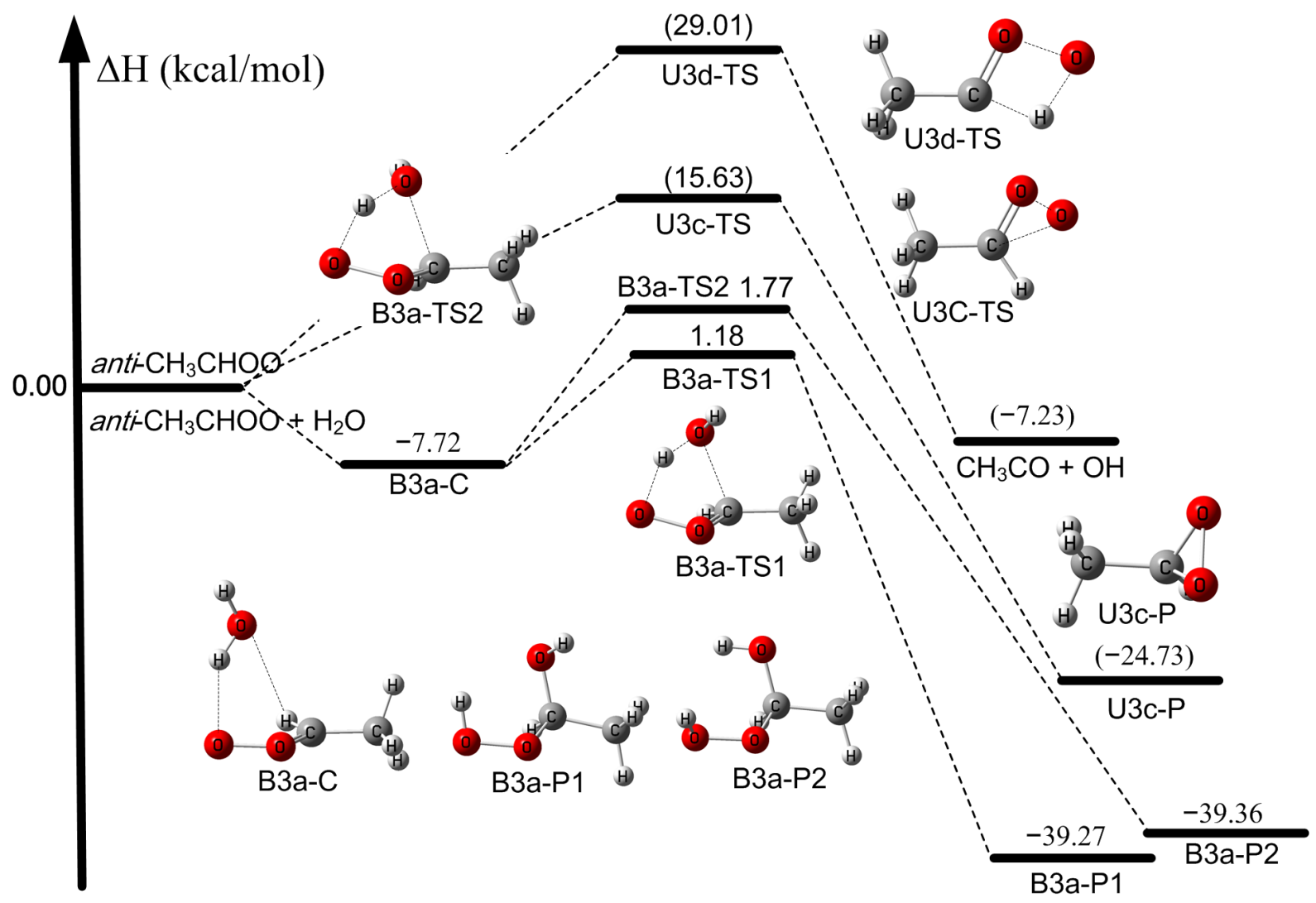

Figure S17. The enthalpy profile of the anti- $\mathrm{CH}_{3} \mathrm{CHOO}+\mathrm{H}_{2} \mathrm{O}$ reaction and its unimolecular reaction at $0 \mathrm{~K}$ as calculated by $\mathrm{W} 3 \mathrm{X}-\mathrm{L} / / \mathrm{QCISD} / \mathrm{TZ}$ and $\mathrm{W} 3 \mathrm{X}-\mathrm{L} / / \mathrm{CCSD}(\mathrm{T})-\mathrm{F} 12 \mathrm{a} / \mathrm{DZ}-\mathrm{F} 12$ (in parentheses). 


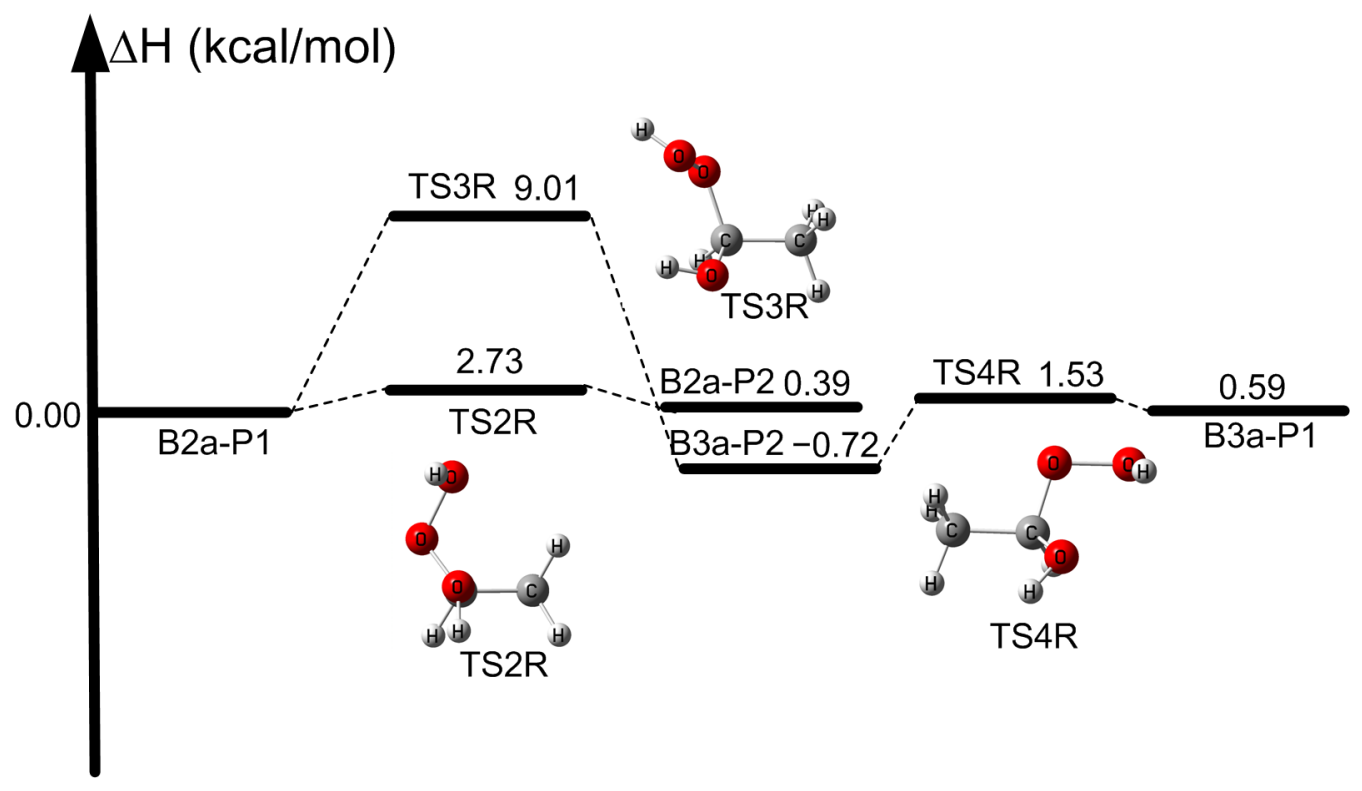

Figure S18. The enthalpy profile of the conversion of intermediates P2A. P2B, P3A, and P3B at $0 \mathrm{~K}$ as calculated by MN15-L/muag-TZ. 


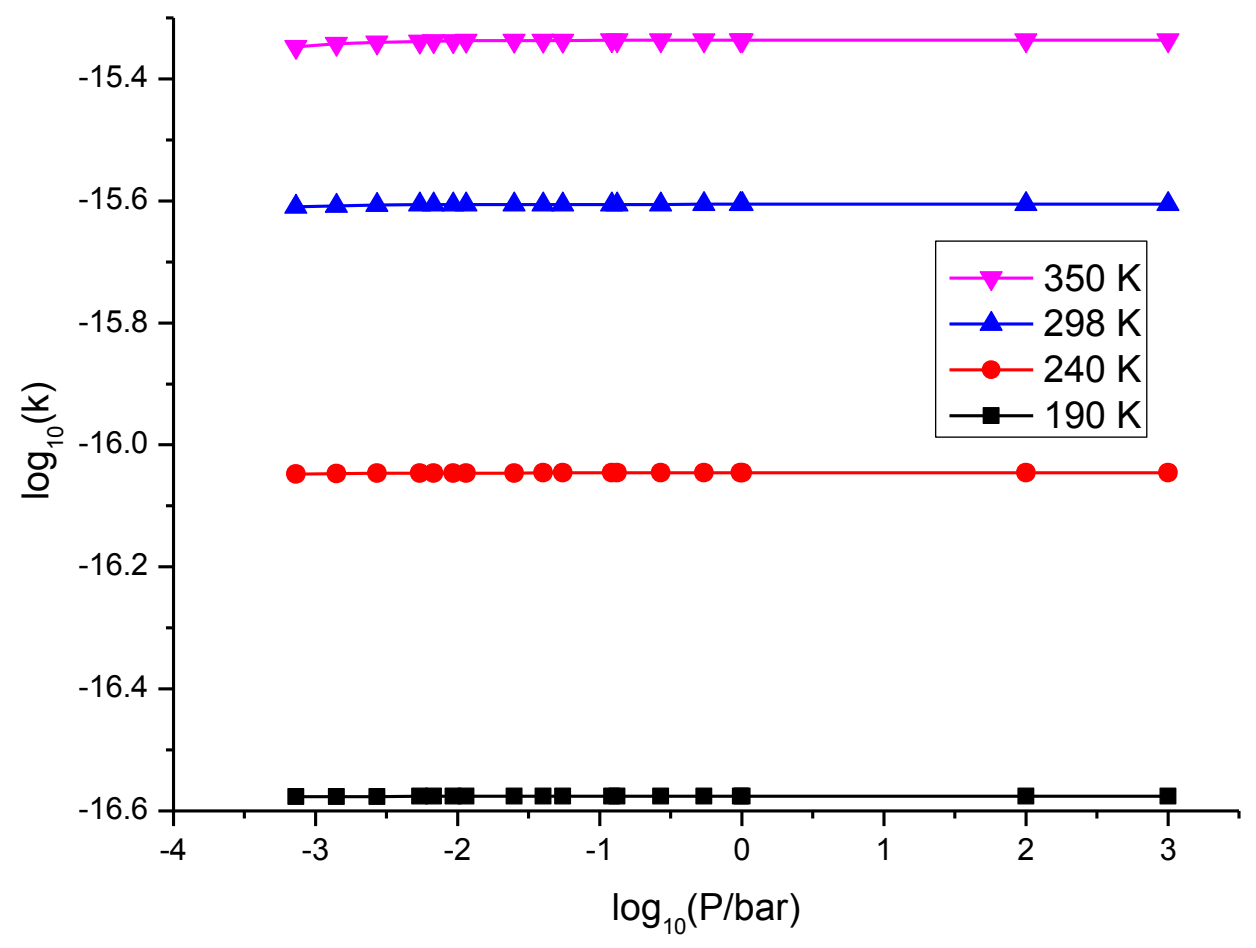

Figure S19. The rate constants of the $\mathrm{CH}_{2} \mathrm{OO}+\mathrm{H}_{2} \mathrm{O}$ reaction (B1a) at different temperature and pressure. The logarithm of the pressure in troposphere is from -0.7 to 0 , and the logarithm of the pressure in stratosphere is from -3.2 to -0.7 . The rate constants at higher pressures are shown to illustrate the high-pressure limit. 


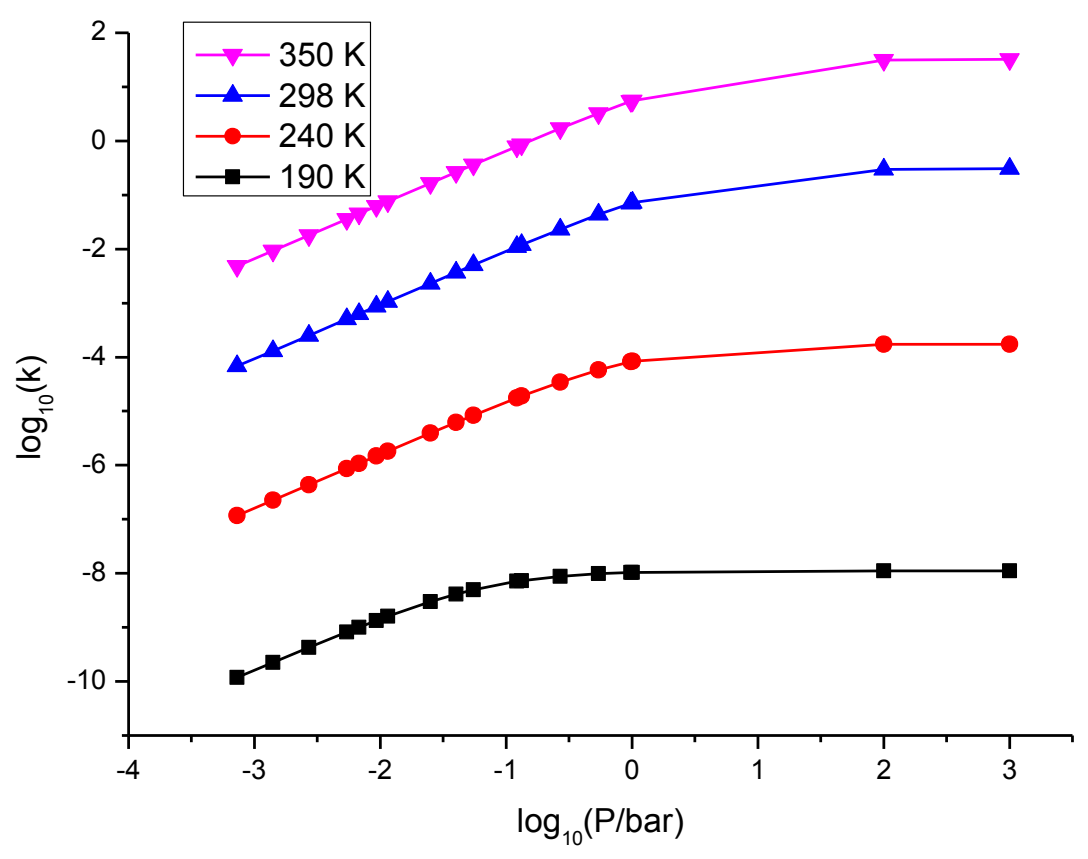

Figure S20. The unimolecular rate constants $\left(\mathrm{s}^{-1}\right)$ of $\mathrm{CH}_{2} \mathrm{OO}$ (U1c) at different temperature and pressure. The logarithm of the pressure in troposphere is from -0.7 to 0 , and the logarithm of the pressure in stratosphere is from -3.2 to -0.7 . The rate constants at higher pressures are shown to illustrate the high-pressure limit. 


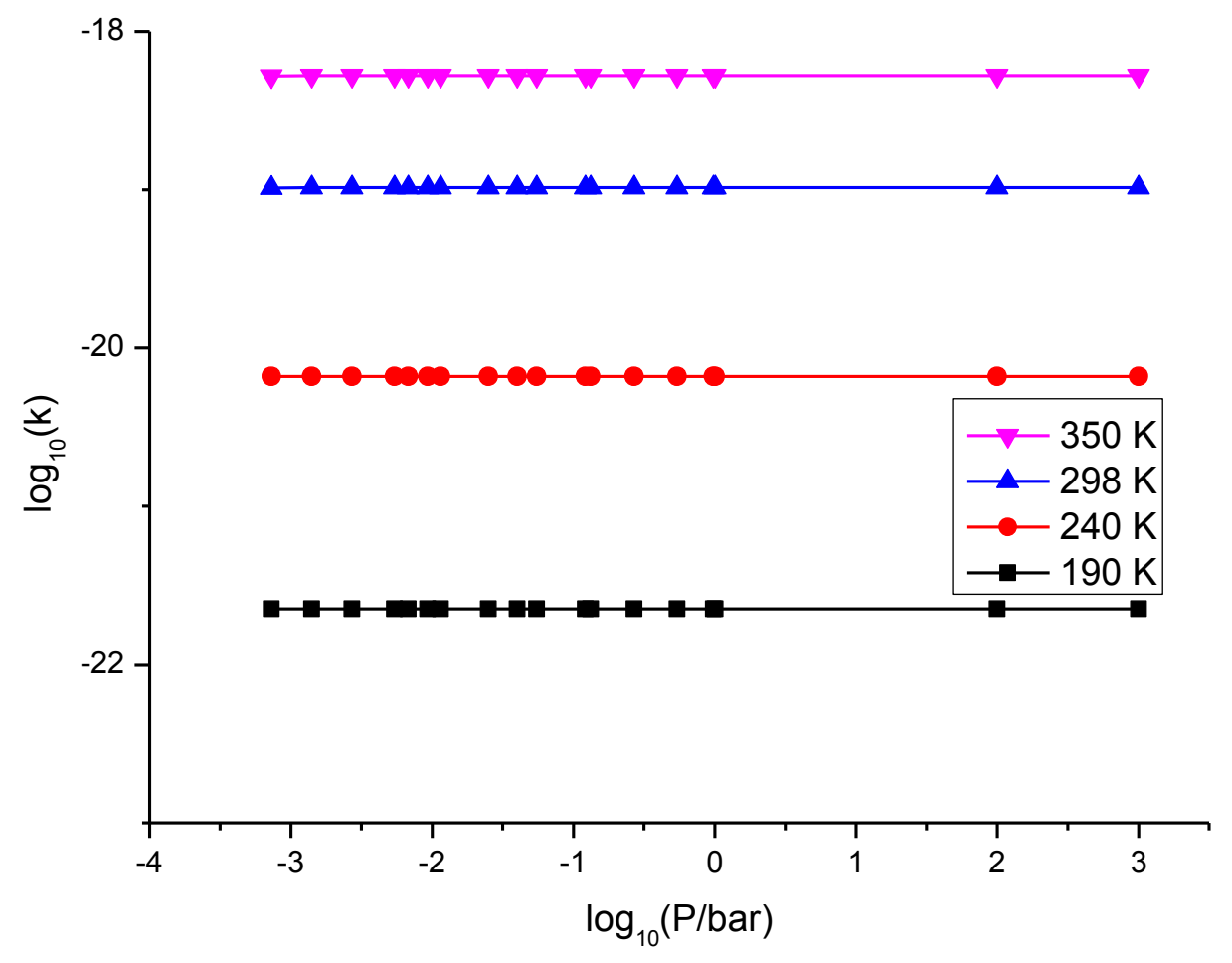

Figure S21. The rate constants of the syn $-\mathrm{CH}_{3} \mathrm{CHOO}+\mathrm{H}_{2} \mathrm{O}$ reaction $(\mathrm{B} 2 \mathrm{a})$ at different temperature and pressure. The logarithm of the pressure in troposphere is from -0.7 to 0 , and the logarithm of the pressure in stratosphere is from -3.2 to -0.7 . The rate constants at higher pressures are shown to illustrate the high-pressure limit. 


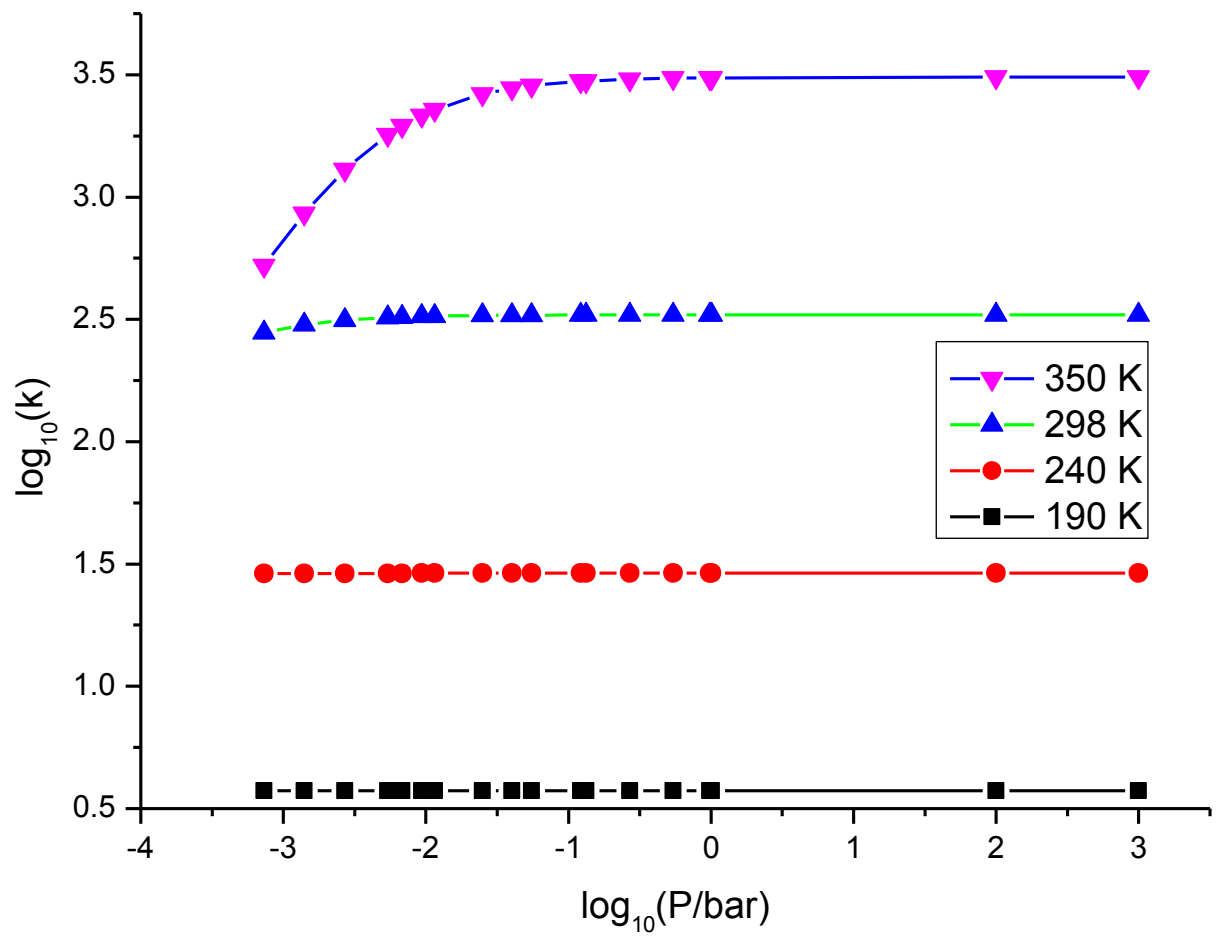

Figure S22. The unimolecular rate constants $\left(\mathrm{s}^{-1}\right)$ of $s y n-\mathrm{CH}_{3} \mathrm{CHOO}$ (U2hs) at different temperature and pressure. The logarithm of the pressure in troposphere is from -0.7 to 0 , and the logarithm of the pressure in stratosphere is from -3.2 to -0.7 . The rate constants at higher pressures are shown to illustrate the high-pressure limit. 


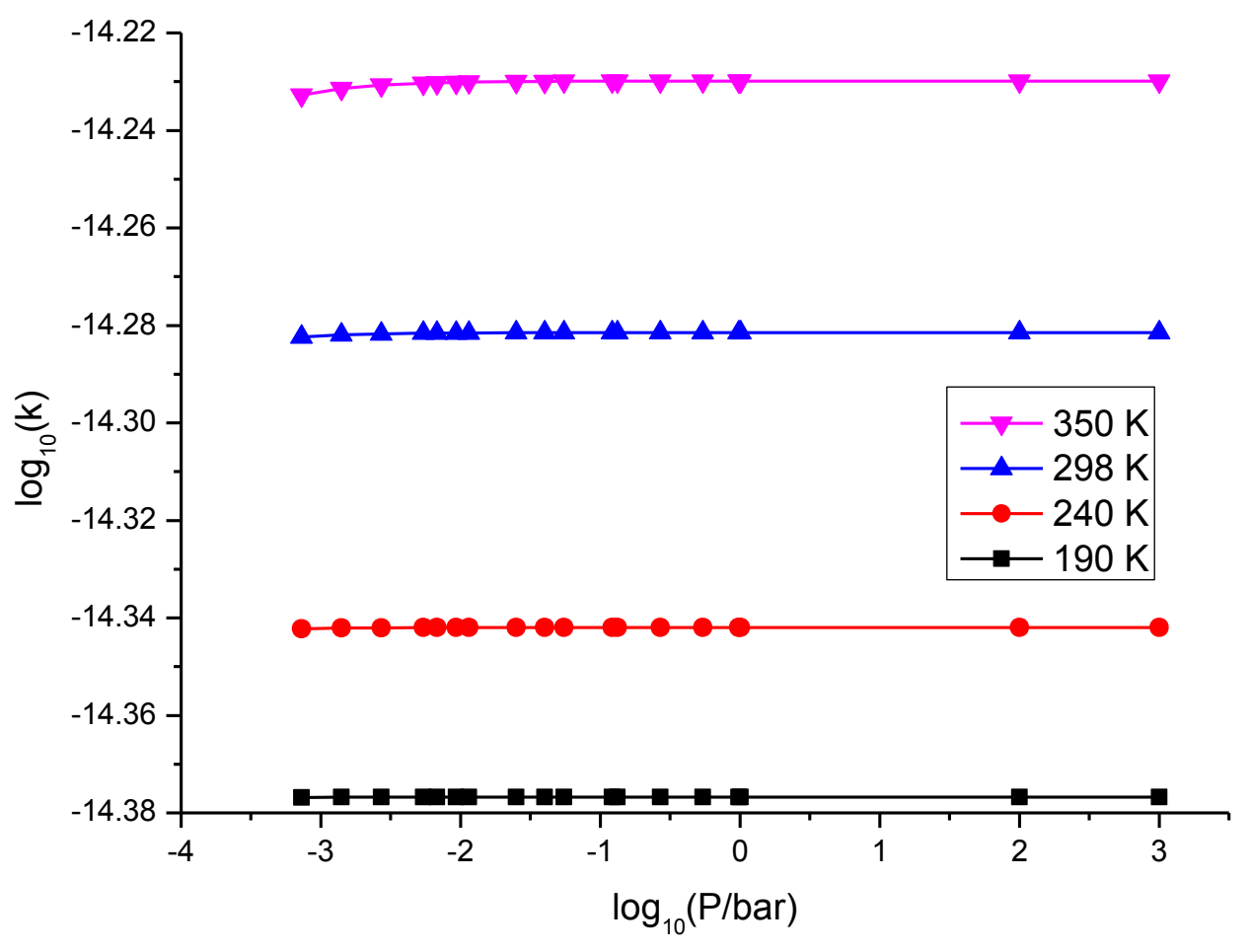

Figure S23. The rate constants of the anti- $\mathrm{CH}_{3} \mathrm{CHOO}+\mathrm{H}_{2} \mathrm{O}$ reaction (B3a) at different temperature and pressure. The logarithm of the pressure in troposphere is from -0.7 to 0 , and the logarithm of the pressure in stratosphere is from -3.2 to -0.7 . The rate constants at higher pressures are shown to illustrate the high-pressure limit. 


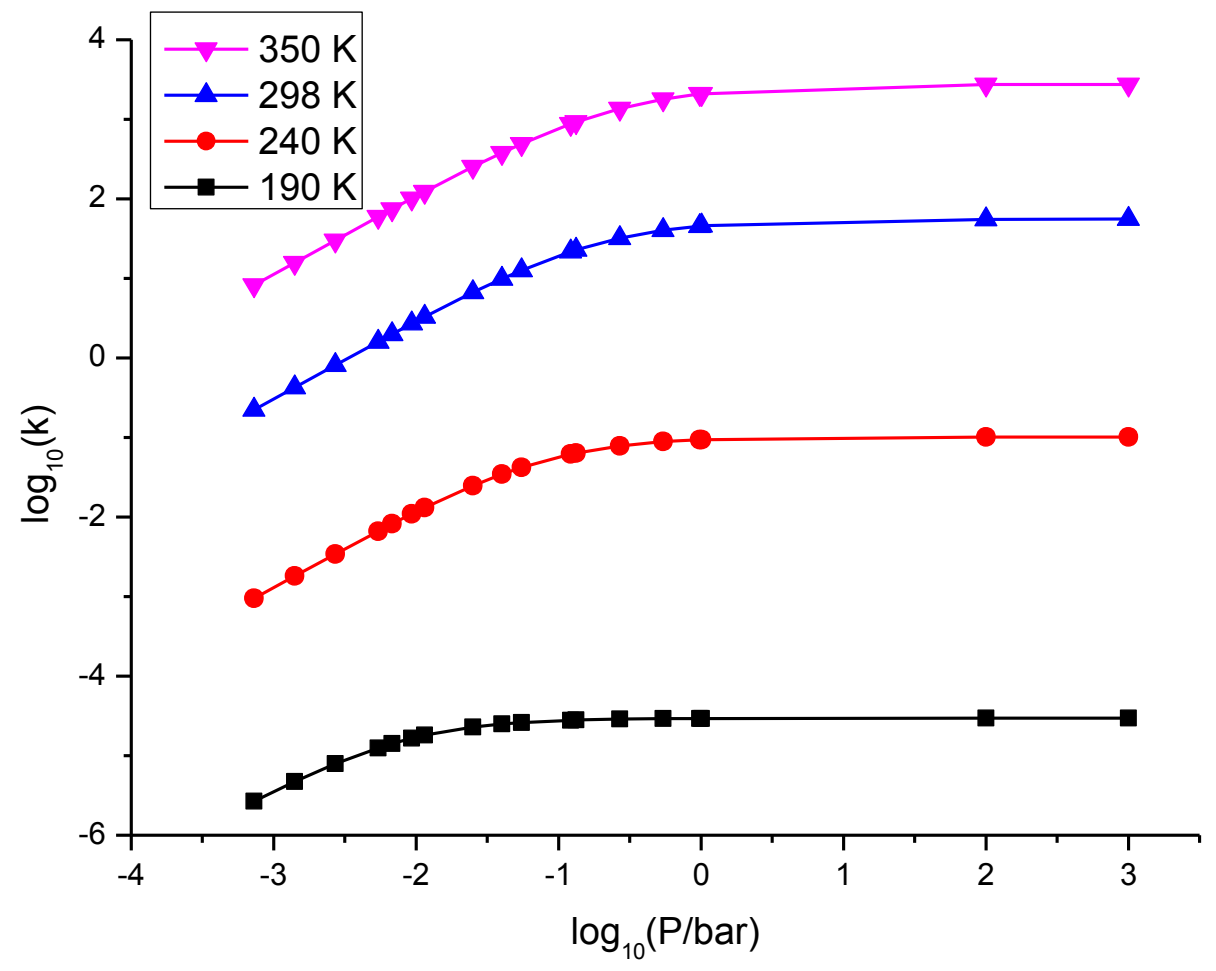

Figure S24. The unimolecular rate constants $\left(\mathrm{s}^{-1}\right)$ of anti- $\mathrm{CH}_{3} \mathrm{CHOO}$ (U3c) at different temperature and pressure. The logarithm of the pressure in troposphere is from -0.7 to 0 , and the logarithm of the pressure in stratosphere is from -3.2 to -0.7 . The rate constants at higher pressures are shown to illustrate the high-pressure limit. 